\title{
Strong convergence for solving a general system of variational inequalities and fixed point problems in Banach spaces
}

\author{
Lu-Chuan Ceng ${ }^{1}$, Nawab Hussain², Abdul Latif ${ }^{2 *}$ and Jen-Chih Yao ${ }^{2,3}$
}

"Correspondence: alatif@kau.edu.sa 2 Department of Mathematics, King Abdulaziz University, P.O. Box 80203, Jeddah, 21589, Saudi Arabia Full list of author information is available at the end of the article

\begin{abstract}
In this paper, we propose and analyze some iterative algorithms by hybrid viscosity approximation methods for solving a general system of variational inequalities and a common fixed point problem of an infinite family of nonexpansive mappings in a uniformly convex Banach space which has a uniformly Gâteaux differentiable norm, and we prove some strong convergence theorems under appropriate conditions. The results presented in this paper improve, extend, supplement and develop the corresponding results recently obtained in the literature.
\end{abstract}

MSC: 49J30; 47H09; 47J20

Keywords: general system of variational inequalities; hybrid viscosity approximation method; nonexpansive mapping; sunny nonexpansive retraction; fixed point; uniformly Gâteaux differentiable norm; uniform convexity

\section{Introduction}

Let $X$ be a real Banach space whose dual space is denoted by $X^{*}$. Let $U=\{x \in X:\|x\|=1\}$ denote the unit sphere of $X$. The Banach space $X$ is said to be uniformly convex if for each $\epsilon \in(0,2]$ there exists $\delta>0$ such that for all $x, y \in U$,

$$
\|x-y\| \geq \epsilon \quad \Rightarrow \quad\|x+y\| / 2 \leq 1-\delta .
$$

It is known that a uniformly convex Banach space is reflexive and strictly convex. The Banach space $X$ is said to be smooth if the limit

$$
\lim _{t \rightarrow 0} \frac{\|x+t y\|-\|x\|}{t}
$$

exists for all $x, y \in U$; in this case, $X$ is also said to have a Gâteaux differentiable norm. $X$ is said to have a uniformly Gâteaux differentiable norm if for each $y \in U$, the limit is attained uniformly for $x \in U$. Moreover, it is said to be uniformly smooth if this limit is attained uniformly for $x, y \in U$. The norm of $X$ is said to be the Fréchet differential if for each $x \in U$, this limit is attained uniformly for $y \in U$. In addition, we define a function $\rho:[0, \infty) \rightarrow[0, \infty)$, called the modulus of smoothness of $X$, as follows:

$$
\rho(\tau)=\sup \left\{\frac{1}{2}(\|x+y\|+\|x-y\|)-1: x, y \in X,\|x\|=1,\|y\|=\tau\right\} .
$$

@ 2013 Ceng et al.; licensee Springer. This is an Open Access article distributed under the terms of the Creative Commons Attribution License (http://creativecommons.org/licenses/by/2.0), which permits unrestricted use, distribution, and reproduction in any medium, provided the original work is properly cited. 
It is known that $X$ is uniformly smooth if and only if $\lim _{\tau \rightarrow 0} \rho(\tau) / \tau=0$. Let $q$ be a fixed real number with $1<q \leq 2$. Then the Banach space $X$ is said to be $q$-uniformly smooth if there exists a constant $c>0$ such that $\rho(\tau) \leq c \tau^{q}$ for all $\tau>0$. As pointed out in [1], no Banach space is $q$-uniformly smooth for $q>2$.

Let $X^{*}$ be the dual of $X$. The normalized duality mapping $J: X \rightarrow 2^{X^{*}}$ is defined by

$$
J(x)=\left\{x^{*} \in X^{*}:\left\langle x, x^{*}\right\rangle=\|x\|^{2}=\left\|x^{*}\right\|^{2}\right\}, \quad \forall x \in X,
$$

where $\langle\cdot, \cdot\rangle$ denotes the generalized duality pairing. It is an immediate consequence of the Hahn-Banach theorem that $J(x)$ is nonempty for each $x \in X$. Moreover, it is known that $J$ is single-valued if and only if $X$ is smooth, whereas if $X$ is uniformly smooth, then the mapping $J$ is norm-to-norm uniformly continuous on bounded subsets of $X$. If $X$ has a uniformly Gateaux differentiable norm, then the duality mapping $J$ is norm-to-weak* uniformly continuous on bounded subsets of $X$.

Let $C$ be a nonempty closed convex subset of a real Banach space $X$. A mapping $T: C \rightarrow$ $C$ is called nonexpansive if

$$
\|T x-T y\| \leq\|x-y\|, \quad \forall x, y \in C .
$$

The set of fixed points of $T$ is denoted by Fix $(T)$. We use the notation $\rightarrow$ to indicate the weak convergence and the one $\rightarrow$ to indicate the strong convergence.

Definition 1.1 Let $A: C \rightarrow X$ be a mapping of $C$ into $X$. Then $A$ is said to be

(i) accretive if for each $x, y \in C$ there exists $j(x-y) \in J(x-y)$ such that

$$
\langle A x-A y, j(x-y)\rangle \geq 0
$$

where $J$ is the normalized duality mapping;

(ii) $\alpha$-strongly accretive if for each $x, y \in C$ there exists $j(x-y) \in J(x-y)$ such that

$$
\langle A x-A y, j(x-y)\rangle \geq \alpha\|x-y\|^{2}
$$

for some $\alpha \in(0,1)$;

(iii) $\beta$-inverse-strongly-accretive if for each $x, y \in C$ there exists $j(x-y) \in J(x-y)$ such that

$$
\langle A x-A y, j(x-y)| \geq \beta\|A x-A y\|^{2},
$$

for some $\beta>0$;

(iv) $\lambda$-strictly pseudocontractive [2] (see also [3]) if for each $x, y \in C$ there exists $j(x-y) \in J(x-y)$ such that

$$
\langle A x-A y, j(x-y)\rangle \leq\|x-y\|^{2}-\lambda\|x-y-(A x-A y)\|^{2}
$$

for some $\lambda \in(0,1)$. 
In the literature, much work has been done related to strong convergence for solving variational inequalities and fixed point problems, see [1-48]. It is worth emphasizing that the definition of the inverse strongly accretive mapping is based on that of the inverse strongly monotone mapping, which was studied by so many authors; see, e.g., [15, 30, 31].

Very recently, Cai and Bu [35] considered the following general system of variational inequalities (GSVI) in a real smooth Banach space $X$, which involves finding $\left(x^{*}, y^{*}\right) \in$ $C \times C$ such that

$$
\begin{cases}\left\langle\mu_{1} B_{1} y^{*}+x^{*}-y^{*}, J\left(x-x^{*}\right)\right\rangle \geq 0, & \forall x \in C, \\ \left\langle\mu_{2} B_{2} x^{*}+y^{*}-x^{*}, J\left(x-y^{*}\right)\right\rangle \geq 0, & \forall x \in C,\end{cases}
$$

where $C$ is a nonempty, closed and convex subset of $X, B_{1}, B_{2}: C \rightarrow X$ are two nonlinear mappings and $\mu_{1}$ and $\mu_{2}$ are two positive constants. Here the set of solutions of GSVI (1.1) is denoted by $\operatorname{GSVI}\left(C, B_{1}, B_{2}\right)$. In particular, if $X=H$, a real Hilbert space, then GSVI (1.1) reduces to the following GSVI of finding $\left(x^{*}, y^{*}\right) \in C \times C$ such that

$$
\begin{cases}\left\langle\mu_{1} B_{1} y^{*}+x^{*}-y^{*}, x-x^{*}\right\rangle \geq 0, & \forall x \in C, \\ \left\langle\mu_{2} B_{2} x^{*}+y^{*}-x^{*}, x-y^{*}\right\rangle \geq 0, & \forall x \in C,\end{cases}
$$

where $\mu_{1}$ and $\mu_{2}$ are two positive constants. The set of solutions of problem (1.2) is still denoted by $\operatorname{GSVI}\left(C, B_{1}, B_{2}\right)$. In particular, if $B_{1}=B_{2}=A$, then problem (1.2) reduces to the new system of variational inequalities (NSVI), introduced and studied by Verma [5]. Further, if $x^{*}=y^{*}$ additionally, then the NSVI reduces to the classical variational inequality problem (VIP) of finding $x^{*} \in C$ such that

$$
\left\langle A x^{*}, x-x^{*}\right\rangle \geq 0, \quad \forall x \in C
$$

The solution set of VIP $(1.3)$ is denoted by $\operatorname{VI}(C, A)$. Variational inequality theory has been studied quite extensively and has emerged as an important tool in the study of a wide class of obstacle, unilateral, free, moving, equilibrium problems. It is now well known that the variational inequalities are equivalent to the fixed point problems, the origin of which can be traced back to Lions and Stampacchia [13]. This alternative formulation has been used to suggest and analyze the projection iterative method for solving variational inequalities under the conditions that the involved operator must be strongly monotone and Lipschitz continuous.

Recently, Ceng, Wang and Yao [4] transformed problem (1.2) into a fixed point problem in the following way.

Lemma 1.1 (see [4]) For given $\bar{x}, \bar{y} \in C,(\bar{x}, \bar{y})$ is a solution of problem (1.1) if and only if $\bar{x}$ is a fixed point of the mapping $G: C \rightarrow C$ defined by

$$
G(x)=P_{C}\left[P_{C}\left(x-\mu_{2} B_{2} x\right)-\mu_{1} B_{1} P_{C}\left(x-\mu_{2} B_{2} x\right)\right], \quad \forall x \in C,
$$

where $\bar{y}=P_{C}\left(\bar{x}-\mu_{2} B_{2} \bar{x}\right)$.

In particular, if the mappings $B_{i}: C \rightarrow H$ are $\beta_{i}$-inverse strongly monotone for $i=1,2$, then the mapping $G$ is nonexpansive provided $\mu_{i} \in\left(0,2 \beta_{i}\right)$ for $i=1,2$. 
In 1976, Korpelevich [6] proposed an iterative algorithm for solving VIP (1.3) in the Euclidean space $\mathbf{R}^{n}$ :

$$
\left\{\begin{array}{l}
y_{n}=P_{C}\left(x_{n}-\tau A x_{n}\right), \\
x_{n+1}=P_{C}\left(x_{n}-\tau A y_{n}\right), \quad n \geq 0,
\end{array}\right.
$$

with $\tau>0$ a given number, which is known as the extragradient method (see also [7, 8]). The literature on the VIP is vast and Korpelevich's extragradient method has received great attention given by many authors, who improved it in various ways; see, e.g., [9-27, $35,40]$ and references therein, to name but a few.

In particular, whenever $X$ is still a real smooth Banach space, $B_{1}=B_{2}=A$ and $x^{*}=y^{*}$, then GSVI (1.1) reduces to the variational inequality problem (VIP) of finding $x^{*} \in C$ such that

$$
\left\langle A x^{*}, J\left(x-x^{*}\right)\right| \geq 0, \quad \forall x \in C
$$

which was considered by Aoyama, Iiduka and Takahashi [28]. Note that VIP (1.5) is connected with the fixed point problem for a nonlinear mapping (see, e.g., [41]), the problem of finding a zero point of a nonlinear operator (see, e.g., [36]) and so on. It is clear that VIP (1.5) extends VIP (1.3) from Hilbert spaces to Banach spaces.

In order to find a solution of VIP (1.5), Aoyama, liduka and Takahashi [28] introduced the following iterative scheme for an accretive operator $A$ :

$$
x_{n+1}=\alpha_{n} x_{n}+\left(1-\alpha_{n}\right) \Pi_{C}\left(x_{n}-\lambda_{n} A x_{n}\right), \quad \forall n \geq 1,
$$

where $\Pi_{C}$ is a sunny nonexpansive retraction from $X$ onto $C$. Then they proved a weak convergence theorem. For related work, see [29] and the references therein.

Recently, Jung [43] introduced and analyzed a composite iterative algorithm by the viscosity approximation method for solving a fixed point problem of a nonexpansive mapping and VIP (1.3) for an inverse-strongly monotone mapping $A$ in a real Hilbert space $H$.

Theorem 1.1 (see [43, Theorem 3.1]) Let $C$ be a nonempty closed convex subset of a real Hilbert space $H$. Let $A: C \rightarrow H$ be an $\alpha$-inverse-strongly monotone mapping, let $S: C \rightarrow$ $C$ be a nonexpansive mapping such that $\operatorname{Fix}(S) \cap \operatorname{VI}(C, A) \neq \emptyset$, and let $f: C \rightarrow C$ be a contraction with coefficient $\rho \in(0,1)$. Let $\left\{x_{n}\right\}$ be the sequence generated by

$$
\left\{\begin{array}{l}
x_{0}=x \in C \quad \text { chosen arbitrarily, } \\
y_{n}=\alpha_{n} f\left(x_{n}\right)+\left(1-\alpha_{n}\right) S P_{C}\left(x_{n}-\lambda_{n} A x_{n}\right), \\
x_{n+1}=\left(1-\beta_{n}\right) y_{n}+\beta_{n} S P_{C}\left(y_{n}-\lambda_{n} A y_{n}\right), \quad \forall n \geq 0,
\end{array}\right.
$$

where $\left\{\lambda_{n}\right\} \subset[0,2 \alpha],\left\{\alpha_{n}\right\} \subset[0,1)$ and $\left\{\beta_{n}\right\} \subset[0,1]$. If $\left\{\alpha_{n}\right\},\left\{\lambda_{n}\right\}$ and $\left\{\beta_{n}\right\}$ satisfy the following conditions:

(i) $\lim _{n \rightarrow \infty} \alpha_{n}=0$ and $\sum_{n=0}^{\infty} \alpha_{n}=\infty$,

(ii) $\left\{\beta_{n}\right\} \subset[0, a), \forall n \geq 0$ for some $a \in(0,1)$,

(iii) $\left\{\lambda_{n}\right\} \subset[c, d]$ for some $c, d \in(0,2 \alpha)$,

(iv) $\sum_{n=0}^{\infty}\left|\alpha_{n+1}-\alpha_{n}\right|<\infty, \sum_{n=0}^{\infty}\left|\beta_{n+1}-\beta_{n}\right|<\infty$ and $\sum_{n=0}^{\infty}\left|\lambda_{n+1}-\lambda_{n}\right|<\infty$, 
then $\left\{x_{n}\right\}$ converges strongly to $q \in \operatorname{Fix}(S) \cap \operatorname{VI}(C, A)$, which solves the VIP

$$
\langle q-f(q), q-p\rangle \leq 0, \quad \forall p \in \operatorname{Fix}(S) \cap \operatorname{VI}(C, A) .
$$

Beyond doubt, it is an interesting and valuable problem of how to construct some algorithms with strong convergence for solving GSVI (1.1) which contains VIP (1.5) as a special case. Very recently, Cai and $\mathrm{Bu}$ [35] constructed an iterative algorithm for solving GSVI (1.1) and a common fixed point problem of an infinite family of nonexpansive mappings in a uniformly convex and 2-uniformly smooth Banach space. They proved strong convergence of the proposed method by virtue of the following inequality in a 2-uniformly smooth Banach space $X$.

Lemma 1.2 (see [32]) Let X be a 2-uniformly smooth Banach space. Then

$$
\|x+y\|^{2} \leq\|x\|^{2}+2\langle y, J(x)\rangle+2\|\kappa y\|^{2}, \quad \forall x, y \in X
$$

where $\kappa$ is the 2-uniformly smooth constant of $X$ and $J$ is the normalized duality mapping from $X$ into $X^{*}$.

Define the mapping $G: C \rightarrow C$ as follows:

$$
G(x):=\Pi_{C}\left(I-\mu_{1} B_{1}\right) \Pi_{C}\left(I-\mu_{2} B_{2}\right) x, \quad \forall x \in C .
$$

The fixed point set of $G$ is denoted by $\Omega$. Then their strong convergence theorem on the proposed method is stated as follows.

Theorem 1.2 (see [35, Theorem 3.1]) Let C be a nonempty closed convex subset of a uniformly convex and 2-uniformly smooth Banach space $X$. Let $\Pi_{C}$ be a sunny nonexpansive retraction from $X$ onto $C$. Let the mapping $B_{i}: C \rightarrow X$ be $\alpha_{i}$-inverse-strongly accretive with $0<\mu_{i}<\frac{\alpha_{i}}{\kappa^{2}}$ for $i=1,2$. Let $f$ be a contraction of $C$ into itself with coefficient $\delta \in(0,1)$. Let $\left\{S_{n}\right\}_{n=1}^{\infty}$ be an infinite family of nonexpansive mappings of $C$ into itself such that $F=\bigcap_{i=1}^{\infty} \operatorname{Fix}\left(S_{i}\right) \cap \Omega \neq \emptyset$, where $\Omega$ is the fixed point set of the mapping $G$ defined by (1.7). For arbitrarily given $x_{1} \in C$, let $\left\{x_{n}\right\}$ be the sequence generated by

$$
\left\{\begin{array}{l}
x_{n+1}=\beta_{n} x_{n}+\left(1-\beta_{n}\right) S_{n} y_{n}, \\
y_{n}=\alpha_{n} f\left(x_{n}\right)+\left(1-\alpha_{n}\right) z_{n}, \\
z_{n}=\Pi_{C}\left(u_{n}-\mu_{1} B_{1} u_{n}\right), \\
u_{n}=\Pi_{C}\left(x_{n}-\mu_{2} B_{2} x_{n}\right), \quad \forall n \geq 1 .
\end{array}\right.
$$

Suppose that $\left\{\alpha_{n}\right\}$ and $\left\{\beta_{n}\right\}$ are two sequences in $(0,1)$ satisfying the following conditions:

(i) $0<\liminf _{n \rightarrow \infty} \beta_{n} \leq \lim \sup _{n \rightarrow \infty} \beta_{n}<1$;

(ii) $\lim _{n \rightarrow \infty} \alpha_{n}=0$ and $\sum_{n=1}^{\infty} \alpha_{n}=\infty$.

Assume that $\sum_{n=1}^{\infty} \sup _{x \in D}\left\|S_{n+1} x-S_{n} x\right\|<\infty$ for any bounded subset $D$ of $C$, and let $S$ be a mapping of $C$ into $X$ defined by $S x=\lim _{n \rightarrow \infty} S_{n} x$ for all $x \in C$ and suppose that $\operatorname{Fix}(S)=$ $\bigcap_{n=1}^{\infty} \operatorname{Fix}\left(S_{n}\right)$. Then $\left\{x_{n}\right\}$ converges strongly to $q \in F$, which solves the following VIP:

$$
\langle q-f(q), J(q-p)\rangle \leq 0, \quad \forall p \in F .
$$


We remark that in Theorem 1.2, the Banach space $X$ is both uniformly convex and 2-uniformly smooth. According to Lemma 1.2, the 2-uniform smoothness of $X$ guarantees the nonexpansivity of the mapping $I-\mu_{i} B_{i}$ for an $\alpha_{i}$-inverse-strongly accretive mapping $B_{i}: C \rightarrow X$ with $0 \leq \mu_{i} \leq \frac{\alpha_{i}}{\kappa^{2}}$ for $i=1,2$, and hence the composite mapping $G: C \rightarrow C$ is nonexpansive where $G=\Pi_{C}\left(I-\mu_{1} B_{1}\right) \Pi_{C}\left(I-\mu_{2} B_{2}\right)$. In the meantime, for the convenience of implementing the argument techniques in [4], the assumption of real smoothness and uniform convexity on $X$ guarantees that the following inequality holds (see [42]): for any given $r>0$, there exists a strictly increasing, continuous and convex function $g:[0,2 r] \rightarrow \mathbf{R}, g(0)=0$ such that

$$
g(\|x-y\|) \leq\|x\|^{2}-2\langle x, J(y)\rangle+\|y\|^{2}, \quad \forall x, y \in B_{r},
$$

where $B_{r}=\{x \in X:\|x\| \leq r\}$.

Naturally, we wonder whether the uniform convexity and 2-uniform smoothness of $X$ can be replaced by the weaker geometrical property of $X$ or not. There is no doubt that it is an interesting problem worth investigating.

In this paper, motivated by the above facts, we introduce and study two implicit iterative algorithms and two explicit iterative algorithms by hybrid viscosity approximation methods for finding a common element of the set of solutions of GSVI (1.1) and the set of common fixed points of an infinite family of nonexpansive mappings in a real uniformly convex Banach space which has a uniformly Gâteaux differentiable norm. We prove some strong convergence theorems under appropriate conditions. Our results improve, extend, supplement and develop recent corresponding results in the literature, especially [35, Theorem 3.1] in the following aspects. First, the assumption of the uniformly convex and 2-uniformly smooth Banach space $X$ in [35, Theorem 3.1] is weakened to the one of the uniformly convex Banach space $X$ having a uniformly Gâteaux differentiable norm in our results. Second, the proof in [35, Theorem 3.1] depends on the argument techniques in [4], inequality (1.6) in 2-uniformly smooth Banach spaces and inequality (1.9) in smooth and uniform convex Banach spaces. It is worth emphasizing that the proof in our results depends on no argument techniques in [4] but use the inequality in uniform convex Banach spaces; see Lemma 2.5 in Section 2 of this paper. Third, the four iterative algorithms proposed in this paper are very different from the iterative algorithm in [35, Theorem 3.1] because two iterative algorithms are implicit ones and the iterative step of computing $x_{n+1}$ in other two explicit iterative algorithms involves the sum of three terms.

\section{Preliminaries}

We list some lemmas that will be used in the sequel. Lemma 2.1 can be found in [34]. Lemma 2.2 is an immediate consequence of the subdifferential inequality of the function $\frac{1}{2}\|\cdot\|^{2}$.

Lemma 2.1 Let $\left\{s_{n}\right\}$ be a sequence of nonnegative real numbers satisfying

$$
s_{n+1} \leq\left(1-\alpha_{n}\right) s_{n}+\alpha_{n} \beta_{n}+\gamma_{n}, \quad \forall n \geq 0,
$$

where $\left\{\alpha_{n}\right\},\left\{\beta_{n}\right\}$ and $\left\{\gamma_{n}\right\}$ satisfy the conditions:

(i) $\left\{\alpha_{n}\right\} \subset[0,1]$ and $\sum_{n=0}^{\infty} \alpha_{n}=\infty$; 
(ii) $\lim \sup _{n \rightarrow \infty} \beta_{n} \leq 0$;

(iii) $\gamma_{n} \geq 0, \forall n \geq 0$, and $\sum_{n=0}^{\infty} \gamma_{n}<\infty$.

Then $\lim \sup _{n \rightarrow \infty} s_{n}=0$.

Lemma 2.2 In a smooth Banach space X, the following inequality holds:

$$
\|x+y\|^{2} \leq\|x\|^{2}+2\langle y, J(x+y)\rangle, \quad \forall x, y \in X .
$$

Lemma 2.3 (see [39]) Let $\left\{x_{n}\right\}$ and $\left\{z_{n}\right\}$ be bounded sequences in a Banach space $X$, and let $\left\{\alpha_{n}\right\}$ be a sequence in $[0,1]$ which satisfies the following condition

$$
0<\liminf _{n \rightarrow \infty} \alpha_{n} \leq \limsup _{n \rightarrow \infty} \alpha_{n}<1
$$

Suppose $x_{n+1}=\alpha_{n} x_{n}+\left(1-\alpha_{n}\right) z_{n}, \forall n \geq 0$ and $\lim \sup _{n \rightarrow \infty}\left(\left\|z_{n+1}-z_{n}\right\|-\left\|x_{n+1}-x_{n}\right\|\right) \leq 0$. Then $\lim _{n \rightarrow \infty}\left\|z_{n}-x_{n}\right\|=0$.

Let $D$ be a subset of $C$ and let $\Pi$ be a mapping of $C$ into $D$. Then $\Pi$ is said to be sunny if

$$
\Pi[\Pi(x)+t(x-\Pi(x))]=\Pi(x)
$$

whenever $\Pi(x)+t(x-\Pi(x)) \in C$ for $x \in C$ and $t \geq 0$. A mapping $\Pi$ of $C$ into itself is called a retraction if $\Pi^{2}=\Pi$. If a mapping $\Pi$ of $C$ into itself is a retraction, then $\Pi(z)=z$ for every $z \in R(\Pi)$, where $R(\Pi)$ is the range of $\Pi$. A subset $D$ of $C$ is called a sunny nonexpansive retract of $C$ if there exists a sunny nonexpansive retraction from $C$ onto $D$. The following lemma concerns the sunny nonexpansive retraction.

Lemma 2.4 [33] Let $C$ be a nonempty closed convex subset of a real smooth Banach space $X$. Let $D$ be a nonempty subset of $C$. Let $\Pi$ be a retraction of $C$ onto $D$. Then the following are equivalent:

(i) $\Pi$ is sunny and nonexpansive;

(ii) $\|\Pi(x)-\Pi(y)\|^{2} \leq\langle x-y, J(\Pi(x)-\Pi(y))\rangle, \forall x, y \in C$;

(iii) $\langle x-\Pi(x), J(y-\Pi(x))\rangle \leq 0, \forall x \in C, y \in D$.

It is well known that if $X=H$, a Hilbert space, then a sunny nonexpansive retraction $\Pi_{C}$ is coincident with the metric projection from $X$ onto $C$; that is, $\Pi_{C}=P_{C}$. If $C$ is a nonempty closed convex subset of a strictly convex and uniformly smooth Banach space $X$ and if $T: C \rightarrow C$ is a nonexpansive mapping with the fixed point set $\operatorname{Fix}(T) \neq \emptyset$, then the set $\operatorname{Fix}(T)$ is a sunny nonexpansive retract of $C$.

Lemma 2.5 (see [32]) Given a number $r>0$, a real Banach space $X$ is uniformly convex if and only if there exists a continuous strictly increasing function $g:[0, \infty) \rightarrow[0, \infty)$, $g(0)=0$, such that

$$
\|\lambda x+(1-\lambda) y\|^{2} \leq \lambda\|x\|^{2}+(1-\lambda)\|y\|^{2}-\lambda(1-\lambda) g(\|x-y\|)
$$

for all $\lambda \in[0,1]$ and $x, y \in X$ such that $\|x\| \leq r$ and $\|y\| \leq r$. 
Lemma 2.6 (see [37]) Let $C$ be a nonempty closed convex subset of a Banach space X. Let $S_{0}, S_{1}, \ldots$ be a sequence of mappings of $C$ into itself. Suppose that $\sum_{n=1}^{\infty} \sup \left\{\left\|S_{n} x-S_{n-1} x\right\|\right.$ : $x \in C\}<\infty$. Then, for each $y \in C,\left\{S_{n} y\right\}$ converges strongly to some point of C. Moreover, let $S$ be a mapping of $C$ into itself defined by $S y=\lim _{n \rightarrow \infty} S_{n} y$ for all $y \in C$. Then $\lim _{n \rightarrow \infty} \sup \left\{\left\|S x-S_{n} x\right\|: x \in C\right\}=0$.

Let $C$ be a nonempty closed convex subset of a Banach space $X$, and let $T: C \rightarrow C$ be a nonexpansive mapping with $\operatorname{Fix}(T) \neq \emptyset$. As previously, let $\Xi_{C}$ be the set of all contractions on $C$. For $t \in(0,1)$ and $f \in \Xi_{C}$, let $x_{t} \in C$ be the unique fixed point of the contraction $x \mapsto t f(x)+(1-t) T x$ on $C$; that is,

$$
x_{t}=t f\left(x_{t}\right)+(1-t) T x_{t} .
$$

Lemma 2.7 (see [41]) Let $X$ be a reflexive and strictly convex Banach space with a uniformly Gâteaux differentiable norm. Let $C$ be a nonempty closed convex subset of $X$, let $T: C \rightarrow C$ be a nonexpansive mapping with $\operatorname{Fix}(T) \neq \emptyset$, and $f \in \Xi_{C}$. Then the net $\left\{x_{t}\right\}$ defined by $x_{t}=t f\left(x_{t}\right)+(1-t) T x_{t}$ converges strongly to a point in $\operatorname{Fix}(T)$. If we define a mapping $Q: \Xi_{C} \rightarrow \operatorname{Fix}(T)$ by $Q(f):=s-\lim _{t \rightarrow 0} x_{t}, \forall f \in \Xi_{C}$, then $Q(f)$ solves the VIP

$$
\langle(I-f) Q(f), J(Q(f)-p)\rangle \leq 0, \quad \forall f \in \Xi_{C}, p \in \operatorname{Fix}(T) .
$$

Lemma 2.8 (see [38]) Let $C$ be a nonempty closed convex subset of a strictly convex Banach space X. Let $\left\{T_{n}\right\}_{n=0}^{\infty}$ be a sequence of nonexpansive mappings on C. Suppose that $\bigcap_{n=0}^{\infty} \operatorname{Fix}\left(T_{n}\right)$ is nonempty. Let $\left\{\lambda_{n}\right\}$ be a sequence of positive numbers with $\sum_{n=0}^{\infty} \lambda_{n}=1$. Then a mapping $S$ on $C$ defined by $S x=\sum_{n=0}^{\infty} \lambda_{n} T_{n} x$ for $x \in C$ is well defined, nonexpansive and $\operatorname{Fix}(S)=\bigcap_{n=0}^{\infty} \operatorname{Fix}\left(T_{n}\right)$ holds.

Lemma 2.9 Let $C$ be a nonempty closed convex subset of a smooth Banach space $X$ and let the mapping $B_{i}: C \rightarrow X$ be $\lambda_{i}$-strictly pseudocontractive and $\alpha_{i}$-strongly accretive with $\alpha_{i}+\lambda_{i} \geq 1$ for $i=1$, 2 . Then, for $\mu_{i} \in(0,1]$, we have

$$
\left\|\left(I-\mu_{i} B_{i}\right) x-\left(I-\mu_{i} B_{i}\right) y\right\| \leq\left\{\sqrt{\frac{1-\alpha_{i}}{\lambda_{i}}}+\left(1-\mu_{i}\right)\left(1+\frac{1}{\lambda_{i}}\right)\right\}\|x-y\|, \quad \forall x, y \in C,
$$

for $i=1$, 2. In particular, if $1-\frac{\lambda_{i}}{1+\lambda_{i}}\left(1-\sqrt{\frac{1-\alpha_{i}}{\lambda_{i}}}\right) \leq \mu_{i} \leq 1$, then $I-\mu_{i} B_{i}$ is nonexpansive for $i=1,2$.

Proof Taking into account the $\lambda_{i}$-strictly pseudocontractivity of $B_{i}$, we derive for every $x, y \in C$

$$
\begin{aligned}
\lambda_{i}\left\|\left(I-B_{i}\right) x-\left(I-B_{i}\right) y\right\|^{2} & \leq\left\langle\left(I-B_{i}\right) x-\left(I-B_{i}\right) y, J(x-y)\right\rangle \\
& \leq\left\|\left(I-B_{i}\right) x-\left(I-B_{i}\right) y\right\|\|x-y\|,
\end{aligned}
$$

which implies that

$$
\left\|\left(I-B_{i}\right) x-\left(I-B_{i}\right) y\right\| \leq \frac{1}{\lambda_{i}}\|x-y\| .
$$


Hence,

$$
\left\|B_{i} x-B_{i} y\right\| \leq\left\|\left(I-B_{i}\right) x-\left(I-B_{i}\right) y\right\|+\|x-y\| \leq\left(1+\frac{1}{\lambda_{i}}\right)\|x-y\| .
$$

Utilizing the $\alpha_{i}$-strong accretivity and $\lambda_{i}$-strict pseudocontractivity of $B_{i}$, we get

$$
\lambda_{i}\left\|\left(I-B_{i}\right) x-\left(I-B_{i}\right) y\right\|^{2} \leq\|x-y\|^{2}-\left\langle B_{i} x-B_{i} y, J(x-y)\right\rangle \leq\left(1-\alpha_{i}\right)\|x-y\|^{2} .
$$

So, we have

$$
\left\|\left(I-B_{i}\right) x-\left(I-B_{i}\right) y\right\| \leq \sqrt{\frac{1-\alpha_{i}}{\lambda_{i}}}\|x-y\| .
$$

Therefore, for $\mu_{i} \in(0,1]$ we have

$$
\begin{aligned}
\left\|\left(I-\mu_{i} B_{i}\right) x-\left(I-\mu_{i} B_{i}\right) y\right\| & \leq\left\|\left(I-B_{i}\right) x-\left(I-B_{i}\right) y\right\|+\left(1-\mu_{i}\right)\left\|B_{i} x-B_{i} y\right\| \\
& \leq \sqrt{\frac{1-\alpha_{i}}{\lambda_{i}}}\|x-y\|+\left(1-\mu_{i}\right)\left(1+\frac{1}{\lambda_{i}}\right)\|x-y\| \\
& =\left\{\sqrt{\frac{1-\alpha_{i}}{\lambda_{i}}}+\left(1-\mu_{i}\right)\left(1+\frac{1}{\lambda_{i}}\right)\right\}\|x-y\| .
\end{aligned}
$$

Since $1-\frac{\lambda_{i}}{1+\lambda_{i}}\left(1-\sqrt{\frac{1-\alpha_{i}}{\lambda_{i}}}\right) \leq \mu_{i} \leq 1$, it follows immediately that

$$
\sqrt{\frac{1-\alpha_{i}}{\lambda_{i}}}+\left(1-\mu_{i}\right)\left(1+\frac{1}{\lambda_{i}}\right) \leq 1
$$

This implies that $I-\mu_{i} B_{i}$ is nonexpansive for $i=1,2$.

Lemma 2.10 Let $C$ be a nonempty closed convex subset of a smooth Banach space $X$. Let $\Pi_{C}$ be a sunny nonexpansive retraction from $X$ onto $C$, and let the mapping $B_{i}: C \rightarrow X$ be $\lambda_{i}$-strictly pseudocontractive and $\alpha_{i}$-strongly accretive with $\alpha_{i}+\lambda_{i} \geq 1$ for $i=1,2$. Let $G: C \rightarrow C$ be a mapping defined by

$$
G(x)=\Pi_{C}\left[\Pi_{C}\left(x-\mu_{2} B_{2} x\right)-\mu_{1} B_{1} \Pi_{C}\left(x-\mu_{2} B_{2} x\right)\right], \quad \forall x \in C .
$$

If $1-\frac{\lambda_{i}}{1+\lambda_{i}}\left(1-\sqrt{\frac{1-\alpha_{i}}{\lambda_{i}}}\right) \leq \mu_{i} \leq 1$, then $G: C \rightarrow C$ is nonexpansive.

Proof According to Lemma 2.9, we know that $I-\mu_{i} B_{i}$ is nonexpansive for $i=1,2$. Hence, for all $x, y \in C$, we have

$$
\begin{aligned}
\|G(x)-G(y)\|= & \| \Pi_{C}\left[\Pi_{C}\left(x-\mu_{2} B_{2} x\right)-\mu_{1} B_{1} \Pi_{C}\left(x-\mu_{2} B_{2} x\right)\right] \\
& -\Pi_{C}\left[\Pi_{C}\left(y-\mu_{2} B_{2} y\right)-\mu_{1} B_{1} \Pi_{C}\left(y-\mu_{2} B_{2} y\right)\right] \| \\
= & \left\|\Pi_{C}\left(I-\mu_{1} B_{1}\right) \Pi_{C}\left(I-\mu_{2} B_{2}\right) x-\Pi_{C}\left(I-\mu_{1} B_{1}\right) \Pi_{C}\left(I-\mu_{2} B_{2}\right) y\right\|
\end{aligned}
$$




$$
\begin{aligned}
& \leq\left\|\left(I-\mu_{1} B_{1}\right) \Pi_{C}\left(I-\mu_{2} B_{2}\right) x-\left(I-\mu_{1} B_{1}\right) \Pi_{C}\left(I-\mu_{2} B_{2}\right) y\right\| \\
& \leq\left\|\Pi_{C}\left(I-\mu_{2} B_{2}\right) x-\Pi_{C}\left(I-\mu_{2} B_{2}\right) y\right\| \\
& \leq\left\|\left(I-\mu_{2} B_{2}\right) x-\left(I-\mu_{2} B_{2}\right) y\right\| \\
& \leq\|x-y\| .
\end{aligned}
$$

This shows that $G: C \rightarrow C$ is nonexpansive. This completes the proof.

Lemma 2.11 Let $C$ be a nonempty closed convex subset of a smooth Banach space X. Let $\Pi_{C}$ be a sunny nonexpansive retraction from $X$ onto $C$, and let the mapping $B_{i}: C \rightarrow X$ be $\lambda_{i}$-strictly pseudocontractive and $\alpha_{i}$-strongly accretive for $i=1,2$. For given $x^{*}, y^{*} \in C$, $\left(x^{*}, y^{*}\right)$ is a solution of GSVI (1.4) if and only if $x^{*}=\Pi_{C}\left(y^{*}-\mu_{1} B_{1} y^{*}\right)$, where $y^{*}=\Pi_{C}\left(x^{*}-\right.$ $\left.\mu_{2} B_{2} x^{*}\right)$.

Proof We can rewrite GSVI (1.4) as

$$
\begin{cases}\left\langle x^{*}-\left(y^{*}-\mu_{1} B_{1} y^{*}\right), J\left(x-x^{*}\right)\right\rangle \geq 0, & \forall x \in C, \\ \left\langle y^{*}-\left(x^{*}-\mu_{2} B_{2} x^{*}\right), J\left(x-y^{*}\right)\right\rangle \geq 0, & \forall x \in C,\end{cases}
$$

which is obviously equivalent to

$$
\left\{\begin{array}{l}
x^{*}=\Pi_{C}\left(y^{*}-\mu_{1} B_{1} y^{*}\right), \\
y^{*}=\Pi_{C}\left(x^{*}-\mu_{2} B_{2} x^{*}\right),
\end{array}\right.
$$

because of Lemma 2.4. This completes the proof.

Remark 2.1 By Lemma 2.11, we observe that

$$
x^{*}=\Pi_{C}\left[\Pi_{C}\left(x^{*}-\mu_{2} B_{2} x^{*}\right)-\mu_{1} B_{1} \Pi_{C}\left(x^{*}-\mu_{2} B_{2} x^{*}\right)\right],
$$

which implies that $x^{*}$ is a fixed point of the mapping $G=\Pi_{C}\left(I-\mu_{1} B_{1}\right) \Pi_{C}\left(I-\mu_{2} B_{2}\right)$.

\section{Two-step implicit iterative algorithm}

In this section, we introduce our two-step implicit iterative algorithm and show the strong convergence of the purposed algorithm.

Theorem 3.1 Let $C$ be a nonempty closed convex subset of a uniformly convex Banach space $X$ which has a uniformly Gâteaux differentiable norm. Let $\Pi_{C}$ be a sunny nonexpansive retraction from $X$ onto $C$. Let the mapping $B_{i}: C \rightarrow X$ be $\lambda_{i}$-strictly pseudocontractive and $\alpha_{i}$-strongly accretive with $\alpha_{i}+\lambda_{i} \geq 1$ for $i=1,2$. Let $f: C \rightarrow C$ be a contraction with coefficient $\rho \in(0,1)$. Let $\left\{S_{n}\right\}_{n=1}^{\infty}$ be an infinite family of nonexpansive mappings of $C$ into itself such that $F=\bigcap_{i=1}^{\infty} \operatorname{Fix}\left(S_{i}\right) \cap \Omega \neq \emptyset$, where $\Omega$ is the fixed point set of the mapping $G=\Pi_{C}\left(I-\mu_{1} B_{1}\right) \Pi_{C}\left(I-\mu_{2} B_{2}\right)$. For arbitrarily given $x_{0} \in C$, let $\left\{x_{n}\right\}$ be the sequence generated by

$$
\left\{\begin{array}{l}
y_{n}=\alpha_{n} f\left(x_{n}\right)+\left(1-\alpha_{n}\right) G\left(x_{n}\right), \\
x_{n+1}=\beta_{n} x_{n}+\gamma_{n} y_{n}+\delta_{n} S_{n+1} x_{n+1}, \quad \forall n \geq 0,
\end{array}\right.
$$


where $1-\frac{\lambda_{i}}{1+\lambda_{i}}\left(1-\sqrt{\frac{1-\alpha_{i}}{\lambda_{i}}}\right) \leq \mu_{i} \leq 1$ for $i=1,2$. Suppose that $\left\{\alpha_{n}\right\},\left\{\beta_{n}\right\},\left\{\gamma_{n}\right\}$ and $\left\{\delta_{n}\right\}$ are the sequences in $(0,1)$ satisfying the following conditions:

(i) $\lim _{n \rightarrow \infty} \alpha_{n}=0$ and $\sum_{n=0}^{\infty} \alpha_{n}=\infty$;

(ii) $\beta_{n}+\gamma_{n}+\delta_{n}=1, \forall n \geq 0$, and $\liminf _{n \rightarrow \infty} \delta_{n}>0$;

(iii) $0<\liminf _{n \rightarrow \infty} \beta_{n} \leq \limsup _{n \rightarrow \infty}\left(\beta_{n}+\delta_{n}\right)<1$;

(iv) $\sum_{n=1}^{\infty}\left|\frac{\gamma_{n}}{1-\beta_{n}}-\frac{\gamma_{n-1}}{1-\beta_{n-1}}\right|<\infty$ or $\lim _{n \rightarrow \infty} \frac{1}{\alpha_{n}}\left|\frac{\gamma_{n}}{1-\beta_{n}}-\frac{\gamma_{n-1}}{1-\beta_{n-1}}\right|=0$;

(v) $\sum_{n=1}^{\infty}\left|\alpha_{n}-\alpha_{n-1}\right|<\infty$ or $\lim _{n \rightarrow \infty} \alpha_{n-1} / \alpha_{n}=1$;

(vi) $\sum_{n=1}^{\infty}\left|\beta_{n}-\beta_{n-1}\right|<\infty$ or $\lim _{n \rightarrow \infty}\left|\beta_{n}-\beta_{n-1}\right| / \alpha_{n}=0$.

Assume that $\sum_{n=1}^{\infty} \sup _{x \in D}\left\|S_{n} x-S_{n-1} x\right\|<\infty$ for any bounded subset $D$ of $C$, and let $S$ be a mapping of $C$ into itself defined by $S x=\lim _{n \rightarrow \infty} S_{n} x$ for all $x \in C$, and suppose that $\operatorname{Fix}(S)=$ $\bigcap_{i=1}^{\infty} \operatorname{Fix}\left(S_{i}\right)$. Then $\left\{x_{n}\right\}$ converges strongly to $q \in F$, which solves the following VIP:

$$
\langle q-f(q), J(q-p)\rangle \leq 0, \quad \forall p \in F
$$

Proof Take a fixed $p \in F$ arbitrarily. Then by Lemma 2.11 we know that $p=G(p)$. Moreover, by Lemma 2.10 we have

$$
\begin{aligned}
\left\|y_{n}-p\right\| & =\left\|\alpha_{n}\left(f\left(x_{n}\right)-p\right)+\left(1-\alpha_{n}\right)\left(G\left(x_{n}\right)-p\right)\right\| \\
& \leq \alpha_{n}\left\|f\left(x_{n}\right)-f(p)\right\|+\alpha_{n}\|f(p)-p\|+\left(1-\alpha_{n}\right)\left\|G\left(x_{n}\right)-p\right\| \\
& \leq \alpha_{n} \rho\left\|x_{n}-p\right\|+\alpha_{n}\|f(p)-p\|+\left(1-\alpha_{n}\right)\left\|x_{n}-p\right\| \\
& =\left(1-\alpha_{n}(1-\rho)\right)\left\|x_{n}-p\right\|+\alpha_{n}\|f(p)-p\| .
\end{aligned}
$$

From (3.3) we obtain

$$
\begin{aligned}
\left\|x_{n+1}-p\right\| & =\left\|\beta_{n}\left(x_{n}-p\right)+\gamma_{n}\left(y_{n}-p\right)+\delta_{n}\left(S_{n+1} x_{n+1}-p\right)\right\| \\
& \leq \beta_{n}\left\|x_{n}-p\right\|+\gamma_{n}\left\|y_{n}-p\right\|+\delta_{n}\left\|x_{n+1}-p\right\|,
\end{aligned}
$$

which together with (3.3) implies that

$$
\begin{aligned}
\left\|x_{n+1}-p\right\| & \leq \frac{1}{\beta_{n}+\gamma_{n}}\left[\beta_{n}\left\|x_{n}-p\right\|+\gamma_{n}\left\|y_{n}-p\right\|\right] \\
& \leq \frac{1}{\beta_{n}+\gamma_{n}}\left\{\beta_{n}\left\|x_{n}-p\right\|+\gamma_{n}\left[\left(1-\alpha_{n}(1-\rho)\right)\left\|x_{n}-p\right\|+\alpha_{n}\|f(p)-p\|\right]\right\} \\
& =\frac{1}{\beta_{n}+\gamma_{n}}\left\{\left(\beta_{n}+\gamma_{n}-\alpha_{n} \gamma_{n}(1-\rho)\right)\left\|x_{n}-p\right\|+\alpha_{n} \gamma_{n}\|f(p)-p\|\right\} \\
& =\left(1-\frac{\alpha_{n} \gamma_{n}(1-\rho)}{\beta_{n}+\gamma_{n}}\right)\left\|x_{n}-p\right\|+\frac{\alpha_{n} \gamma_{n}}{\beta_{n}+\gamma_{n}}\|f(p)-p\| \\
& =\left(1-\frac{\alpha_{n} \gamma_{n}(1-\rho)}{\beta_{n}+\gamma_{n}}\right)\left\|x_{n}-p\right\|+\frac{\alpha_{n} \gamma_{n}(1-\rho)}{\beta_{n}+\gamma_{n}} \cdot \frac{\|f(p)-p\|}{1-\rho} \\
& \leq \max \left\{\left\|x_{0}-p\right\|, \frac{\|f(p)-p\|}{1-\rho}\right\},
\end{aligned}
$$

which implies that $\left\{x_{n}\right\}$ is bounded. By Lemma 2.10 we know from (3.3) that $\left\{G\left(x_{n}\right)\right\}$ and $\left\{y_{n}\right\}$ both are bounded. 
Let us show that $\left\|x_{n+1}-x_{n}\right\| \rightarrow 0$ and $\left\|x_{n}-y_{n}\right\| \rightarrow 0$ as $n \rightarrow \infty$. As a matter of fact, from (3.1) we have

$$
y_{n}-y_{n-1}=\alpha_{n}\left(f\left(x_{n}\right)-f\left(x_{n-1}\right)\right)+\left(\alpha_{n}-\alpha_{n-1}\right)\left(f\left(x_{n-1}\right)-G\left(x_{n-1}\right)\right)+\left(1-\alpha_{n}\right)\left(G\left(x_{n}\right)-G\left(x_{n-1}\right)\right) .
$$

It follows that

$$
\begin{aligned}
\left\|y_{n}-y_{n-1}\right\| \leq & \alpha_{n}\left\|f\left(x_{n}\right)-f\left(x_{n-1}\right)\right\|+\left|\alpha_{n}-\alpha_{n-1}\right|\left\|f\left(x_{n-1}\right)-G\left(x_{n-1}\right)\right\| \\
& +\left(1-\alpha_{n}\right)\left\|G\left(x_{n}\right)-G\left(x_{n-1}\right)\right\| \\
& \leq \alpha_{n} \rho\left\|x_{n}-x_{n-1}\right\|+\left|\alpha_{n}-\alpha_{n-1}\right|\left\|f\left(x_{n-1}\right)-G\left(x_{n-1}\right)\right\| \\
& +\left(1-\alpha_{n}\right)\left\|x_{n}-x_{n-1}\right\| \\
= & \left(1-(1-\rho) \alpha_{n}\right)\left\|x_{n}-x_{n-1}\right\|+\left|\alpha_{n}-\alpha_{n-1}\right|\left\|f\left(x_{n-1}\right)-G\left(x_{n-1}\right)\right\| .
\end{aligned}
$$

Taking into consideration that $0<\liminf _{n \rightarrow \infty} \beta_{n} \leq \limsup _{n \rightarrow \infty}\left(\beta_{n}+\delta_{n}\right)<1$ and $\liminf _{n \rightarrow \infty} \gamma_{n}=1-\limsup _{n \rightarrow \infty}\left(\beta_{n}+\delta_{n}\right)>0$, we may assume that $\left\{\beta_{n}\right\} \subset[c, d]$ and $\gamma_{n} \subset$ $[c, 1)$ for some $c, d \in(0,1)$. First, we write $x_{n}=\beta_{n-1} x_{n-1}+\left(1-\beta_{n-1}\right) v_{n-1}, \forall n \geq 1$, where $v_{n-1}=\frac{x_{n}-\beta_{n-1} x_{n-1}}{1-\beta_{n-1}}$. It follows that for all $n \geq 1$,

$$
\begin{aligned}
v_{n}-v_{n-1}= & \frac{x_{n+1}-\beta_{n} x_{n}}{1-\beta_{n}}-\frac{x_{n}-\beta_{n-1} x_{n-1}}{1-\beta_{n-1}} \\
= & \frac{\gamma_{n} y_{n}+\delta_{n} S_{n+1} x_{n+1}}{1-\beta_{n}}-\frac{\gamma_{n-1} y_{n-1}+\delta_{n-1} S_{n} x_{n}}{1-\beta_{n-1}} \\
= & \frac{\gamma_{n}\left(y_{n}-y_{n-1}\right)+\delta_{n}\left(S_{n+1} x_{n+1}-S_{n} x_{n}\right)}{1-\beta_{n}} \\
& +\left(\frac{\gamma_{n}}{1-\beta_{n}}-\frac{\gamma_{n-1}}{1-\beta_{n-1}}\right) y_{n-1}+\left(\frac{\delta_{n}}{1-\beta_{n}}-\frac{\delta_{n-1}}{1-\beta_{n-1}}\right) S_{n} x_{n} .
\end{aligned}
$$

This together with (3.4) implies that

$$
\begin{aligned}
\| v_{n}- & v_{n-1} \| \\
\leq & \frac{\left\|\gamma_{n}\left(y_{n}-y_{n-1}\right)+\delta_{n}\left(S_{n+1} x_{n+1}-S_{n} x_{n}\right)\right\|}{1-\beta_{n}}+\left|\frac{\gamma_{n}}{1-\beta_{n}}-\frac{\gamma_{n-1}}{1-\beta_{n-1}}\right|\left\|y_{n-1}\right\| \\
& +\left|\frac{\delta_{n}}{1-\beta_{n}}-\frac{\delta_{n-1}}{1-\beta_{n-1}}\right|\left\|S_{n} x_{n}\right\| \\
\leq & \frac{\gamma_{n}\left\|y_{n}-y_{n-1}\right\|+\delta_{n}\left(\left\|S_{n+1} x_{n+1}-S_{n+1} x_{n}\right\|+\left\|S_{n+1} x_{n}-S_{n} x_{n}\right\|\right)}{1-\beta_{n}} \\
& +\left|\frac{\gamma_{n}}{1-\beta_{n}}-\frac{\gamma_{n-1}}{1-\beta_{n-1}}\right|\left\|y_{n-1}\right\|+\left|\frac{\gamma_{n}}{1-\beta_{n}}-\frac{\gamma_{n-1}}{1-\beta_{n-1}}\right|\left\|S_{n} x_{n}\right\| \\
\leq & \frac{\gamma_{n}\left\|y_{n}-y_{n-1}\right\|+\delta_{n}\left\|x_{n+1}-x_{n}\right\|}{1-\beta_{n}}+\frac{\delta_{n}}{1-\beta_{n}}\left\|S_{n+1} x_{n}-S_{n} x_{n}\right\| \\
& +\left|\frac{\gamma_{n}}{1-\beta_{n}}-\frac{\gamma_{n-1}}{1-\beta_{n-1}}\right|\left(\left\|y_{n-1}\right\|+\left\|S_{n} x_{n}\right\|\right) \\
\leq & \frac{\gamma_{n}}{\gamma_{n}+\delta_{n}}\left[\left(1-(1-\rho) \alpha_{n}\right)\left\|x_{n}-x_{n-1}\right\|+\left|\alpha_{n}-\alpha_{n-1}\right|\left\|f\left(x_{n-1}\right)-G\left(x_{n-1}\right)\right\|\right]
\end{aligned}
$$




$$
\begin{aligned}
& +\frac{\delta_{n}}{\gamma_{n}+\delta_{n}}\left\|x_{n+1}-x_{n}\right\|+\frac{\delta_{n}}{1-\beta_{n}}\left\|S_{n+1} x_{n}-S_{n} x_{n}\right\| \\
& +\left|\frac{\gamma_{n}}{1-\beta_{n}}-\frac{\gamma_{n-1}}{1-\beta_{n-1}}\right|\left(\left\|y_{n-1}\right\|+\left\|S_{n} x_{n}\right\|\right) \\
& \leq \frac{\gamma_{n}}{\gamma_{n}+\delta_{n}}\left(1-(1-\rho) \alpha_{n}\right)\left\|x_{n}-x_{n-1}\right\|+\frac{\gamma_{n}}{\gamma_{n}+\delta_{n}}\left|\alpha_{n}-\alpha_{n-1}\right| M \\
& +\frac{\delta_{n}}{\gamma_{n}+\delta_{n}}\left\|x_{n+1}-x_{n}\right\|+\frac{\delta_{n}}{\gamma_{n}+\delta_{n}}\left\|S_{n+1} x_{n}-S_{n} x_{n}\right\|+\left|\frac{\gamma_{n}}{1-\beta_{n}}-\frac{\gamma_{n-1}}{1-\beta_{n-1}}\right| M,
\end{aligned}
$$

where $\sup _{n \geq 0}\left\{\left\|f\left(x_{n}\right)-G\left(x_{n}\right)\right\|\right\} \leq M$ and $\sup _{n \geq 1}\left\{\left\|y_{n-1}\right\|+\left\|S_{n} x_{n}\right\|\right\} \leq M$ for some $M>0$.

Now we observe that

$$
\begin{aligned}
& x_{n+1}-x_{n}=\left(\beta_{n}-\beta_{n-1}\right)\left(x_{n}-v_{n}\right)+\beta_{n-1}\left(x_{n}-x_{n-1}\right)+\left(1-\beta_{n-1}\right)\left(v_{n}-v_{n-1}\right), \\
& 1-\left(1-\beta_{n-1}\right) \frac{\delta_{n}}{\gamma_{n}+\delta_{n}}=\frac{\gamma_{n}+\beta_{n-1} \delta_{n}}{\gamma_{n}+\delta_{n}},
\end{aligned}
$$

and

$$
\begin{aligned}
\beta_{n-1} & +\left(1-\beta_{n-1}\right) \frac{\gamma_{n}}{\gamma_{n}+\delta_{n}}\left(1-(1-\rho) \alpha_{n}\right) \\
= & \beta_{n-1}\left(\frac{\gamma_{n}}{\gamma_{n}+\delta_{n}}+\frac{\delta_{n}}{\gamma_{n}+\delta_{n}}\right)+\left(1-\beta_{n-1}\right) \frac{\gamma_{n}}{\gamma_{n}+\delta_{n}}\left(1-(1-\rho) \alpha_{n}\right) \\
= & \frac{\beta_{n-1} \delta_{n}}{\gamma_{n}+\delta_{n}}+\frac{\gamma_{n}}{\gamma_{n}+\delta_{n}}\left[\beta_{n-1}+\left(1-\beta_{n-1}\right)\left(1-(1-\rho) \alpha_{n}\right)\right] \\
= & \frac{\beta_{n-1} \delta_{n}}{\gamma_{n}+\delta_{n}}+\frac{\gamma_{n}}{\gamma_{n}+\delta_{n}}\left[1-\left(1-\beta_{n-1}\right)(1-\rho) \alpha_{n}\right] \\
= & \frac{\gamma_{n}+\beta_{n-1} \delta_{n}}{\gamma_{n}+\delta_{n}}-\left(1-\beta_{n-1}\right)(1-\rho) \frac{\alpha_{n} \gamma_{n}}{\gamma_{n}+\delta_{n}} .
\end{aligned}
$$

Hence from (3.6) it follows that

$$
\begin{aligned}
& \left\|x_{n+1}-x_{n}\right\| \\
& \leq\left|\beta_{n}-\beta_{n-1}\right|\left\|x_{n}-v_{n}\right\|+\beta_{n-1}\left\|x_{n}-x_{n-1}\right\|+\left(1-\beta_{n-1}\right)\left\|v_{n}-v_{n-1}\right\| \\
& \leq\left|\beta_{n}-\beta_{n-1}\right|\left\|x_{n}-v_{n}\right\|+\beta_{n-1}\left\|x_{n}-x_{n-1}\right\| \\
& +\left(1-\beta_{n-1}\right)\left\{\frac{\gamma_{n}}{\gamma_{n}+\delta_{n}}\left(1-(1-\rho) \alpha_{n}\right)\left\|x_{n}-x_{n-1}\right\|+\frac{\gamma_{n}}{\gamma_{n}+\delta_{n}}\left|\alpha_{n}-\alpha_{n-1}\right| M\right. \\
& \left.+\frac{\delta_{n}}{\gamma_{n}+\delta_{n}}\left\|x_{n+1}-x_{n}\right\|+\frac{\delta_{n}}{\gamma_{n}+\delta_{n}}\left\|S_{n+1} x_{n}-S_{n} x_{n}\right\|+\left|\frac{\gamma_{n}}{1-\beta_{n}}-\frac{\gamma_{n-1}}{1-\beta_{n-1}}\right| M\right\} \\
& \leq\left|\beta_{n}-\beta_{n-1}\right| M_{1}+\left[\beta_{n-1}+\left(1-\beta_{n-1}\right) \frac{\gamma_{n}}{\gamma_{n}+\delta_{n}}\left(1-(1-\rho) \alpha_{n}\right)\right]\left\|x_{n}-x_{n-1}\right\| \\
& +\left|\alpha_{n}-\alpha_{n-1}\right| M_{1}+\left(1-\beta_{n-1}\right) \frac{\delta_{n}}{\gamma_{n}+\delta_{n}}\left\|x_{n+1}-x_{n}\right\|+\left\|S_{n+1} x_{n}-S_{n} x_{n}\right\| \\
& +\left|\frac{\gamma_{n}}{1-\beta_{n}}-\frac{\gamma_{n-1}}{1-\beta_{n-1}}\right| M_{1} \\
& =\left[\frac{\gamma_{n}+\beta_{n-1} \delta_{n}}{\gamma_{n}+\delta_{n}}-\left(1-\beta_{n-1}\right)(1-\rho) \frac{\alpha_{n} \gamma_{n}}{\gamma_{n}+\delta_{n}}\right]\left\|x_{n}-x_{n-1}\right\|
\end{aligned}
$$




$$
\begin{aligned}
& +\left(1-\beta_{n-1}\right) \frac{\delta_{n}}{\gamma_{n}+\delta_{n}}\left\|x_{n+1}-x_{n}\right\| \\
& +M_{1}\left(\left|\alpha_{n}-\alpha_{n-1}\right|+\left|\beta_{n}-\beta_{n-1}\right|+\left|\frac{\gamma_{n}}{1-\beta_{n}}-\frac{\gamma_{n-1}}{1-\beta_{n-1}}\right|\right)+\left\|S_{n+1} x_{n}-S_{n} x_{n}\right\|,
\end{aligned}
$$

where $\sup _{n \geq 0}\left\{M+\left\|x_{n}-v_{n}\right\|\right\} \leq M_{1}$ for some $M_{1}>0$. Therefore, we get

$$
\begin{aligned}
& \left\|x_{n+1}-x_{n}\right\| \\
& \leq\left[1-\left(1-\beta_{n-1}\right)(1-\rho) \frac{\alpha_{n} \gamma_{n}}{\gamma_{n}+\beta_{n-1} \delta_{n}}\right]\left\|x_{n}-x_{n-1}\right\| \\
& +\frac{\gamma_{n}+\delta_{n}}{\gamma_{n}+\beta_{n-1} \delta_{n}}\left[M_{1}\left(\left|\alpha_{n}-\alpha_{n-1}\right|+\left|\beta_{n}-\beta_{n-1}\right|+\left|\frac{\gamma_{n}}{1-\beta_{n}}-\frac{\gamma_{n-1}}{1-\beta_{n-1}}\right|\right)\right. \\
& \left.+\left\|S_{n+1} x_{n}-S_{n} x_{n}\right\|\right] \\
& \leq\left[1-c(1-d)(1-\rho) \frac{\alpha_{n}}{\gamma_{n}+\beta_{n-1} \delta_{n}}\right]\left\|x_{n}-x_{n-1}\right\| \\
& +\frac{1}{\gamma_{n}+\beta_{n-1} \delta_{n}}\left[M_{1}\left(\left|\alpha_{n}-\alpha_{n-1}\right|+\left|\beta_{n}-\beta_{n-1}\right|+\left|\frac{\gamma_{n}}{1-\beta_{n}}-\frac{\gamma_{n-1}}{1-\beta_{n-1}}\right|\right)\right. \\
& \left.+\left\|S_{n+1} x_{n}-S_{n} x_{n}\right\|\right] \\
& =\left[1-c(1-d)(1-\rho) \frac{\alpha_{n}}{\gamma_{n}+\beta_{n-1} \delta_{n}}\right]\left\|x_{n}-x_{n-1}\right\| \\
& +c(1-d)(1-\rho) \frac{1}{\gamma_{n}+\beta_{n-1} \delta_{n}} \cdot \frac{1}{c(1-d)(1-\rho)}\left[M _ { 1 } \left(\left|\alpha_{n}-\alpha_{n-1}\right|+\left|\beta_{n}-\beta_{n-1}\right|\right.\right. \\
& \left.\left.+\left|\frac{\gamma_{n}}{1-\beta_{n}}-\frac{\gamma_{n-1}}{1-\beta_{n-1}}\right|\right)+\left\|S_{n+1} x_{n}-S_{n} x_{n}\right\|\right] \text {. }
\end{aligned}
$$

Utilizing Lemma 2.1, from conditions (i), (iv)-(vi) and the assumption on $\left\{S_{n}\right\}$, we deduce that

$$
\lim _{n \rightarrow \infty}\left\|x_{n+1}-x_{n}\right\|=0
$$

Also, we note that for $p \in F$,

$$
\begin{aligned}
\left\|y_{n}-p\right\|^{2} & =\left\|\alpha_{n}\left(f\left(x_{n}\right)-p\right)+\left(1-\alpha_{n}\right)\left(G\left(x_{n}\right)-G(p)\right)\right\|^{2} \\
& \leq\left\|\left(1-\alpha_{n}\right)\left(G\left(x_{n}\right)-G(p)\right)\right\|^{2}+2 \alpha_{n}\left\langle f\left(x_{n}\right)-p, J\left(y_{n}-p\right)\right\rangle \\
& \leq\left\|x_{n}-p\right\|^{2}+2 \alpha_{n}\left\|f\left(x_{n}\right)-p\right\|\left\|y_{n}-p\right\| .
\end{aligned}
$$

Since $\left\{x_{n}\right\}$ and $\left\{y_{n}\right\}$ both are bounded, by Lemma 2.5 there exists a continuous strictly increasing function $g:[0, \infty) \rightarrow[0, \infty), g(0)=0$ such that

$$
\begin{aligned}
& \left\|x_{n+1}-p\right\|^{2} \\
& \quad=\left\|\left(\beta_{n}+\delta_{n}\right)\left(\frac{\beta_{n}}{\beta_{n}+\delta_{n}}\left(x_{n}-p\right)+\frac{\delta_{n}}{\beta_{n}+\delta_{n}}\left(S_{n+1} x_{n+1}-p\right)\right)+\gamma_{n}\left(y_{n}-p\right)\right\|^{2}
\end{aligned}
$$




$$
\begin{aligned}
\leq & \left(\beta_{n}+\delta_{n}\right)\left\|\frac{\beta_{n}}{\beta_{n}+\delta_{n}}\left(x_{n}-p\right)+\frac{\delta_{n}}{\beta_{n}+\delta_{n}}\left(S_{n+1} x_{n+1}-p\right)\right\|^{2}+\gamma_{n}\left\|y_{n}-p\right\|^{2} \\
\leq & \left(\beta_{n}+\delta_{n}\right)\left[\frac{\beta_{n}}{\beta_{n}+\delta_{n}}\left\|x_{n}-p\right\|^{2}+\frac{\delta_{n}}{\beta_{n}+\delta_{n}}\left\|S_{n+1} x_{n+1}-p\right\|\right. \\
& \left.-\frac{\beta_{n} \delta_{n}}{\left(\beta_{n}+\delta_{n}\right)^{2}} g\left(\left\|x_{n}-S_{n+1} x_{n+1}\right\|\right)\right]+\gamma_{n}\left\|y_{n}-p\right\|^{2} \\
\leq & \beta_{n}\left\|x_{n}-p\right\|^{2}+\delta_{n}\left\|x_{n+1}-p\right\|^{2}-\frac{\beta_{n} \delta_{n}}{\beta_{n}+\delta_{n}} g\left(\left\|x_{n}-S_{n+1} x_{n+1}\right\|\right)+\gamma_{n}\left\|y_{n}-p\right\|^{2} \\
\leq & \beta_{n}\left\|x_{n}-p\right\|^{2}+\delta_{n}\left\|x_{n+1}-p\right\|^{2}-\frac{\beta_{n} \delta_{n}}{\beta_{n}+\delta_{n}} g\left(\left\|x_{n}-S_{n+1} x_{n+1}\right\|\right) \\
& +\gamma_{n}\left[\left\|x_{n}-p\right\|^{2}+2 \alpha_{n}\left\|f\left(x_{n}\right)-p\right\|\left\|y_{n}-p\right\|\right] \\
= & \left(\beta_{n}+\gamma_{n}\right)\left\|x_{n}-p\right\|^{2}+\delta_{n}\left\|x_{n+1}-p\right\|^{2}-\frac{\beta_{n} \delta_{n}}{\beta_{n}+\delta_{n}} g\left(\left\|x_{n}-S_{n+1} x_{n+1}\right\|\right) \\
& +2 \alpha_{n} \gamma_{n}\left\|f\left(x_{n}\right)-p\right\|\left\|y_{n}-p\right\|,
\end{aligned}
$$

which immediately yields

$$
\begin{aligned}
& \beta_{n} \delta_{n} g\left(\left\|x_{n}-S_{n+1} x_{n+1}\right\|\right) \\
& \quad \leq \frac{\beta_{n} \delta_{n}}{\beta_{n}+\delta_{n}} g\left(\left\|x_{n}-S_{n+1} x_{n+1}\right\|\right) \\
& \quad \leq\left(\beta_{n}+\gamma_{n}\right)\left(\left\|x_{n}-p\right\|^{2}-\left\|x_{n+1}-p\right\|^{2}\right)+2 \alpha_{n}\left\|f\left(x_{n}\right)-p\right\|\left\|y_{n}-p\right\| \\
& \quad \leq\left(\left\|x_{n}-p\right\|+\left\|x_{n+1}-p\right\|\right)\left\|x_{n}-x_{n+1}\right\|+2 \alpha_{n}\left\|f\left(x_{n}\right)-p\right\|\left\|y_{n}-p\right\| .
\end{aligned}
$$

Since $\alpha_{n} \rightarrow 0,\left\|x_{n+1}-x_{n}\right\| \rightarrow 0, \liminf _{n \rightarrow \infty} \beta_{n}>0$ and $\liminf _{n \rightarrow \infty} \delta_{n}>0$, we get $\lim _{n \rightarrow \infty} g\left(\left\|x_{n}-S_{n+1} x_{n+1}\right\|\right)=0$ and hence

$$
\lim _{n \rightarrow \infty}\left\|x_{n}-S_{n+1} x_{n+1}\right\|=0 .
$$

In the meantime, according to condition (iii), we have

$$
\liminf _{n \rightarrow \infty} \gamma_{n}=\liminf _{n \rightarrow \infty}\left(1-\beta_{n}-\delta_{n}\right)=1-\limsup _{n \rightarrow \infty}\left(\beta_{n}+\delta_{n}\right)>0 .
$$

Thus, from (3.7) and (3.9) it follows that

$$
\begin{aligned}
\gamma_{n}\left\|y_{n}-x_{n}\right\| & =\left\|\left(x_{n+1}-x_{n}\right)-\delta_{n}\left(S_{n+1} x_{n+1}-x_{n}\right)\right\| \\
& \leq\left\|x_{n+1}-x_{n}\right\|+\delta_{n}\left\|S_{n+1} x_{n+1}-x_{n}\right\| \rightarrow 0 \quad \text { as } n \rightarrow \infty .
\end{aligned}
$$

That is,

$$
\lim _{n \rightarrow \infty}\left\|y_{n}-x_{n}\right\|=0 .
$$

This together with (3.1) leads to

$$
\begin{aligned}
\left(1-\alpha_{n}\right)\left\|G\left(x_{n}\right)-x_{n}\right\| & =\left\|\left(y_{n}-x_{n}\right)-\alpha_{n}\left(f\left(x_{n}\right)-x_{n}\right)\right\| \\
& \leq\left\|y_{n}-x_{n}\right\|+\alpha_{n}\left\|f\left(x_{n}\right)-x_{n}\right\| \rightarrow 0 \quad \text { as } n \rightarrow \infty .
\end{aligned}
$$


That is,

$$
\lim _{n \rightarrow \infty}\left\|x_{n}-G\left(x_{n}\right)\right\|=0 \text {. }
$$

On the other hand, we observe that

$$
y_{n}-G\left(x_{n}\right)=\alpha_{n}\left(f\left(x_{n}\right)-G\left(x_{n}\right)\right),
$$

which together with $\alpha_{n} \rightarrow 0$ implies that

$$
\lim _{n \rightarrow \infty}\left\|y_{n}-G\left(x_{n}\right)\right\|=0 \text {. }
$$

We note that

$$
\begin{aligned}
&\left\|S_{n} G\left(x_{n}\right)-G\left(x_{n}\right)\right\| \\
& \leq\left\|S_{n} G\left(x_{n}\right)-S_{n+1} x_{n+1}\right\|+\left\|S_{n+1} x_{n+1}-x_{n}\right\|+\left\|x_{n}-G\left(x_{n}\right)\right\| \\
& \leq\left\|S_{n} G\left(x_{n}\right)-S_{n} x_{n+1}\right\|+\left\|S_{n} x_{n+1}-S_{n+1} x_{n+1}\right\|+\left\|S_{n+1} x_{n+1}-x_{n}\right\|+\left\|x_{n}-G\left(x_{n}\right)\right\| \\
& \leq\left\|G\left(x_{n}\right)-x_{n+1}\right\|+\left\|S_{n} x_{n+1}-S_{n+1} x_{n+1}\right\|+\left\|S_{n+1} x_{n+1}-x_{n}\right\|+\left\|x_{n}-G\left(x_{n}\right)\right\| \\
& \leq\left\|G\left(x_{n}\right)-x_{n}\right\|+\left\|x_{n}-x_{n+1}\right\|+\left\|S_{n} x_{n+1}-S_{n+1} x_{n+1}\right\|+\left\|S_{n+1} x_{n+1}-x_{n}\right\| \\
&+\left\|x_{n}-G\left(x_{n}\right)\right\| \\
& \leq 2\left\|G\left(x_{n}\right)-x_{n}\right\|+\left\|x_{n}-x_{n+1}\right\|+\left\|S_{n} x_{n+1}-S_{n+1} x_{n+1}\right\|+\left\|S_{n+1} x_{n+1}-x_{n}\right\| .
\end{aligned}
$$

From (3.7), (3.9), (3.11) and the assumption on $\left\{S_{n}\right\}$, we obtain that

$$
\lim _{n \rightarrow \infty}\left\|S_{n} G\left(x_{n}\right)-G\left(x_{n}\right)\right\|=0
$$

By (3.13) and Lemma 2.6, we have

$$
\begin{aligned}
\left\|S G\left(x_{n}\right)-G\left(x_{n}\right)\right\| & \leq\left\|S G\left(x_{n}\right)-S_{n} G\left(x_{n}\right)\right\|+\left\|S_{n} G\left(x_{n}\right)-G\left(x_{n}\right)\right\| \\
& \rightarrow 0 \quad \text { as } n \rightarrow \infty .
\end{aligned}
$$

In terms of (3.11) and (3.14), we have

$$
\begin{aligned}
\left\|x_{n}-S x_{n}\right\| & \leq\left\|x_{n}-G\left(x_{n}\right)\right\|+\left\|G\left(x_{n}\right)-S G\left(x_{n}\right)\right\|+\left\|S G\left(x_{n}\right)-S x_{n}\right\| \\
& \leq 2\left\|x_{n}-G\left(x_{n}\right)\right\|+\left\|G\left(x_{n}\right)-S G\left(x_{n}\right)\right\| \\
& \rightarrow 0 \quad \text { as } n \rightarrow \infty .
\end{aligned}
$$

Define a mapping $W x=(1-\theta) S x+\theta G(x)$, where $G$ is defined by $(1.7), \theta \in(0,1)$ is a constant. Then by Lemma 2.8 we have that $\operatorname{Fix}(W)=\operatorname{Fix}(S) \cap \operatorname{Fix}(G)=F$. We observe that

$$
\begin{aligned}
\left\|x_{n}-W x_{n}\right\| & =\left\|(1-\theta)\left(x_{n}-S x_{n}\right)+\theta\left(x_{n}-G\left(x_{n}\right)\right)\right\| \\
& \leq(1-\theta)\left\|x_{n}-S x_{n}\right\|+\theta\left\|x_{n}-G\left(x_{n}\right)\right\| .
\end{aligned}
$$


From (3.11) and (3.15), we obtain

$$
\lim _{n \rightarrow \infty}\left\|x_{n}-W x_{n}\right\|=0
$$

Now, we claim that

$$
\limsup _{n \rightarrow \infty}\left\langle f(q)-q, J\left(x_{n}-q\right)\right\rangle \leq 0
$$

where $q=s-\lim _{t \rightarrow 0} x_{t}$ with $x_{t}$ being the fixed point of the contraction

$$
x \mapsto t f(x)+(1-t) W x
$$

Then $x_{t}$ solves the fixed point equation $x_{t}=t f\left(x_{t}\right)+(1-t) W x_{t}$. Thus we have

$$
\left\|x_{t}-x_{n}\right\|=\left\|(1-t)\left(W x_{t}-x_{n}\right)+t\left(f\left(x_{t}\right)-x_{n}\right)\right\| .
$$

By Lemma 2.2 we conclude that

$$
\begin{aligned}
\| x_{t}- & x_{n} \|^{2} \\
= & \left\|(1-t)\left(W x_{t}-x_{n}\right)+t\left(f\left(x_{t}\right)-x_{n}\right)\right\|^{2} \\
\leq & (1-t)^{2}\left\|W x_{t}-x_{n}\right\|^{2}+2 t\left\langle f\left(x_{t}\right)-x_{n}, J\left(x_{t}-x_{n}\right)\right\rangle \\
\leq & (1-t)^{2}\left(\left\|W x_{t}-W x_{n}\right\|+\left\|W x_{n}-x_{n}\right\|\right)^{2}+2 t\left\langle f\left(x_{t}\right)-x_{n}, J\left(x_{t}-x_{n}\right)\right\rangle \\
\leq & (1-t)^{2}\left(\left\|x_{t}-x_{n}\right\|+\left\|W x_{n}-x_{n}\right\|\right)^{2}+2 t\left\langle f\left(x_{t}\right)-x_{n}, J\left(x_{t}-x_{n}\right)\right\rangle \\
= & (1-t)^{2}\left[\left\|x_{t}-x_{n}\right\|^{2}+2\left\|x_{t}-x_{n}\right\|\left\|W x_{n}-x_{n}\right\|+\left\|W x_{n}-x_{n}\right\|^{2}\right] \\
& +2 t\left\langle f\left(x_{t}\right)-x_{t}, J\left(x_{t}-x_{n}\right)\right\rangle+2 t\left|x_{t}-x_{n}, J\left(x_{t}-x_{n}\right)\right\rangle \\
= & \left(1-2 t+t^{2}\right)\left\|x_{t}-x_{n}\right\|^{2}+f_{n}(t)+2 t\left\langle f\left(x_{t}\right)-x_{t}, J\left(x_{t}-x_{n}\right)\right\rangle+2 t\left\|x_{t}-x_{n}\right\|^{2},
\end{aligned}
$$

where

$$
f_{n}(t)=(1-t)^{2}\left(2\left\|x_{t}-x_{n}\right\|+\left\|x_{n}-W x_{n}\right\|\right)\left\|x_{n}-W x_{n}\right\| \rightarrow 0 \quad \text { as } n \rightarrow \infty .
$$

It follows from (3.18) that

$$
\left\langle x_{t}-f\left(x_{t}\right), J\left(x_{t}-x_{n}\right)\right\rangle \leq \frac{t}{2}\left\|x_{t}-x_{n}\right\|^{2}+\frac{1}{2 t} f_{n}(t) .
$$

Letting $n \rightarrow \infty$ in (3.20) and noticing (3.19), we derive

$$
\limsup _{n \rightarrow \infty}\left\langle x_{t}-f\left(x_{t}\right), J\left(x_{t}-x_{n}\right)\right\rangle \leq \frac{t}{2} M_{2}
$$

where $M_{2}>0$ is a constant such that $\left\|x_{t}-x_{n}\right\|^{2} \leq M_{2}$ for all $t \in(0,1)$ and $n \geq 0$. Taking $t \rightarrow 0$ in (3.21), we have

$$
\underset{t \rightarrow 0}{\limsup } \limsup _{n \rightarrow \infty}\left\langle x_{t}-f\left(x_{t}\right), J\left(x_{t}-x_{n}\right)\right\rangle \leq 0
$$


On the other hand, we have

$$
\begin{aligned}
\langle f(q) & \left.-q, J\left(x_{n}-q\right)\right\rangle \\
= & \left\langle f(q)-q, J\left(x_{n}-q\right)\right\rangle-\left\langle f(q)-q, J\left(x_{n}-x_{t}\right)\right\rangle+\left\langle f(q)-q, J\left(x_{n}-x_{t}\right)\right\rangle \\
& -\left\langle f(q)-x_{t}, J\left(x_{n}-x_{t}\right)\right\rangle+\left\langle f(q)-x_{t}, J\left(x_{n}-x_{t}\right)\right\rangle-\left\langle f\left(x_{t}\right)-x_{t}, J\left(x_{n}-x_{t}\right)\right\rangle \\
& +\left\langle f\left(x_{t}\right)-x_{t}, J\left(x_{n}-x_{t}\right)\right\rangle \\
= & \left\langle f(q)-q, J\left(x_{n}-q\right)-J\left(x_{n}-x_{t}\right)\right\rangle+\left\langle x_{t}-q, J\left(x_{n}-x_{t}\right)\right\rangle \\
& +\left\langle f(q)-f\left(x_{t}\right), J\left(x_{n}-x_{t}\right)\right\rangle+\left\langle f\left(x_{t}\right)-x_{t}, J\left(x_{n}-x_{t}\right)\right\rangle .
\end{aligned}
$$

It follows that

$$
\begin{aligned}
& \limsup _{n \rightarrow \infty}\left\langle f(q)-q, J\left(x_{n}-q\right)\right\rangle \\
& \leq \limsup _{n \rightarrow \infty}\left\langle f(q)-q, J\left(x_{n}-q\right)-J\left(x_{n}-x_{t}\right)\right\rangle \\
& \quad+\left\|x_{t}-q\right\| \limsup _{n \rightarrow \infty}\left\|x_{n}-x_{t}\right\|+\rho\left\|q-x_{t}\right\| \limsup _{n \rightarrow \infty}\left\|x_{n}-x_{t}\right\| \\
& \quad+\limsup _{n \rightarrow \infty}\left\langle f\left(x_{t}\right)-x_{t}, J\left(x_{n}-x_{t}\right)\right\rangle .
\end{aligned}
$$

Taking into account that $x_{t} \rightarrow q$ as $t \rightarrow 0$, we have from (3.22)

$$
\begin{aligned}
\limsup _{n \rightarrow \infty}\left\langle f(q)-q, J\left(x_{n}-q\right)\right\rangle & =\limsup _{t \rightarrow 0} \limsup _{n \rightarrow \infty}\left\langle f(q)-q, J\left(x_{n}-q\right)\right\rangle \\
& \leq \limsup _{t \rightarrow 0} \limsup _{n \rightarrow \infty}\left\langle f(q)-q, J\left(x_{n}-q\right)-J\left(x_{n}-x_{t}\right)\right\rangle .
\end{aligned}
$$

Since $X$ has a uniformly Gâteaux differentiable norm, the duality mapping $J$ is norm-toweak* uniformly continuous on bounded subsets of $X$. Consequently, the two limits are interchangeable and hence (3.17) holds. From (3.10) we get $\left(y_{n}-q\right)-\left(x_{n}-q\right) \rightarrow 0$. Noticing that $J$ is norm-to-weak* uniformly continuous on bounded subsets of $X$, we deduce from (3.17) that

$$
\begin{aligned}
\limsup _{n \rightarrow \infty}\left\langle f(q)-q, J\left(y_{n}-q\right)\right\rangle & =\limsup _{n \rightarrow \infty}\left\langle f(q)-q, J\left(x_{n}-q\right)\right\rangle \\
& \leq 0
\end{aligned}
$$

Finally, let us show that $x_{n} \rightarrow q$ as $n \rightarrow \infty$. We observe that

$$
\begin{aligned}
\| y_{n} & -q \|^{2} \\
& =\alpha_{n}\left\langle f\left(x_{n}\right)-q, J\left(y_{n}-q\right)\right\rangle+\left(1-\alpha_{n}\right)\left\langle G\left(x_{n}\right)-q, J\left(y_{n}-q\right)\right\rangle \\
& =\alpha_{n}\left\langle f\left(x_{n}\right)-f(q), J\left(y_{n}-q\right)\right\rangle+\alpha_{n}\left\langle f(q)-q, J\left(y_{n}-q\right)\right\rangle+\left(1-\alpha_{n}\right)\left\langle G\left(x_{n}\right)-q, J\left(y_{n}-q\right)\right\rangle \\
& \leq \alpha_{n} \rho\left\|x_{n}-q\right\|\left\|y_{n}-q\right\|+\left(1-\alpha_{n}\right)\left\|G\left(x_{n}\right)-q\right\|\left\|y_{n}-q\right\|+\alpha_{n}\left\langle f(q)-q, J\left(y_{n}-q\right)\right\rangle \\
& \leq \alpha_{n} \rho\left\|x_{n}-q\right\|\left\|y_{n}-q\right\|+\left(1-\alpha_{n}\right)\left\|x_{n}-q\right\|\left\|y_{n}-q\right\|+\alpha_{n}\left\langle f(q)-q, J\left(y_{n}-q\right)\right\rangle
\end{aligned}
$$




$$
\begin{aligned}
& =\left[1-\alpha_{n}(1-\rho)\right]\left\|x_{n}-q\right\|\left\|y_{n}-q\right\|+\alpha_{n}\left\langle f(q)-q, J\left(y_{n}-q\right)\right\rangle \\
& \leq \frac{1-\alpha_{n}(1-\rho)}{2}\left(\left\|x_{n}-q\right\|^{2}+\left\|y_{n}-q\right\|^{2}\right)+\alpha_{n}\left\langle f(q)-q, J\left(y_{n}-q\right)\right\rangle \\
& \leq \frac{1-\alpha_{n}(1-\rho)}{2}\left\|x_{n}-q\right\|^{2}+\frac{1}{2}\left\|y_{n}-q\right\|^{2}+\alpha_{n}\left\langle f(q)-q, J\left(y_{n}-q\right)\right\rangle,
\end{aligned}
$$

which implies that

$$
\left\|y_{n}-q\right\|^{2} \leq\left[1-\alpha_{n}(1-\rho)\right]\left\|x_{n}-q\right\|^{2}+\alpha_{n}(1-\rho) \frac{2\left\langle f(q)-q, J\left(y_{n}-q\right)\right\rangle}{1-\rho} .
$$

Also, by the convexity of $\|\cdot\|^{2}$ and (3.1), we get

$$
\left\|x_{n+1}-q\right\|^{2} \leq \beta_{n}\left\|x_{n}-q\right\|^{2}+\gamma_{n}\left\|y_{n}-q\right\|^{2}+\delta_{n}\left\|x_{n+1}-q\right\|^{2},
$$

which together with (3.24) leads to

$$
\begin{aligned}
\left\|x_{n+1}-q\right\|^{2} \leq & \frac{\beta_{n}}{\beta_{n}+\gamma_{n}}\left\|x_{n}-q\right\|^{2}+\frac{\gamma_{n}}{\beta_{n}+\gamma_{n}}\left\|y_{n}-q\right\|^{2} \\
\leq & \frac{\beta_{n}}{\beta_{n}+\gamma_{n}}\left\|x_{n}-q\right\|^{2}+\frac{\gamma_{n}}{\beta_{n}+\gamma_{n}}\left[\left(1-\alpha_{n}(1-\rho)\right)\left\|x_{n}-q\right\|^{2}\right. \\
& \left.+\alpha_{n}(1-\rho) \frac{2\left\langle f(q)-q, J\left(y_{n}-q\right)\right\rangle}{1-\rho}\right] \\
= & {\left[1-\frac{\alpha_{n} \gamma_{n}(1-\rho)}{\beta_{n}+\gamma_{n}}\right]\left\|x_{n}-q\right\|^{2} } \\
& +\frac{\alpha_{n} \gamma_{n}(1-\rho)}{\beta_{n}+\gamma_{n}} \cdot \frac{2\left\langle f(q)-q, J\left(y_{n}-q\right)\right\rangle}{1-\rho} .
\end{aligned}
$$

Applying Lemma 2.1 to (3.25), we obtain that $x_{n} \rightarrow q$ as $n \rightarrow \infty$. This completes the proof.

Corollary 3.1 Let $C$ be a nonempty closed convex subset of a uniformly convex Banach space $X$ which has a uniformly Gâteaux differentiable norm. Let $\Pi_{C}$ be a sunny nonexpansive retraction from $X$ onto $C$. Let the mapping $B_{i}: C \rightarrow X$ be $\lambda_{i}$-strictly pseudocontractive and $\alpha_{i}$-strongly accretive with $\alpha_{i}+\lambda_{i} \geq 1$ for $i=1,2$. Let $f: C \rightarrow C$ be a contraction with coefficient $\rho \in(0,1)$. Let $S$ be a nonexpansive mapping of $C$ into itself such that $F=$ $\operatorname{Fix}(S) \cap \Omega \neq \emptyset$, where $\Omega$ is the fixed point set of the mapping $G=\Pi_{C}\left(I-\mu_{1} B_{1}\right) \Pi_{C}\left(I-\mu_{2} B_{2}\right)$. For arbitrarily given $x_{0} \in C$, let $\left\{x_{n}\right\}$ be the sequence generated by

$$
\left\{\begin{array}{l}
y_{n}=\alpha_{n} f\left(x_{n}\right)+\left(1-\alpha_{n}\right) G\left(x_{n}\right), \\
x_{n+1}=\beta_{n} x_{n}+\gamma_{n} y_{n}+\delta_{n} S x_{n+1}, \quad \forall n \geq 0,
\end{array}\right.
$$

where $1-\frac{\lambda_{i}}{1+\lambda_{i}}\left(1-\sqrt{\frac{1-\alpha_{i}}{\lambda_{i}}}\right) \leq \mu_{i} \leq 1$ for $i=1,2$. Suppose that $\left\{\alpha_{n}\right\},\left\{\beta_{n}\right\},\left\{\gamma_{n}\right\}$ and $\left\{\delta_{n}\right\}$ are the sequences in $(0,1)$ satisfying the following conditions:

(i) $\lim _{n \rightarrow \infty} \alpha_{n}=0$ and $\sum_{n=0}^{\infty} \alpha_{n}=\infty$;

(ii) $\beta_{n}+\gamma_{n}+\delta_{n}=1, \forall n \geq 0$, and $\liminf _{n \rightarrow \infty} \delta_{n}>0$;

(iii) $0<\liminf _{n \rightarrow \infty} \beta_{n} \leq \limsup _{n \rightarrow \infty}\left(\beta_{n}+\delta_{n}\right)<1$; 
(iv) $\sum_{n=1}^{\infty}\left|\frac{\gamma_{n}}{1-\beta_{n}}-\frac{\gamma_{n-1}}{1-\beta_{n-1}}\right|<\infty$ or $\lim _{n \rightarrow \infty} \frac{1}{\alpha_{n}}\left|\frac{\gamma_{n}}{1-\beta_{n}}-\frac{\gamma_{n-1}}{1-\beta_{n-1}}\right|=0$;

(v) $\sum_{n=1}^{\infty}\left|\alpha_{n}-\alpha_{n-1}\right|<\infty$ or $\lim _{n \rightarrow \infty} \alpha_{n-1} / \alpha_{n}=1$;

(vi) $\sum_{n=1}^{\infty}\left|\beta_{n}-\beta_{n-1}\right|<\infty$ or $\lim _{n \rightarrow \infty}\left|\beta_{n}-\beta_{n-1}\right| / \alpha_{n}=0$.

Then $\left\{x_{n}\right\}$ converges strongly to $q \in F$, which solves the following VIP:

$$
\langle q-f(q), J(q-p)\rangle \leq 0, \quad \forall p \in F
$$

\section{Three-step implicit iterative algorithm}

In this section, we introduce our three-step implicit iterative algorithm and show strong convergence of the purposed algorithm.

Theorem 4.1 Let $C$ be a nonempty closed convex subset of a uniformly convex Banach space $X$ which has a uniformly Gâteaux differentiable norm. Let $\Pi_{C}$ be a sunny nonexpansive retraction from $X$ onto $C$. Let the mapping $B_{i}: C \rightarrow X$ be $\lambda_{i}$-strictly pseudocontractive and $\alpha_{i}$-strongly accretive with $\alpha_{i}+\lambda_{i} \geq 1$ for $i=1,2$. Let $f: C \rightarrow C$ be a contraction with coefficient $\rho \in(0,1)$. Let $\left\{S_{n}\right\}_{n=1}^{\infty}$ be an infinite family of nonexpansive mappings of $C$ into itself such that $F=\bigcap_{i=1}^{\infty} \operatorname{Fix}\left(S_{i}\right) \cap \Omega \neq \emptyset$, where $\Omega$ is the fixed point set of the mapping $G=\Pi_{C}\left(I-\mu_{1} B_{1}\right) \Pi_{C}\left(I-\mu_{2} B_{2}\right)$. For arbitrarily given $x_{0} \in C$, let $\left\{x_{n}\right\}$ be the sequence generated by

$$
\left\{\begin{array}{l}
z_{n}=\sigma_{n} G\left(x_{n}\right)+\left(1-\sigma_{n}\right) S_{n} G\left(x_{n}\right), \\
y_{n}=\alpha_{n} f\left(x_{n}\right)+\left(1-\alpha_{n}\right) z_{n}, \\
x_{n+1}=\beta_{n} x_{n}+\gamma_{n} y_{n}+\delta_{n} S_{n+1} G\left(x_{n+1}\right), \quad \forall n \geq 0,
\end{array}\right.
$$

where $1-\frac{\lambda_{i}}{1+\lambda_{i}}\left(1-\sqrt{\frac{1-\alpha_{i}}{\lambda_{i}}}\right) \leq \mu_{i} \leq 1$ for $i=1,2$. Suppose that $\left\{\sigma_{n}\right\},\left\{\alpha_{n}\right\},\left\{\beta_{n}\right\},\left\{\gamma_{n}\right\}$ and $\left\{\delta_{n}\right\}$ are the sequences in $(0,1)$ satisfying the following conditions:

(i) $0<\liminf _{n \rightarrow \infty} \sigma_{n} \leq \limsup _{n \rightarrow \infty} \sigma_{n}<1$;

(ii) $\lim _{n \rightarrow \infty} \alpha_{n}=0$ and $\sum_{n=0}^{\infty} \alpha_{n}=\infty$;

(iii) $\beta_{n}+\gamma_{n}+\delta_{n}=1, \forall n \geq 0$, and $\liminf _{n \rightarrow \infty} \delta_{n}>0$;

(iv) $0<\liminf _{n \rightarrow \infty} \beta_{n} \leq \limsup _{n \rightarrow \infty}\left(\beta_{n}+\delta_{n}\right)<1$;

(v) $\sum_{n=1}^{\infty}\left|\frac{\gamma_{n}}{1-\beta_{n}}-\frac{\gamma_{n-1}}{1-\beta_{n-1}}\right|<\infty$ or $\lim _{n \rightarrow \infty} \frac{1}{\alpha_{n}}\left|\frac{\gamma_{n}}{1-\beta_{n}}-\frac{\gamma_{n-1}}{1-\beta_{n-1}}\right|=0$;

(vi) $\sum_{n=1}^{\infty}\left|\alpha_{n}-\alpha_{n-1}\right|<\infty$ or $\lim _{n \rightarrow \infty} \alpha_{n-1} / \alpha_{n}=1$;

(vii) $\sum_{n=1}^{\infty}\left|\beta_{n}-\beta_{n-1}\right|<\infty$ or $\lim _{n \rightarrow \infty}\left|\beta_{n}-\beta_{n-1}\right| / \alpha_{n}=0$;

(viii) $\sum_{n=1}^{\infty}\left|\sigma_{n}-\sigma_{n-1}\right|<\infty$ or $\lim _{n \rightarrow \infty}\left|\sigma_{n}-\sigma_{n-1}\right| / \alpha_{n}=0$.

Assume that $\sum_{n=1}^{\infty} \sup _{x \in D}\left\|S_{n} x-S_{n-1} x\right\|<\infty$ for any bounded subset $D$ of $C$, and let $S$ be a mapping of $C$ into itself defined by $S x=\lim _{n \rightarrow \infty} S_{n} x$ for all $x \in C$ and suppose that $\operatorname{Fix}(S)=$ $\bigcap_{i=1}^{\infty} \operatorname{Fix}\left(S_{i}\right)$. Then $\left\{x_{n}\right\}$ converges strongly to $q \in F$, which solves the following VIP:

$$
\langle q-f(q), J(q-p)\rangle \leq 0, \quad \forall p \in F
$$

Proof Take a fixed $p \in F$ arbitrarily. Then by Lemma 2.11 we know that $p=G(p)$ and $p=$ $S_{n} p$ for all $n \geq 1$. Moreover, by Lemma 2.10 we have

$$
\begin{aligned}
\left\|z_{n}-p\right\| & =\left\|\sigma_{n}\left(G\left(x_{n}\right)-p\right)+\left(1-\sigma_{n}\right)\left(S_{n} G\left(x_{n}\right)-p\right)\right\| \\
& \leq \sigma_{n}\left\|G\left(x_{n}\right)-p\right\|+\left(1-\sigma_{n}\right)\left\|S_{n} G\left(x_{n}\right)-p\right\|
\end{aligned}
$$




$$
\begin{aligned}
& \leq \sigma_{n}\left\|G\left(x_{n}\right)-p\right\|+\left(1-\sigma_{n}\right)\left\|G\left(x_{n}\right)-p\right\| \\
& =\left\|G\left(x_{n}\right)-p\right\| \\
& \leq\left\|x_{n}-p\right\|,
\end{aligned}
$$

and

$$
\begin{aligned}
\left\|y_{n}-p\right\| & =\left\|\alpha_{n}\left(f\left(x_{n}\right)-p\right)+\left(1-\alpha_{n}\right)\left(z_{n}-p\right)\right\| \\
& \leq \alpha_{n}\left\|f\left(x_{n}\right)-f(p)\right\|+\alpha_{n}\|f(p)-p\|+\left(1-\alpha_{n}\right)\left\|z_{n}-p\right\| \\
& \leq \alpha_{n} \rho\left\|x_{n}-p\right\|+\alpha_{n}\|f(p)-p\|+\left(1-\alpha_{n}\right)\left\|x_{n}-p\right\| \\
& =\left(1-\alpha_{n}(1-\rho)\right)\left\|x_{n}-p\right\|+\alpha_{n}\|f(p)-p\| .
\end{aligned}
$$

From (4.1) we obtain

$$
\begin{aligned}
\left\|x_{n+1}-p\right\| & =\left\|\beta_{n}\left(x_{n}-p\right)+\gamma_{n}\left(y_{n}-p\right)+\delta_{n}\left(S_{n+1} G\left(x_{n+1}\right)-p\right)\right\| \\
& \leq \beta_{n}\left\|x_{n}-p\right\|+\gamma_{n}\left\|y_{n}-p\right\|+\delta_{n}\left\|x_{n+1}-p\right\|,
\end{aligned}
$$

which together with (4.4) implies that

$$
\begin{aligned}
\left\|x_{n+1}-p\right\| & \leq \frac{1}{\beta_{n}+\gamma_{n}}\left[\beta_{n}\left\|x_{n}-p\right\|+\gamma_{n}\left\|y_{n}-p\right\|\right] \\
& \leq \frac{1}{\beta_{n}+\gamma_{n}}\left\{\beta_{n}\left\|x_{n}-p\right\|+\gamma_{n}\left[\left(1-\alpha_{n}(1-\rho)\right)\left\|x_{n}-p\right\|+\alpha_{n}\|f(p)-p\|\right]\right\} \\
& =\frac{1}{\beta_{n}+\gamma_{n}}\left\{\left(\beta_{n}+\gamma_{n}-\alpha_{n} \gamma_{n}(1-\rho)\right)\left\|x_{n}-p\right\|+\alpha_{n} \gamma_{n}\|f(p)-p\|\right\} \\
& =\left(1-\frac{\alpha_{n} \gamma_{n}(1-\rho)}{\beta_{n}+\gamma_{n}}\right)\left\|x_{n}-p\right\|+\frac{\alpha_{n} \gamma_{n}}{\beta_{n}+\gamma_{n}}\|f(p)-p\| \\
& =\left(1-\frac{\alpha_{n} \gamma_{n}(1-\rho)}{\beta_{n}+\gamma_{n}}\right)\left\|x_{n}-p\right\|+\frac{\alpha_{n} \gamma_{n}(1-\rho)}{\beta_{n}+\gamma_{n}} \cdot \frac{\|f(p)-p\|}{1-\rho} \\
& \leq \max \left\{\left\|x_{0}-p\right\|, \frac{\|f(p)-p\|}{1-\rho}\right\}
\end{aligned}
$$

which implies that $\left\{x_{n}\right\}$ is bounded. By Lemma 2.10 we know from (4.3) and (4.4) that $\left\{y_{n}\right\}$, $\left\{z_{n}\right\},\left\{G\left(x_{n}\right)\right\}$ and $\left\{G\left(y_{n}\right)\right\}$ are bounded.

Let us show that $\left\|x_{n+1}-x_{n}\right\| \rightarrow 0$ and $\left\|x_{n}-y_{n}\right\| \rightarrow 0$ as $n \rightarrow \infty$. As a matter of fact, from (4.1) we have

$$
\begin{aligned}
z_{n}-z_{n-1}= & \sigma_{n}\left(G\left(x_{n}\right)-G\left(x_{n-1}\right)\right)+\left(\sigma_{n}-\sigma_{n-1}\right)\left(G\left(x_{n-1}\right)-S_{n-1} G\left(x_{n-1}\right)\right) \\
& +\left(1-\sigma_{n}\right)\left(S_{n} G\left(x_{n}\right)-S_{n-1} G\left(x_{n-1}\right)\right),
\end{aligned}
$$

and

$$
\begin{aligned}
y_{n}-y_{n-1}= & \alpha_{n}\left(f\left(x_{n}\right)-f\left(x_{n-1}\right)\right)+\left(\alpha_{n}-\alpha_{n-1}\right)\left(f\left(x_{n-1}\right)-z_{n-1}\right) \\
& +\left(1-\alpha_{n}\right)\left(z_{n}-z_{n-1}\right) .
\end{aligned}
$$


It follows that

$$
\begin{aligned}
\left\|z_{n}-z_{n-1}\right\| \leq & \sigma_{n}\left\|G\left(x_{n}\right)-G\left(x_{n-1}\right)\right\|+\left|\sigma_{n}-\sigma_{n-1}\right|\left\|G\left(x_{n-1}\right)-S_{n-1} G\left(x_{n-1}\right)\right\| \\
& +\left(1-\sigma_{n}\right)\left\|S_{n} G\left(x_{n}\right)-S_{n-1} G\left(x_{n-1}\right)\right\| \\
\leq & \sigma_{n}\left\|G\left(x_{n}\right)-G\left(x_{n-1}\right)\right\|+\left|\sigma_{n}-\sigma_{n-1}\right|\left\|G\left(x_{n-1}\right)-S_{n-1} G\left(x_{n-1}\right)\right\| \\
& +\left(1-\sigma_{n}\right)\left(\left\|S_{n} G\left(x_{n}\right)-S_{n} G\left(x_{n-1}\right)\right\|+\left\|S_{n} G\left(x_{n-1}\right)-S_{n-1} G\left(x_{n-1}\right)\right\|\right) \\
\leq & \sigma_{n}\left\|G\left(x_{n}\right)-G\left(x_{n-1}\right)\right\|+\left|\sigma_{n}-\sigma_{n-1}\right|\left\|G\left(x_{n-1}\right)-S_{n-1} G\left(x_{n-1}\right)\right\| \\
& +\left(1-\sigma_{n}\right)\left(\left\|G\left(x_{n}\right)-G\left(x_{n-1}\right)\right\|+\left\|S_{n} G\left(x_{n-1}\right)-S_{n-1} G\left(x_{n-1}\right)\right\|\right) \\
\leq & \left\|G\left(x_{n}\right)-G\left(x_{n-1}\right)\right\|+\left|\sigma_{n}-\sigma_{n-1}\right|\left\|G\left(x_{n-1}\right)-S_{n-1} G\left(x_{n-1}\right)\right\| \\
& +\left\|S_{n} G\left(x_{n-1}\right)-S_{n-1} G\left(x_{n-1}\right)\right\| \\
\leq & \left\|x_{n}-x_{n-1}\right\|+\left|\sigma_{n}-\sigma_{n-1}\right|\left\|G\left(x_{n-1}\right)-S_{n-1} G\left(x_{n-1}\right)\right\| \\
& +\left\|S_{n} G\left(x_{n-1}\right)-S_{n-1} G\left(x_{n-1}\right)\right\|,
\end{aligned}
$$

and

$$
\begin{aligned}
\left\|y_{n}-y_{n-1}\right\| \leq & \alpha_{n}\left\|f\left(x_{n}\right)-f\left(x_{n-1}\right)\right\|+\left|\alpha_{n}-\alpha_{n-1}\right|\left\|f\left(x_{n-1}\right)-z_{n-1}\right\| \\
& +\left(1-\alpha_{n}\right)\left\|z_{n}-z_{n-1}\right\| \\
\leq & \alpha_{n} \rho\left\|x_{n}-x_{n-1}\right\|+\left|\alpha_{n}-\alpha_{n-1}\right|\left\|f\left(x_{n-1}\right)-z_{n-1}\right\| \\
& +\left(1-\alpha_{n}\right)\left\|z_{n}-z_{n-1}\right\| \\
\leq & \alpha_{n} \rho\left\|x_{n}-x_{n-1}\right\|+\left|\alpha_{n}-\alpha_{n-1}\right|\left\|f\left(x_{n-1}\right)-z_{n-1}\right\| \\
& +\left(1-\alpha_{n}\right)\left[\left\|x_{n}-x_{n-1}\right\|+\left|\sigma_{n}-\sigma_{n-1}\right|\left\|G\left(x_{n-1}\right)-S_{n-1} G\left(x_{n-1}\right)\right\|\right. \\
& \left.+\left\|S_{n} G\left(x_{n-1}\right)-S_{n-1} G\left(x_{n-1}\right)\right\|\right] \\
\leq & \left(1-\alpha_{n}(1-\rho)\right)\left\|x_{n}-x_{n-1}\right\|+\left|\sigma_{n}-\sigma_{n-1}\right|\left\|G\left(x_{n-1}\right)-S_{n-1} G\left(x_{n-1}\right)\right\| \\
& +\left|\alpha_{n}-\alpha_{n-1}\right|\left\|f\left(x_{n-1}\right)-z_{n-1}\right\|+\left\|S_{n} G\left(x_{n-1}\right)-S_{n-1} G\left(x_{n-1}\right)\right\| .
\end{aligned}
$$

Taking into consideration that $0<\liminf _{n \rightarrow \infty} \beta_{n} \leq \limsup _{n \rightarrow \infty}\left(\beta_{n}+\delta_{n}\right)<1$ and $\liminf _{n \rightarrow \infty} \gamma_{n}=1-\limsup _{n \rightarrow \infty}\left(\beta_{n}+\delta_{n}\right)>0$, we may assume that $\left\{\beta_{n}\right\} \subset[c, d]$ and $\left\{\gamma_{n}\right\} \subset$ $[c, 1)$ for some $c, d \in(0,1)$. Now, we write $x_{n}=\beta_{n-1} x_{n-1}+\left(1-\beta_{n-1}\right) v_{n-1}, \forall n \geq 1$, where $v_{n-1}=\frac{x_{n}-\beta_{n-1} x_{n-1}}{1-\beta_{n-1}}$. It follows that for all $n \geq 1$,

$$
\begin{aligned}
v_{n}-v_{n-1}= & \frac{x_{n+1}-\beta_{n} x_{n}}{1-\beta_{n}}-\frac{x_{n}-\beta_{n-1} x_{n-1}}{1-\beta_{n-1}} \\
= & \frac{\gamma_{n} y_{n}+\delta_{n} S_{n+1} G\left(x_{n+1}\right)}{1-\beta_{n}}-\frac{\gamma_{n-1} y_{n-1}+\delta_{n-1} S_{n} G\left(x_{n}\right)}{1-\beta_{n-1}} \\
= & \frac{\gamma_{n}\left(y_{n}-y_{n-1}\right)+\delta_{n}\left(S_{n+1} G\left(x_{n+1}\right)-S_{n} G\left(x_{n}\right)\right)}{1-\beta_{n}} \\
& +\left(\frac{\gamma_{n}}{1-\beta_{n}}-\frac{\gamma_{n-1}}{1-\beta_{n-1}}\right) y_{n-1}+\left(\frac{\delta_{n}}{1-\beta_{n}}-\frac{\delta_{n-1}}{1-\beta_{n-1}}\right) S_{n} G\left(x_{n}\right) .
\end{aligned}
$$


This together with (4.5) implies that

$$
\begin{aligned}
\| v_{n}- & v_{n-1} \| \\
\leq & \frac{\left\|\gamma_{n}\left(y_{n}-y_{n-1}\right)+\delta_{n}\left(S_{n+1} G\left(x_{n+1}\right)-S_{n} G\left(x_{n}\right)\right)\right\|}{1-\beta_{n}}+\left|\frac{\gamma_{n}}{1-\beta_{n}}-\frac{\gamma_{n-1}}{1-\beta_{n-1}}\right|\left\|y_{n-1}\right\| \\
& +\left|\frac{\delta_{n}}{1-\beta_{n}}-\frac{\delta_{n-1}}{1-\beta_{n-1}}\right|\left\|S_{n} G\left(x_{n}\right)\right\| \\
\leq & \frac{\gamma_{n}\left\|y_{n}-y_{n-1}\right\|+\delta_{n}\left(\left\|S_{n+1} G\left(x_{n+1}\right)-S_{n+1} G\left(x_{n}\right)\right\|+\left\|S_{n+1} G\left(x_{n}\right)-S_{n} G\left(x_{n}\right)\right\|\right)}{1-\beta_{n}} \\
& +\left|\frac{\gamma_{n}}{1-\beta_{n}}-\frac{\gamma_{n-1}}{1-\beta_{n-1}}\right|\left\|y_{n-1}\right\|+\left|\frac{\gamma_{n}}{1-\beta_{n}}-\frac{\gamma_{n-1}}{1-\beta_{n-1}}\right|\left\|S_{n} G\left(x_{n}\right)\right\| \\
\leq & \frac{\gamma_{n}\left\|y_{n}-y_{n-1}\right\|+\delta_{n}\left\|x_{n+1}-x_{n}\right\|}{1-\beta_{n}}+\frac{\delta_{n}}{1-\beta_{n}}\left\|S_{n+1} G\left(x_{n}\right)-S_{n} G\left(x_{n}\right)\right\| \\
& +\left|\frac{\gamma_{n}}{1-\beta_{n}}-\frac{\gamma_{n-1}}{1-\beta_{n-1}}\right|\left(\left\|y_{n-1}\right\|+\left\|S_{n} G\left(x_{n}\right)\right\|\right) \\
\leq & \frac{\gamma_{n}}{\gamma_{n}+\delta_{n}}\left[\left(1-\alpha_{n}(1-\rho)\right)\left\|x_{n}-x_{n-1}\right\|+\left|\sigma_{n}-\sigma_{n-1}\right|\left\|G\left(x_{n-1}\right)-S_{n-1} G\left(x_{n-1}\right)\right\|\right. \\
& \left.+\left|\alpha_{n}-\alpha_{n-1}\right|\left\|f\left(x_{n-1}\right)-z_{n-1}\right\|+\left\|S_{n} G\left(x_{n-1}\right)-S_{n-1} G\left(x_{n-1}\right)\right\|\right] \\
& +\frac{\delta_{n}}{\gamma_{n}+\delta_{n}}\left\|x_{n+1}-x_{n}\right\|+\frac{\delta_{n}}{\gamma_{n}+\delta_{n}}\left\|S_{n+1} G\left(x_{n}\right)-S_{n} G\left(x_{n}\right)\right\| \\
& +\left|\frac{\gamma_{n}}{1-\beta_{n}}-\frac{\gamma_{n-1}}{1-\beta_{n-1}}\right|\left(\left\|y_{n-1}\right\|+\left\|S_{n} G\left(x_{n}\right)\right\|\right) \\
\leq & \frac{\gamma_{n}}{\gamma_{n}+\delta_{n}}\left[\left(1-\alpha_{n}(1-\rho)\right)\left\|x_{n}-x_{n-1}\right\|+\left|\sigma_{n}-\sigma_{n-1}\right| M\right. \\
& \left.+\left|\alpha_{n}-\alpha_{n-1}\right| M+\left\|S_{n} G\left(x_{n-1}\right)-S_{n-1} G\left(x_{n-1}\right)\right\|\right]+\frac{\delta_{n}}{\gamma_{n}+\delta_{n}}\left\|x_{n+1}-x_{n}\right\| \\
& +\frac{\delta_{n}}{\gamma_{n}+\delta_{n}}\left\|S_{n+1} G\left(x_{n}\right)-S_{n} G\left(x_{n}\right)\right\|+\left|\frac{\gamma_{n}}{1-\beta_{n}}-\frac{\gamma_{n-1}}{1-\beta_{n-1}}\right| M, \\
&
\end{aligned}
$$

where $\sup _{n \geq 0}\left\{\left\|f\left(x_{n}\right)-z_{n}\right\|+\left\|G\left(x_{n}\right)-S_{n} G\left(x_{n}\right)\right\|\right\} \leq M$ and $\sup _{n \geq 1}\left\{\left\|y_{n-1}\right\|+\left\|S_{n} x_{n}\right\|\right\} \leq M$ for some $M>0$.

Now we observe that

$$
\begin{aligned}
& x_{n+1}-x_{n}=\left(\beta_{n}-\beta_{n-1}\right)\left(x_{n}-v_{n}\right)+\beta_{n-1}\left(x_{n}-x_{n-1}\right)+\left(1-\beta_{n-1}\right)\left(v_{n}-v_{n-1}\right), \\
& 1-\left(1-\beta_{n-1}\right) \frac{\delta_{n}}{\gamma_{n}+\delta_{n}}=\frac{\gamma_{n}+\beta_{n-1} \delta_{n}}{\gamma_{n}+\delta_{n}},
\end{aligned}
$$

and

$$
\begin{aligned}
\beta_{n-1}+\left(1-\beta_{n-1}\right) \frac{\gamma_{n}}{\gamma_{n}+\delta_{n}}\left(1-(1-\rho) \alpha_{n}\right) \\
=\beta_{n-1}\left(\frac{\gamma_{n}}{\gamma_{n}+\delta_{n}}+\frac{\delta_{n}}{\gamma_{n}+\delta_{n}}\right)+\left(1-\beta_{n-1}\right) \frac{\gamma_{n}}{\gamma_{n}+\delta_{n}}\left(1-(1-\rho) \alpha_{n}\right) \\
=\frac{\beta_{n-1} \delta_{n}}{\gamma_{n}+\delta_{n}}+\frac{\gamma_{n}}{\gamma_{n}+\delta_{n}}\left[\beta_{n-1}+\left(1-\beta_{n-1}\right)\left(1-(1-\rho) \alpha_{n}\right)\right]
\end{aligned}
$$




$$
\begin{aligned}
& =\frac{\beta_{n-1} \delta_{n}}{\gamma_{n}+\delta_{n}}+\frac{\gamma_{n}}{\gamma_{n}+\delta_{n}}\left[1-\left(1-\beta_{n-1}\right)(1-\rho) \alpha_{n}\right] \\
& =\frac{\gamma_{n}+\beta_{n-1} \delta_{n}}{\gamma_{n}+\delta_{n}}-\left(1-\beta_{n-1}\right)(1-\rho) \frac{\alpha_{n} \gamma_{n}}{\gamma_{n}+\delta_{n}} .
\end{aligned}
$$

Hence from (4.7) it follows that

$$
\begin{aligned}
&\left\|x_{n+1}-x_{n}\right\| \\
& \leq\left|\beta_{n}-\beta_{n-1}\right|\left\|x_{n}-v_{n}\right\|+\beta_{n-1}\left\|x_{n}-x_{n-1}\right\|+\left(1-\beta_{n-1}\right)\left\|v_{n}-v_{n-1}\right\| \\
& \leq\left|\beta_{n}-\beta_{n-1}\right|\left\|x_{n}-v_{n}\right\|+\beta_{n-1}\left\|x_{n}-x_{n-1}\right\| \\
&+\left(1-\beta_{n-1}\right)\left\{\frac { \gamma _ { n } } { \gamma _ { n } + \delta _ { n } } \left[\left(1-\alpha_{n}(1-\rho)\right)\left\|x_{n}-x_{n-1}\right\|+\left|\sigma_{n}-\sigma_{n-1}\right| M\right.\right. \\
&\left.+\left|\alpha_{n}-\alpha_{n-1}\right| M+\left\|S_{n} G\left(x_{n-1}\right)-S_{n-1} G\left(x_{n-1}\right)\right\|\right]+\frac{\delta_{n}}{\gamma_{n}+\delta_{n}}\left\|x_{n+1}-x_{n}\right\| \\
&+\frac{\delta_{n}}{\gamma_{n}+\delta_{n}}\left\|S_{n+1} G\left(x_{n}\right)-S_{n} G\left(x_{n}\right)\right\|+\mid \frac{\gamma_{n}}{1-\beta_{n}}-\frac{\gamma_{n-1}}{\left.1-\beta_{n-1} \mid M\right\}} \\
& \leq\left|\beta_{n}-\beta_{n-1}\right| M_{1}+\left[\beta_{n-1}+\left(1-\beta_{n-1}\right) \frac{\gamma_{n}}{\gamma_{n}+\delta_{n}}\left(1-(1-\rho) \alpha_{n}\right)\right]\left\|x_{n}-x_{n-1}\right\| \\
&+\left|\sigma_{n}-\sigma_{n-1}\right| M_{1}+\left|\alpha_{n}-\alpha_{n-1}\right| M_{1}+\left\|S_{n} G\left(x_{n-1}\right)-S_{n-1} G\left(x_{n-1}\right)\right\| \\
&+\left(1-\beta_{n-1}\right) \frac{\delta_{n}}{\gamma_{n}+\delta_{n}}\left\|x_{n+1}-x_{n}\right\|+\left\|S_{n+1} G\left(x_{n}\right)-S_{n} G\left(x_{n}\right)\right\|+\left|\frac{\gamma_{n}}{1-\beta_{n}}-\frac{\gamma_{n-1}}{1-\beta_{n-1}}\right| M_{1} \\
&= {\left[\frac{\gamma_{n}+\beta_{n-1} \delta_{n}}{\gamma_{n}+\delta_{n}}-\left(1-\beta_{n-1}\right)(1-\rho) \frac{\alpha_{n} \gamma_{n}}{\gamma_{n}+\delta_{n}}\right]\left\|x_{n}-x_{n-1}\right\| } \\
&+\left(1-\beta_{n-1}\right) \frac{\delta_{n}}{\gamma_{n}+\delta_{n}}\left\|x_{n+1}-x_{n}\right\| \\
&+M_{1}\left(\left|\sigma_{n}-\sigma_{n-1}\right|+\left|\alpha_{n}-\alpha_{n-1}\right|+\left|\beta_{n}-\beta_{n-1}\right|+\left|\frac{\gamma_{n}}{1-\beta_{n}}-\frac{\gamma_{n-1}}{1-\beta_{n-1}}\right|\right) \\
&+\left\|S_{n} G\left(x_{n-1}\right)-S_{n-1} G\left(x_{n-1}\right)\right\|+\left\|S_{n+1} G\left(x_{n}\right)-S_{n} G\left(x_{n}\right)\right\|, \\
&
\end{aligned}
$$

where $\sup _{n \geq 0}\left\{M+\left\|x_{n}-v_{n}\right\|\right\} \leq M_{1}$ for some $M_{1}>0$. Therefore, we get

$$
\begin{aligned}
&\left\|x_{n+1}-x_{n}\right\| \\
& \leq {\left[1-\left(1-\beta_{n-1}\right)(1-\rho) \frac{\alpha_{n} \gamma_{n}}{\gamma_{n}+\beta_{n-1} \delta_{n}}\right]\left\|x_{n}-x_{n-1}\right\|+\frac{\gamma_{n}+\delta_{n}}{\gamma_{n}+\beta_{n-1} \delta_{n}}\left[M _ { 1 } \left(\left|\sigma_{n}-\sigma_{n-1}\right|\right.\right.} \\
&\left.+\left|\alpha_{n}-\alpha_{n-1}\right|+\left|\beta_{n}-\beta_{n-1}\right|+\left|\frac{\gamma_{n}}{1-\beta_{n}}-\frac{\gamma_{n-1}}{1-\beta_{n-1}}\right|\right) \\
&\left.+\left\|S_{n} G\left(x_{n-1}\right)-S_{n-1} G\left(x_{n-1}\right)\right\|+\left\|S_{n+1} G\left(x_{n}\right)-S_{n} G\left(x_{n}\right)\right\|\right] \\
& \leq {\left[1-c(1-d)(1-\rho) \frac{\alpha_{n}}{\gamma_{n}+\beta_{n-1} \delta_{n}}\right]\left\|x_{n}-x_{n-1}\right\| } \\
&+\frac{1}{\gamma_{n}+\beta_{n-1} \delta_{n}}\left[M_{1}\left(\left|\alpha_{n}-\alpha_{n-1}\right|+\left|\beta_{n}-\beta_{n-1}\right|+\left|\frac{\gamma_{n}}{1-\beta_{n}}-\frac{\gamma_{n-1}}{1-\beta_{n-1}}\right|\right)\right.
\end{aligned}
$$




$$
\begin{aligned}
& \left.+\left\|S_{n+1} x_{n}-S_{n} x_{n}\right\|\right] \\
= & {\left[1-c(1-d)(1-\rho) \frac{\alpha_{n}}{\gamma_{n}+\beta_{n-1} \delta_{n}}\right]\left\|x_{n}-x_{n-1}\right\| } \\
& +c(1-d)(1-\rho) \frac{1}{\gamma_{n}+\beta_{n-1} \delta_{n}} \cdot \frac{1}{c(1-d)(1-\rho)}\left[M _ { 1 } \left(\left|\sigma_{n}-\sigma_{n-1}\right|+\left|\alpha_{n}-\alpha_{n-1}\right|\right.\right. \\
& \left.+\left|\beta_{n}-\beta_{n-1}\right|+\left|\frac{\gamma_{n}}{1-\beta_{n}}-\frac{\gamma_{n-1}}{1-\beta_{n-1}}\right|\right)+\left\|S_{n} G\left(x_{n-1}\right)-S_{n-1} G\left(x_{n-1}\right)\right\| \\
& \left.+\left\|S_{n+1} G\left(x_{n}\right)-S_{n} G\left(x_{n}\right)\right\|\right] \\
\leq & {\left[1-c(1-d)(1-\rho) \frac{\alpha_{n}}{\gamma_{n}+\beta_{n-1} \delta_{n}}\right]\left\|x_{n}-x_{n-1}\right\| } \\
& +\frac{1}{\gamma_{n}+\beta_{n-1} \delta_{n}}\left[M_{1}\left(\left|\alpha_{n}-\alpha_{n-1}\right|+\left|\beta_{n}-\beta_{n-1}\right|+\left|\frac{\gamma_{n}}{1-\beta_{n}}-\frac{\gamma_{n-1}}{1-\beta_{n-1}}\right|\right)\right. \\
& \left.+\left\|S_{n+1} x_{n}-S_{n} x_{n}\right\|\right] .
\end{aligned}
$$

Utilizing Lemma 2.1, from conditions (ii), (v)-(viii) and the assumption on $\left\{S_{n}\right\}$, we deduce that

$$
\lim _{n \rightarrow \infty}\left\|x_{n+1}-x_{n}\right\|=0
$$

Also, taking into account the boundedness of $\left\{G\left(x_{n}\right)\right\}$ and $\left\{S_{n} G\left(x_{n}\right)\right\}$, by Lemma 2.5 there exists a continuous strictly increasing function $g_{1}:[0, \infty) \rightarrow[0, \infty), g_{1}(0)=0$ such that for $p \in F$,

$$
\begin{aligned}
& \left\|z_{n}-p\right\|^{2} \\
& \quad=\left\|\sigma_{n}\left(G\left(x_{n}\right)-p\right)+\left(1-\sigma_{n}\right)\left(S_{n} G\left(x_{n}\right)-p\right)\right\|^{2} \\
& \quad \leq \sigma_{n}\left\|G\left(x_{n}\right)-p\right\|^{2}+\left(1-\sigma_{n}\right)\left\|S_{n} G\left(x_{n}\right)-p\right\|^{2}-\sigma_{n}\left(1-\sigma_{n}\right) g_{1}\left(\left\|G\left(x_{n}\right)-S_{n} G\left(x_{n}\right)\right\|\right) \\
& \quad \leq \sigma_{n}\left\|x_{n}-p\right\|^{2}+\left(1-\sigma_{n}\right)\left\|x_{n}-p\right\|^{2}-\sigma_{n}\left(1-\sigma_{n}\right) g_{1}\left(\left\|G\left(x_{n}\right)-S_{n} G\left(x_{n}\right)\right\|\right) \\
& \quad=\left\|x_{n}-p\right\|^{2}-\sigma_{n}\left(1-\sigma_{n}\right) g_{1}\left(\left\|G\left(x_{n}\right)-S_{n} G\left(x_{n}\right)\right\|\right),
\end{aligned}
$$

and hence

$$
\begin{aligned}
& \left\|y_{n}-p\right\|^{2} \\
& \quad=\left\|\alpha_{n}\left(f\left(x_{n}\right)-p\right)+\left(1-\alpha_{n}\right)\left(z_{n}-p\right)\right\|^{2} \\
& \quad \leq\left\|\left(1-\alpha_{n}\right)\left(z_{n}-p\right)\right\|^{2}+2 \alpha_{n}\left\langle f\left(x_{n}\right)-p, J\left(y_{n}-p\right)\right\rangle \\
& \quad \leq\left\|z_{n}-p\right\|^{2}+2 \alpha_{n}\left\|f\left(x_{n}\right)-p\right\|\left\|y_{n}-p\right\| \\
& \quad \leq\left\|x_{n}-p\right\|^{2}-\sigma_{n}\left(1-\sigma_{n}\right) g_{1}\left(\left\|G\left(x_{n}\right)-S_{n} G\left(x_{n}\right)\right\|\right)+2 \alpha_{n}\left\|f\left(x_{n}\right)-p\right\|\left\|y_{n}-p\right\| .
\end{aligned}
$$


Since $\left\{x_{n}\right\}$ and $\left\{S_{n} G\left(x_{n}\right)\right\}$ both are bounded, by Lemma 2.5 there exists a continuous strictly increasing function $g_{2}:[0, \infty) \rightarrow[0, \infty), g(0)=0$ such that

$$
\begin{aligned}
\left\|x_{n+1}-p\right\|^{2} & \left\|\left(\beta_{n}+\delta_{n}\right)\left(\frac{\beta_{n}}{\beta_{n}+\delta_{n}}\left(x_{n}-p\right)+\frac{\delta_{n}}{\beta_{n}+\delta_{n}}\left(S_{n+1} G\left(x_{n+1}\right)-p\right)\right)+\gamma_{n}\left(y_{n}-p\right)\right\|^{2} \\
\leq & \left(\beta_{n}+\delta_{n}\right)\left\|\frac{\beta_{n}}{\beta_{n}+\delta_{n}}\left(x_{n}-p\right)+\frac{\delta_{n}}{\beta_{n}+\delta_{n}}\left(S_{n+1} G\left(x_{n+1}\right)-p\right)\right\|^{2}+\gamma_{n}\left\|y_{n}-p\right\|^{2} \\
\leq & \left(\beta_{n}+\delta_{n}\right)\left[\frac{\beta_{n}}{\beta_{n}+\delta_{n}}\left\|x_{n}-p\right\|^{2}+\frac{\delta_{n}}{\beta_{n}+\delta_{n}}\left\|S_{n+1} G\left(x_{n+1}\right)-p\right\|\right. \\
& \left.-\frac{\beta_{n} \delta_{n}}{\left(\beta_{n}+\delta_{n}\right)^{2}} g_{2}\left(\left\|x_{n}-S_{n+1} G\left(x_{n+1}\right)\right\|\right)\right]+\gamma_{n}\left\|y_{n}-p\right\|^{2} \\
\leq & \beta_{n}\left\|x_{n}-p\right\|^{2}+\delta_{n}\left\|x_{n+1}-p\right\|^{2}-\frac{\beta_{n} \delta_{n}}{\beta_{n}+\delta_{n}} g_{2}\left(\left\|x_{n}-S_{n+1} G\left(x_{n+1}\right)\right\|\right)+\gamma_{n}\left\|y_{n}-p\right\|^{2} \\
\leq & \beta_{n}\left\|x_{n}-p\right\|^{2}+\delta_{n}\left\|x_{n+1}-p\right\|^{2}-\frac{\beta_{n} \delta_{n}}{\beta_{n}+\delta_{n}} g_{2}\left(\left\|x_{n}-S_{n+1} G\left(x_{n+1}\right)\right\|\right) \\
& +\gamma_{n}\left[\left\|x_{n}-p\right\|^{2}-\sigma_{n}\left(1-\sigma_{n}\right) g_{1}\left(\left\|G\left(x_{n}\right)-S_{n} G\left(x_{n}\right)\right\|\right)+2 \alpha_{n}\left\|f\left(x_{n}\right)-p\right\|\left\|y_{n}-p\right\|\right] \\
= & \left(\beta_{n}+\gamma_{n}\right)\left\|x_{n}-p\right\|^{2}+\delta_{n}\left\|x_{n+1}-p\right\|^{2}-\gamma_{n} \sigma_{n}\left(1-\sigma_{n}\right) g_{1}\left(\left\|G\left(x_{n}\right)-S_{n} G\left(x_{n}\right)\right\|\right) \\
& -\frac{\beta_{n} \delta_{n}}{\beta_{n}+\delta_{n}} g_{2}\left(\left\|x_{n}-S_{n+1} x_{n+1}\right\|\right)+2 \alpha_{n} \gamma_{n}\left\|f\left(x_{n}\right)-p\right\|\left\|y_{n}-p\right\|, \\
= &
\end{aligned}
$$

which immediately yields

$$
\begin{aligned}
& \gamma_{n} \sigma_{n}\left(1-\sigma_{n}\right) g_{1}\left(\left\|G\left(x_{n}\right)-S_{n} G\left(x_{n}\right)\right\|\right)+\beta_{n} \delta_{n} g_{2}\left(\left\|x_{n}-S_{n+1} G\left(x_{n+1}\right)\right\|\right) \\
& \quad \leq \gamma_{n} \sigma_{n}\left(1-\sigma_{n}\right) g_{1}\left(\left\|G\left(x_{n}\right)-S_{n} G\left(x_{n}\right)\right\|\right)+\frac{\beta_{n} \delta_{n}}{\beta_{n}+\delta_{n}} g\left(\left\|x_{n}-S_{n+1} x_{n+1}\right\|\right) \\
& \quad \leq\left(\beta_{n}+\gamma_{n}\right)\left(\left\|x_{n}-p\right\|^{2}-\left\|x_{n+1}-p\right\|^{2}\right)+2 \alpha_{n}\left\|f\left(x_{n}\right)-p\right\|\left\|y_{n}-p\right\| \\
& \leq\left(\left\|x_{n}-p\right\|+\left\|x_{n+1}-p\right\|\right)\left\|x_{n}-x_{n+1}\right\|+2 \alpha_{n}\left\|f\left(x_{n}\right)-p\right\|\left\|y_{n}-p\right\| .
\end{aligned}
$$

According to condition (iv), we have

$$
\liminf _{n \rightarrow \infty} \gamma_{n}=\liminf _{n \rightarrow \infty}\left(1-\beta_{n}-\delta_{n}\right)=1-\limsup _{n \rightarrow \infty}\left(\beta_{n}+\delta_{n}\right)>0
$$

Since $\alpha_{n} \rightarrow 0,\left\|x_{n+1}-x_{n}\right\| \rightarrow 0, \liminf _{n \rightarrow \infty} \beta_{n}>0$ and $\liminf _{n \rightarrow \infty} \delta_{n}>0$, we obtain from condition (i) that

$$
\lim _{n \rightarrow \infty} g_{1}\left(\left\|G\left(x_{n}\right)-S_{n} G\left(x_{n}\right)\right\|\right)=0 \quad \text { and } \quad \lim _{n \rightarrow \infty} g_{2}\left(\left\|x_{n}-S_{n+1} G\left(x_{n+1}\right)\right\|\right)=0
$$

Utilizing the properties of $g_{1}$ and $g_{2}$, we have

$$
\lim _{n \rightarrow \infty}\left\|G\left(x_{n}\right)-S_{n} G\left(x_{n}\right)\right\|=0 \text { and } \lim _{n \rightarrow \infty}\left\|x_{n}-S_{n+1} G\left(x_{n+1}\right)\right\|=0 .
$$


Thus, from (4.8) and (4.10) it follows that

$$
\begin{aligned}
\gamma_{n}\left\|y_{n}-x_{n}\right\| & =\left\|\left(x_{n+1}-x_{n}\right)-\delta_{n}\left(S_{n+1} G\left(x_{n+1}\right)-x_{n}\right)\right\| \\
& \leq\left\|x_{n+1}-x_{n}\right\|+\delta_{n}\left\|S_{n+1} G\left(x_{n+1}\right)-x_{n}\right\| \rightarrow 0 \text { as } n \rightarrow \infty .
\end{aligned}
$$

That is,

$$
\lim _{n \rightarrow \infty}\left\|y_{n}-x_{n}\right\|=0
$$

Since it follows from (4.1) that

$$
y_{n}-G\left(x_{n}\right)=\alpha_{n}\left(f\left(x_{n}\right)-G\left(x_{n}\right)\right)+\left(1-\alpha_{n}\right)\left(1-\sigma_{n}\right)\left(S_{n} G\left(x_{n}\right)-G\left(x_{n}\right)\right) \text {, }
$$

we conclude from (4.10) and $\alpha_{n} \rightarrow 0$ that

$$
\begin{aligned}
\left\|y_{n}-G\left(x_{n}\right)\right\| & =\left\|\alpha_{n}\left(f\left(x_{n}\right)-G\left(x_{n}\right)\right)+\left(1-\alpha_{n}\right)\left(1-\sigma_{n}\right)\left(S_{n} G\left(x_{n}\right)-G\left(x_{n}\right)\right)\right\| \\
& \leq \alpha_{n}\left\|f\left(x_{n}\right)-G\left(x_{n}\right)\right\|+\left(1-\alpha_{n}\right)\left(1-\sigma_{n}\right)\left\|S_{n} G\left(x_{n}\right)-G\left(x_{n}\right)\right\| \\
& \leq \alpha_{n}\left\|f\left(x_{n}\right)-G\left(x_{n}\right)\right\|+\left\|S_{n} G\left(x_{n}\right)-G\left(x_{n}\right)\right\| \\
& \rightarrow 0 \quad \text { as } n \rightarrow \infty .
\end{aligned}
$$

That is,

$$
\lim _{n \rightarrow \infty}\left\|y_{n}-G\left(x_{n}\right)\right\|=0 \text {. }
$$

This together with (4.11) leads to

$$
\lim _{n \rightarrow \infty}\left\|x_{n}-G\left(x_{n}\right)\right\|=0
$$

By (4.10) and Lemma 2.6, we have

$$
\begin{aligned}
\left\|S G\left(x_{n}\right)-G\left(x_{n}\right)\right\| & \leq\left\|S G\left(x_{n}\right)-S_{n} G\left(x_{n}\right)\right\|+\left\|S_{n} G\left(x_{n}\right)-G\left(x_{n}\right)\right\| \\
& \rightarrow 0 \quad \text { as } n \rightarrow \infty .
\end{aligned}
$$

In terms of (4.13) and (4.14), we have

$$
\begin{aligned}
\left\|x_{n}-S x_{n}\right\| & \leq\left\|x_{n}-G\left(x_{n}\right)\right\|+\left\|G\left(x_{n}\right)-S G\left(x_{n}\right)\right\|+\left\|S G\left(x_{n}\right)-S x_{n}\right\| \\
& \leq 2\left\|x_{n}-G\left(x_{n}\right)\right\|+\left\|G\left(x_{n}\right)-S G\left(x_{n}\right)\right\| \\
& \rightarrow 0 \quad \text { as } n \rightarrow \infty .
\end{aligned}
$$

Define a mapping $W x=(1-\theta) S x+\theta G(x)$, where $G$ is defined by $(1.7), \theta \in(0,1)$ is a constant. Then by Lemma 2.8 we have that $\operatorname{Fix}(W)=\operatorname{Fix}(S) \cap \operatorname{Fix}(G)=F$. We observe that

$$
\begin{aligned}
\left\|x_{n}-W x_{n}\right\| & =\left\|(1-\theta)\left(x_{n}-S x_{n}\right)+\theta\left(x_{n}-G\left(x_{n}\right)\right)\right\| \\
& \leq(1-\theta)\left\|x_{n}-S x_{n}\right\|+\theta\left\|x_{n}-G\left(x_{n}\right)\right\| .
\end{aligned}
$$


From (4.13) and (4.15), we obtain

$$
\lim _{n \rightarrow \infty}\left\|x_{n}-W x_{n}\right\|=0
$$

Utilizing the arguments similar to those of (3.17) in the proof of Theorem 3.1, we can obtain

$$
\limsup _{n \rightarrow \infty}\left\langle f(q)-q, J\left(x_{n}-q\right)\right\rangle \leq 0
$$

where $q=s-\lim _{t \rightarrow 0} x_{t}$ with $x_{t}$ being the fixed point of the contraction $x \mapsto t f(x)+(1-t) W x$; that is, $x_{t}$ solves the fixed point equation $x_{t}=t f\left(x_{t}\right)+(1-t) W x_{t}$. Noticing that $J$ is norm-toweak* uniformly continuous on bounded subsets of $X$, we deduce from (4.11) and (4.17) that

$$
\limsup _{n \rightarrow \infty}\left\langle f(q)-q, J\left(y_{n}-q\right)\right\rangle \leq 0
$$

Finally, let us show that $x_{n} \rightarrow q$ as $n \rightarrow \infty$. We observe that

$$
\begin{aligned}
\| y_{n}- & q \|^{2} \\
= & \alpha_{n}\left\langle f\left(x_{n}\right)-q, J\left(y_{n}-q\right)\right\rangle+\left(1-\alpha_{n}\right)\left\langle\sigma_{n}\left(G\left(x_{n}\right)-q\right)+\left(1-\sigma_{n}\right)\left(S_{n} G\left(x_{n}\right)-q\right), J\left(y_{n}-q\right)\right\rangle \\
= & \alpha_{n}\left\langle f\left(x_{n}\right)-f(q), J\left(y_{n}-q\right)\right\rangle+\alpha_{n}\left\langle f(q)-q, J\left(y_{n}-q\right)\right\rangle \\
& +\left(1-\alpha_{n}\right)\left\langle\sigma_{n}\left(G\left(x_{n}\right)-q\right)+\left(1-\sigma_{n}\right)\left(S_{n} G\left(x_{n}\right)-q\right), J\left(y_{n}-q\right)\right\rangle \\
\leq & \alpha_{n} \rho\left\|x_{n}-q\right\|\left\|y_{n}-q\right\|+\left(1-\alpha_{n}\right)\left\|\sigma_{n}\left(G\left(x_{n}\right)-q\right)+\left(1-\sigma_{n}\right)\left(S_{n} G\left(x_{n}\right)-q\right)\right\|\left\|y_{n}-q\right\| \\
& +\alpha_{n}\left\langle f(q)-q, J\left(y_{n}-q\right)\right\rangle \\
\leq & \alpha_{n} \rho\left\|x_{n}-q\right\|\left\|y_{n}-q\right\|+\left(1-\alpha_{n}\right)\left(\sigma_{n}\left\|G\left(x_{n}\right)-q\right\|+\left(1-\sigma_{n}\right)\left\|S_{n} G\left(x_{n}\right)-q\right\|\right)\left\|y_{n}-q\right\| \\
& +\alpha_{n}\left\langle f(q)-q, J\left(y_{n}-q\right)\right\rangle \\
\leq & \alpha_{n} \rho\left\|x_{n}-q\right\|\left\|y_{n}-q\right\|+\left(1-\alpha_{n}\right)\left(\sigma_{n}\left\|x_{n}-q\right\|+\left(1-\sigma_{n}\right)\left\|x_{n}-q\right\|\right)\left\|y_{n}-q\right\| \\
& +\alpha_{n}\left\langle f(q)-q, J\left(y_{n}-q\right)\right\rangle \\
= & \alpha_{n} \rho\left\|x_{n}-q\right\|\left\|y_{n}-q\right\|+\left(1-\alpha_{n}\right)\left\|x_{n}-q\right\|\left\|y_{n}-q\right\|+\alpha_{n}\left\langle f(q)-q, J\left(y_{n}-q\right)\right\rangle \\
= & \left(1-\alpha_{n}(1-\rho)\right)\left\|x_{n}-q\right\|\left\|y_{n}-q\right\|+\alpha_{n}\left\langle f(q)-q, J\left(y_{n}-q\right)\right\rangle \\
\leq & \frac{1-\alpha_{n}(1-\rho)}{2}\left(\left\|x_{n}-q\right\|^{2}+\left\|y_{n}-q\right\|^{2}\right)+\alpha_{n}\left\langle f(q)-q, J\left(y_{n}-q\right)\right\rangle \\
\leq & \frac{1-\alpha_{n}(1-\rho)}{2}\left\|x_{n}-q\right\|^{2}+\frac{1}{2}\left\|y_{n}-q\right\|^{2}+\alpha_{n}\left\langle f(q)-q, J\left(y_{n}-q\right)\right\rangle, \\
&
\end{aligned}
$$

which implies that

$$
\left\|y_{n}-q\right\|^{2} \leq\left(1-\alpha_{n}(1-\rho)\right)\left\|x_{n}-q\right\|^{2}+\alpha_{n}(1-\rho) \frac{2\left\langle f(q)-q, J\left(y_{n}-q\right)\right\rangle}{1-\rho} .
$$


Also, by (4.1) and the convexity of $\|\cdot\|^{2}$, we get

$$
\begin{aligned}
\left\|x_{n+1}-q\right\|^{2} & =\left\|\beta_{n}\left(x_{n}-q\right)+\gamma_{n}\left(y_{n}-q\right)+\delta_{n}\left(S_{n+1} G\left(x_{n+1}\right)-q\right)\right\|^{2} \\
& \leq \beta_{n}\left\|x_{n}-q\right\|^{2}+\gamma_{n}\left\|y_{n}-q\right\|^{2}+\delta_{n}\left\|x_{n+1}-q\right\|^{2},
\end{aligned}
$$

which together with (4.19) implies that

$$
\begin{aligned}
\left\|x_{n+1}-q\right\|^{2} \leq & \frac{\beta_{n}}{\beta_{n}+\gamma_{n}}\left\|x_{n}-q\right\|^{2}+\frac{\gamma_{n}}{\beta_{n}+\gamma_{n}}\left\|y_{n}-q\right\|^{2} \\
\leq & \frac{\beta_{n}}{\beta_{n}+\gamma_{n}}\left\|x_{n}-q\right\|^{2}+\frac{\gamma_{n}}{\beta_{n}+\gamma_{n}}\left[\left(1-\alpha_{n}(1-\rho)\right)\left\|x_{n}-q\right\|^{2}\right. \\
& \left.+\alpha_{n}(1-\rho) \frac{2\left\langle f(q)-q, J\left(y_{n}-q\right)\right\rangle}{1-\rho}\right] \\
= & \left(1-\frac{\alpha_{n} \gamma_{n}(1-\rho)}{\beta_{n}+\gamma_{n}}\right)\left\|x_{n}-q\right\|^{2} \\
& +\frac{\alpha_{n} \gamma_{n}(1-\rho)}{\beta_{n}+\gamma_{n}} \cdot \frac{2\left\langle f(q)-q, J\left(y_{n}-q\right)\right\rangle}{1-\rho} .
\end{aligned}
$$

Applying Lemma 2.1 to (4.20), we conclude that $x_{n} \rightarrow q$ as $n \rightarrow \infty$. This completes the proof.

Corollary 4.1 Let $C$ be a nonempty closed convex subset of a uniformly convex Banach space $X$ which has a uniformly Gâteaux differentiable norm. Let $\Pi_{C}$ be a sunny nonexpansive retraction from $X$ onto $C$. Let the mapping $B_{i}: C \rightarrow X$ be $\lambda_{i}$-strictly pseudocontractive and $\alpha_{i}$-strongly accretive with $\alpha_{i}+\lambda_{i} \geq 1$ for $i=1,2$. Let $f: C \rightarrow C$ be a contraction with coefficient $\rho \in(0,1)$. Let $S$ be a nonexpansive mapping of $C$ into itself such that $F=$ $\operatorname{Fix}(S) \cap \Omega \neq \emptyset$, where $\Omega$ is the fixed point set of the mapping $G=\Pi_{C}\left(I-\mu_{1} B_{1}\right) \Pi_{C}\left(I-\mu_{2} B_{2}\right)$. For arbitrarily given $x_{0} \in C$, let $\left\{x_{n}\right\}$ be the sequence generated by

$$
\left\{\begin{array}{l}
z_{n}=\sigma_{n} G\left(x_{n}\right)+\left(1-\sigma_{n}\right) S G\left(x_{n}\right), \\
y_{n}=\alpha_{n} f\left(x_{n}\right)+\left(1-\alpha_{n}\right) z_{n}, \\
x_{n+1}=\beta_{n} x_{n}+\gamma_{n} y_{n}+\delta_{n} S G\left(x_{n+1}\right), \quad \forall n \geq 0,
\end{array}\right.
$$

where $1-\frac{\lambda_{i}}{1+\lambda_{i}}\left(1-\sqrt{\frac{1-\alpha_{i}}{\lambda_{i}}}\right) \leq \mu_{i} \leq 1$ for $i=1,2$. Suppose that $\left\{\sigma_{n}\right\},\left\{\alpha_{n}\right\},\left\{\beta_{n}\right\},\left\{\gamma_{n}\right\}$ and $\left\{\delta_{n}\right\}$ are the sequences in $(0,1)$ satisfying the following conditions:

(i) $0<\liminf _{n \rightarrow \infty} \sigma_{n} \leq \limsup _{n \rightarrow \infty} \sigma_{n}<1$;

(ii) $\lim _{n \rightarrow \infty} \alpha_{n}=0$ and $\sum_{n=0}^{\infty} \alpha_{n}=\infty$;

(iii) $\beta_{n}+\gamma_{n}+\delta_{n}=1, \forall n \geq 0$, and $\liminf _{n \rightarrow \infty} \delta_{n}>0$;

(iv) $0<\liminf _{n \rightarrow \infty} \beta_{n} \leq \limsup _{n \rightarrow \infty}\left(\beta_{n}+\delta_{n}\right)<1$;

(v) $\sum_{n=1}^{\infty}\left|\frac{\gamma_{n}}{1-\beta_{n}}-\frac{\gamma_{n-1}}{1-\beta_{n-1}}\right|<\infty$ or $\lim _{n \rightarrow \infty} \frac{1}{\alpha_{n}}\left|\frac{\gamma_{n}}{1-\beta_{n}}-\frac{\gamma_{n-1}}{1-\beta_{n-1}}\right|=0$;

(vi) $\sum_{n=1}^{\infty}\left|\alpha_{n}-\alpha_{n-1}\right|<\infty$ or $\lim _{n \rightarrow \infty} \alpha_{n-1} / \alpha_{n}=1$;

(vii) $\sum_{n=1}^{\infty}\left|\beta_{n}-\beta_{n-1}\right|<\infty$ or $\lim _{n \rightarrow \infty}\left|\beta_{n}-\beta_{n-1}\right| / \alpha_{n}=0$;

(viii) $\sum_{n=1}^{\infty}\left|\sigma_{n}-\sigma_{n-1}\right|<\infty$ or $\lim _{n \rightarrow \infty}\left|\sigma_{n}-\sigma_{n-1}\right| / \alpha_{n}=0$.

Then $\left\{x_{n}\right\}$ converges strongly to $q \in F$, which solves the following VIP:

$$
\langle q-f(q), J(q-p)\rangle \leq 0, \quad \forall p \in F
$$




\section{Two-step explicit iterative algorithm}

In this section, we introduce our two-step explicit iterative algorithm and show strong convergence of the purposed algorithm.

Theorem 5.1 Let $C$ be a nonempty closed convex subset of a uniformly convex Banach space $X$ which has a uniformly Gâteaux differentiable norm. Let $\Pi_{C}$ be a sunny nonexpansive retraction from $X$ onto $C$. Let the mapping $B_{i}: C \rightarrow X$ be $\lambda_{i}$-strictly pseudocontractive and $\alpha_{i}$-strongly accretive with $\alpha_{i}+\lambda_{i} \geq 1$ for $i=1,2$. Let $f: C \rightarrow C$ be a contraction with coefficient $\rho \in(0,1)$. Let $\left\{S_{n}\right\}_{n=0}^{\infty}$ be an infinite family of nonexpansive mappings of $C$ into itself such that $F=\bigcap_{i=0}^{\infty} \operatorname{Fix}\left(S_{i}\right) \cap \Omega \neq \emptyset$, where $\Omega$ is the fixed point set of the mapping $G=\Pi_{C}\left(I-\mu_{1} B_{1}\right) \Pi_{C}\left(I-\mu_{2} B_{2}\right)$. For arbitrarily given $x_{0} \in C$, let $\left\{x_{n}\right\}$ be the sequence generated by

$$
\left\{\begin{array}{l}
y_{n}=\alpha_{n} f\left(x_{n}\right)+\left(1-\alpha_{n}\right) G\left(x_{n}\right), \\
x_{n+1}=\beta_{n} x_{n}+\gamma_{n} y_{n}+\delta_{n} S_{n} y_{n}, \quad \forall n \geq 0,
\end{array}\right.
$$

where $1-\frac{\lambda_{i}}{1+\lambda_{i}}\left(1-\sqrt{\frac{1-\alpha_{i}}{\lambda_{i}}}\right) \leq \mu_{i} \leq 1$ for $i=1,2$. Suppose that $\left\{\alpha_{n}\right\},\left\{\beta_{n}\right\},\left\{\gamma_{n}\right\}$ and $\left\{\delta_{n}\right\}$ are the sequences in $(0,1)$ satisfying the following conditions:

(i) $\lim _{n \rightarrow \infty} \alpha_{n}=0$ and $\sum_{n=0}^{\infty} \alpha_{n}=\infty$;

(ii) $\beta_{n}+\gamma_{n}+\delta_{n}=1, \forall n \geq 0$, and $\liminf _{n \rightarrow \infty} \delta_{n}>0$;

(iii) $0<\liminf _{n \rightarrow \infty} \beta_{n} \leq \limsup _{n \rightarrow \infty}\left(\beta_{n}+\delta_{n}\right)<1$;

(iv) $\lim _{n \rightarrow \infty}\left|\frac{\gamma_{n}}{1-\beta_{n}}-\frac{\gamma_{n-1}}{1-\beta_{n-1}}\right|=0$.

Assume that $\sum_{n=1}^{\infty} \sup _{x \in D}\left\|S_{n} x-S_{n-1} x\right\|<\infty$ for any bounded subset $D$ of $C$ and let $S$ be a mapping of $C$ into itself defined by $S x=\lim _{n \rightarrow \infty} S_{n} x$ for all $x \in C$ and suppose that $\operatorname{Fix}(S)=\bigcap_{i=0}^{\infty} \operatorname{Fix}\left(S_{i}\right)$. Then $\left\{x_{n}\right\}$ converges strongly to $q \in F$, which solves the following VIP:

$$
\langle q-f(q), J(q-p)\rangle \leq 0, \quad \forall p \in F
$$

Proof Take a fixed $p \in F$ arbitrarily. Then by Lemma 2.11 we know that $p=G(p)$. Moreover, by Lemma 2.10 we have

$$
\begin{aligned}
\left\|y_{n}-p\right\| & =\left\|\alpha_{n}\left(f\left(x_{n}\right)-p\right)+\left(1-\alpha_{n}\right)\left(G\left(x_{n}\right)-p\right)\right\| \\
& \leq \alpha_{n}\left\|f\left(x_{n}\right)-f(p)\right\|+\alpha_{n}\|f(p)-p\|+\left(1-\alpha_{n}\right)\left\|G\left(x_{n}\right)-p\right\| \\
& \leq \alpha_{n} \rho\left\|x_{n}-p\right\|+\alpha_{n}\|f(p)-p\|+\left(1-\alpha_{n}\right)\left\|x_{n}-p\right\| \\
& =\left(1-\alpha_{n}(1-\rho)\right)\left\|x_{n}-p\right\|+\alpha_{n}\|f(p)-p\| .
\end{aligned}
$$

From (5.3) we obtain

$$
\begin{aligned}
\left\|x_{n+1}-p\right\| & =\left\|\beta_{n}\left(x_{n}-p\right)+\gamma_{n}\left(y_{n}-p\right)+\delta_{n}\left(S_{n} y_{n}-p\right)\right\| \\
& \leq \beta_{n}\left\|x_{n}-p\right\|+\gamma_{n}\left\|y_{n}-p\right\|+\delta_{n}\left\|y_{n}-p\right\| \\
& =\beta_{n}\left\|x_{n}-p\right\|+\left(1-\beta_{n}\right)\left\|y_{n}-p\right\| \\
& \leq \beta_{n}\left\|x_{n}-p\right\|+\left(1-\beta_{n}\right)\left[\left(1-\alpha_{n}(1-\rho)\right)\left\|x_{n}-p\right\|+\alpha_{n}\|f(p)-p\|\right]
\end{aligned}
$$




$$
\begin{aligned}
& =\left[1-\left(1-\beta_{n}\right)(1-\rho) \alpha_{n}\right]\left\|x_{n}-p\right\|+\left(1-\beta_{n}\right)(1-\rho) \alpha_{n} \cdot \frac{\|f(p)-p\|}{1-\rho} \\
& \leq \max \left\{\left\|x_{0}-p\right\|, \frac{\|f(p)-p\|}{1-\rho}\right\},
\end{aligned}
$$

which implies that $\left\{x_{n}\right\}$ is bounded. By Lemma 2.10 we know from (5.3) that $\left\{G\left(x_{n}\right)\right\}$ and $\left\{y_{n}\right\}$ both are bounded.

Let us show that $\left\|x_{n+1}-x_{n}\right\| \rightarrow 0$ and $\left\|x_{n}-y_{n}\right\| \rightarrow 0$ as $n \rightarrow \infty$. As a matter of fact, from (5.1) we have

$$
y_{n}-y_{n-1}=\alpha_{n}\left(f\left(x_{n}\right)-f\left(x_{n-1}\right)\right)+\left(\alpha_{n}-\alpha_{n-1}\right)\left(f\left(x_{n-1}\right)-G\left(x_{n-1}\right)\right)+\left(1-\alpha_{n}\right)\left(G\left(x_{n}\right)-G\left(x_{n-1}\right)\right) .
$$

It follows that

$$
\begin{aligned}
\left\|y_{n}-y_{n-1}\right\| \leq & \alpha_{n}\left\|f\left(x_{n}\right)-f\left(x_{n-1}\right)\right\|+\left|\alpha_{n}-\alpha_{n-1}\right|\left\|f\left(x_{n-1}\right)-G\left(x_{n-1}\right)\right\| \\
& +\left(1-\alpha_{n}\right)\left\|G\left(x_{n}\right)-G\left(x_{n-1}\right)\right\| \\
\leq & \alpha_{n} \rho\left\|x_{n}-x_{n-1}\right\|+\left|\alpha_{n}-\alpha_{n-1}\right|\left\|f\left(x_{n-1}\right)-G\left(x_{n-1}\right)\right\| \\
& +\left(1-\alpha_{n}\right)\left\|x_{n}-x_{n-1}\right\| \\
= & \left(1-(1-\rho) \alpha_{n}\right)\left\|x_{n}-x_{n-1}\right\|+\left|\alpha_{n}-\alpha_{n-1}\right|\left\|f\left(x_{n-1}\right)-G\left(x_{n-1}\right)\right\| .
\end{aligned}
$$

Now, we write $x_{n}=\beta_{n-1} x_{n-1}+\left(1-\beta_{n-1}\right) v_{n-1}, \forall n \geq 1$, where $v_{n-1}=\frac{x_{n}-\beta_{n-1} x_{n-1}}{1-\beta_{n-1}}$. It follows that for all $n \geq 1$,

$$
\begin{aligned}
v_{n}-v_{n-1}= & \frac{x_{n+1}-\beta_{n} x_{n}}{1-\beta_{n}}-\frac{x_{n}-\beta_{n-1} x_{n-1}}{1-\beta_{n-1}} \\
= & \frac{\gamma_{n} y_{n}+\delta_{n} S_{n} y_{n}}{1-\beta_{n}}-\frac{\gamma_{n-1} y_{n-1}+\delta_{n-1} S_{n-1} y_{n-1}}{1-\beta_{n-1}} \\
= & \frac{\gamma_{n}\left(y_{n}-y_{n-1}\right)+\delta_{n}\left(S_{n} y_{n}-S_{n-1} y_{n-1}\right)}{1-\beta_{n}} \\
& +\left(\frac{\gamma_{n}}{1-\beta_{n}}-\frac{\gamma_{n-1}}{1-\beta_{n-1}}\right) y_{n-1}+\left(\frac{\delta_{n}}{1-\beta_{n}}-\frac{\delta_{n-1}}{1-\beta_{n-1}}\right) S_{n-1} y_{n-1} .
\end{aligned}
$$

This together with (5.4) implies that

$$
\begin{aligned}
\| v_{n} & -v_{n-1} \| \\
\leq & \frac{\left\|\gamma_{n}\left(y_{n}-y_{n-1}\right)+\delta_{n}\left(S_{n} y_{n}-S_{n-1} y_{n-1}\right)\right\|}{1-\beta_{n}}+\left|\frac{\gamma_{n}}{1-\beta_{n}}-\frac{\gamma_{n-1}}{1-\beta_{n-1}}\right|\left\|y_{n-1}\right\| \\
& +\left|\frac{\delta_{n}}{1-\beta_{n}}-\frac{\delta_{n-1}}{1-\beta_{n-1}}\right|\left\|S_{n-1} y_{n-1}\right\| \\
\leq & \frac{\gamma_{n}\left\|y_{n}-y_{n-1}\right\|+\delta_{n}\left(\left\|S_{n} y_{n}-S_{n} y_{n-1}\right\|+\left\|S_{n} y_{n-1}-S_{n-1} y_{n-1}\right\|\right)}{1-\beta_{n}} \\
& +\left|\frac{\gamma_{n}}{1-\beta_{n}}-\frac{\gamma_{n-1}}{1-\beta_{n-1}}\right|\left\|y_{n-1}\right\|+\left|\frac{\gamma_{n}}{1-\beta_{n}}-\frac{\gamma_{n-1}}{1-\beta_{n-1}}\right|\left\|S_{n-1} y_{n-1}\right\| \\
\leq & \frac{\gamma_{n}\left\|y_{n}-y_{n-1}\right\|+\delta_{n}\left\|y_{n}-y_{n-1}\right\|}{\gamma_{n}+\delta_{n}}+\frac{\delta_{n}}{\gamma_{n}+\delta_{n}}\left\|S_{n} y_{n-1}-S_{n-1} y_{n-1}\right\|
\end{aligned}
$$




$$
\begin{aligned}
& +\left|\frac{\gamma_{n}}{1-\beta_{n}}-\frac{\gamma_{n-1}}{1-\beta_{n-1}}\right|\left(\left\|y_{n-1}\right\|+\left\|S_{n-1} y_{n-1}\right\|\right) \\
\leq & \left\|y_{n}-y_{n-1}\right\|+\left\|S_{n} y_{n-1}-S_{n-1} y_{n-1}\right\|+\left|\frac{\gamma_{n}}{1-\beta_{n}}-\frac{\gamma_{n-1}}{1-\beta_{n-1}}\right|\left(\left\|y_{n-1}\right\|+\left\|S_{n-1} y_{n-1}\right\|\right) \\
\leq & \left(1-(1-\rho) \alpha_{n}\right)\left\|x_{n}-x_{n-1}\right\|+\left|\alpha_{n}-\alpha_{n-1}\right|\left\|f\left(x_{n-1}\right)-G\left(x_{n-1}\right)\right\| \\
& +\left\|S_{n} y_{n-1}-S_{n-1} y_{n-1}\right\|+\left|\frac{\gamma_{n}}{1-\beta_{n}}-\frac{\gamma_{n-1}}{1-\beta_{n-1}}\right|\left(\left\|y_{n-1}\right\|+\left\|S_{n-1} y_{n-1}\right\|\right) \\
\leq & \left(1-(1-\rho) \alpha_{n}\right)\left\|x_{n}-x_{n-1}\right\|+\left|\alpha_{n}-\alpha_{n-1}\right|\left(\left\|f\left(x_{n-1}\right)\right\|+\left\|G\left(x_{n-1}\right)\right\|\right) \\
& +\left\|S_{n} y_{n-1}-S_{n-1} y_{n-1}\right\|+\left|\frac{\gamma_{n}}{1-\beta_{n}}-\frac{\gamma_{n-1}}{1-\beta_{n-1}}\right|\left(\left\|y_{n-1}\right\|+\left\|S_{n-1} y_{n-1}\right\|\right) \\
\leq & \left\|x_{n}-x_{n-1}\right\|+M\left(\left|\alpha_{n}-\alpha_{n-1}\right|+\left|\frac{\gamma_{n}}{1-\beta_{n}}-\frac{\gamma_{n-1}}{1-\beta_{n-1}}\right|\right)+\left\|S_{n} y_{n-1}-S_{n-1} y_{n-1}\right\|,
\end{aligned}
$$

where $\sup _{n \geq 0}\left\{\left\|f\left(x_{n}\right)\right\|+\left\|G\left(x_{n}\right)\right\|+\left\|y_{n}\right\|+\left\|S_{n} y_{n}\right\|\right\} \leq M$ for some $M>0$. Since $\alpha_{n} \rightarrow 0$, $\left|\frac{\gamma_{n}}{1-\beta_{n}}-\frac{\gamma_{n-1}}{1-\beta_{n-1}}\right| \rightarrow 0$ and the assumption on $\left\{S_{n}\right\}$, we have

$$
\limsup _{n \rightarrow \infty}\left(\left\|v_{n}-v_{n-1}\right\|-\left\|x_{n}-x_{n-1}\right\|\right) \leq 0 .
$$

Utilizing Lemma 2.3, from condition (iii) we obtain that

$$
\lim _{n \rightarrow \infty}\left\|v_{n}-x_{n}\right\|=0
$$

So, we get

$$
\lim _{n \rightarrow \infty}\left\|x_{n+1}-x_{n}\right\|=\lim _{n \rightarrow \infty}\left(1-\beta_{n}\right)\left\|v_{n}-x_{n}\right\|=0
$$

Also, we note that for $p \in F$,

$$
\begin{aligned}
\left\|y_{n}-p\right\|^{2} & =\left\|\alpha_{n}\left(f\left(x_{n}\right)-p\right)+\left(1-\alpha_{n}\right)\left(G\left(x_{n}\right)-G(p)\right)\right\|^{2} \\
& \leq\left\|\left(1-\alpha_{n}\right)\left(G\left(x_{n}\right)-G(p)\right)\right\|^{2}+2 \alpha_{n}\left\langle f\left(x_{n}\right)-p, J\left(y_{n}-p\right)\right\rangle \\
& \leq\left\|x_{n}-p\right\|^{2}+2 \alpha_{n}\left\|f\left(x_{n}\right)-p\right\|\left\|y_{n}-p\right\| .
\end{aligned}
$$

Since $\left\{x_{n}\right\}$ and $\left\{y_{n}\right\}$ both are bounded, by Lemma 2.5 there exists a continuous strictly increasing function $g:[0, \infty) \rightarrow[0, \infty), g(0)=0$ such that

$$
\begin{aligned}
& \left\|x_{n+1}-p\right\|^{2} \\
& =\left\|\left(\beta_{n}+\delta_{n}\right)\left(\frac{\beta_{n}}{\beta_{n}+\delta_{n}}\left(x_{n}-p\right)+\frac{\delta_{n}}{\beta_{n}+\delta_{n}}\left(S_{n} y_{n}-p\right)\right)+\gamma_{n}\left(y_{n}-p\right)\right\|^{2} \\
& \leq\left(\beta_{n}+\delta_{n}\right)\left\|\frac{\beta_{n}}{\beta_{n}+\delta_{n}}\left(x_{n}-p\right)+\frac{\delta_{n}}{\beta_{n}+\delta_{n}}\left(S_{n} y_{n}-p\right)\right\|^{2}+\gamma_{n}\left\|y_{n}-p\right\|^{2} \\
& \leq \\
& \quad\left(\beta_{n}+\delta_{n}\right)\left[\frac{\beta_{n}}{\beta_{n}+\delta_{n}}\left\|x_{n}-p\right\|^{2}+\frac{\delta_{n}}{\beta_{n}+\delta_{n}}\left\|S_{n} y_{n}-p\right\|-\frac{\beta_{n} \delta_{n}}{\left(\beta_{n}+\delta_{n}\right)^{2}} g\left(\left\|x_{n}-S_{n} y_{n}\right\|\right)\right] \\
& \quad+\gamma_{n}\left\|y_{n}-p\right\|^{2}
\end{aligned}
$$




$$
\begin{aligned}
\leq & \beta_{n}\left\|x_{n}-p\right\|^{2}+\delta_{n}\left\|S_{n} y_{n}-p\right\|^{2}-\frac{\beta_{n} \delta_{n}}{\beta_{n}+\delta_{n}} g\left(\left\|x_{n}-S_{n} y_{n}\right\|\right)+\gamma_{n}\left\|y_{n}-p\right\|^{2} \\
\leq & \beta_{n}\left\|x_{n}-p\right\|^{2}+\delta_{n}\left\|y_{n}-p\right\|^{2}-\frac{\beta_{n} \delta_{n}}{\beta_{n}+\delta_{n}} g\left(\left\|x_{n}-S_{n} y_{n}\right\|\right)+\gamma_{n}\left\|y_{n}-p\right\|^{2} \\
= & \beta_{n}\left\|x_{n}-p\right\|^{2}+\left(1-\beta_{n}\right)\left\|y_{n}-p\right\|^{2}-\frac{\beta_{n} \delta_{n}}{\beta_{n}+\delta_{n}} g\left(\left\|x_{n}-S_{n} y_{n}\right\|\right) \\
\leq & \beta_{n}\left\|x_{n}-p\right\|^{2}+\left(1-\beta_{n}\right)\left[\left\|x_{n}-p\right\|^{2}+2 \alpha_{n}\left\|f\left(x_{n}\right)-p\right\|\left\|y_{n}-p\right\|\right] \\
& -\frac{\beta_{n} \delta_{n}}{\beta_{n}+\delta_{n}} g\left(\left\|x_{n}-S_{n} y_{n}\right\|\right) \\
\leq & \left\|x_{n}-p\right\|^{2}+2 \alpha_{n}\left\|f\left(x_{n}\right)-p\right\|\left\|y_{n}-p\right\|-\frac{\beta_{n} \delta_{n}}{\beta_{n}+\delta_{n}} g\left(\left\|x_{n}-S_{n} y_{n}\right\|\right)
\end{aligned}
$$

which immediately yields

$$
\begin{aligned}
\beta_{n} \delta_{n} g\left(\left\|x_{n}-S_{n} y_{n}\right\|\right) & \leq \frac{\beta_{n} \delta_{n}}{\beta_{n}+\delta_{n}} g\left(\left\|x_{n}-S_{n} y_{n}\right\|\right) \\
& \leq\left\|x_{n}-p\right\|^{2}-\left\|x_{n+1}-p\right\|^{2}+2 \alpha_{n}\left\|f\left(x_{n}\right)-p\right\|\left\|y_{n}-p\right\| \\
& \leq\left(\left\|x_{n}-p\right\|+\left\|x_{n+1}-p\right\|\right)\left\|x_{n}-x_{n+1}\right\|+2 \alpha_{n}\left\|f\left(x_{n}\right)-p\right\|\left\|y_{n}-p\right\| .
\end{aligned}
$$

Since $\alpha_{n} \rightarrow 0,\left\|x_{n+1}-x_{n}\right\| \rightarrow 0, \liminf _{n \rightarrow \infty} \beta_{n}>0$ and $\liminf _{n \rightarrow \infty} \delta_{n}>0$, we get $\lim _{n \rightarrow \infty} g\left(\left\|x_{n}-S_{n} y_{n}\right\|\right)=0$ and hence

$$
\lim _{n \rightarrow \infty}\left\|x_{n}-S_{n} y_{n}\right\|=0
$$

In the meantime, according to condition (iii), we have

$$
\liminf _{n \rightarrow \infty} \gamma_{n}=\liminf _{n \rightarrow \infty}\left(1-\beta_{n}-\delta_{n}\right)=1-\limsup _{n \rightarrow \infty}\left(\beta_{n}+\delta_{n}\right)>0 .
$$

Thus, from (5.7) and (5.9) it follows that

$$
\begin{aligned}
\gamma_{n}\left\|y_{n}-x_{n}\right\| & =\left\|\left(x_{n+1}-x_{n}\right)-\delta_{n}\left(S_{n} y_{n}-x_{n}\right)\right\| \\
& \leq\left\|x_{n+1}-x_{n}\right\|+\delta_{n}\left\|S_{n} y_{n}-x_{n}\right\| \rightarrow 0 \quad \text { as } n \rightarrow \infty .
\end{aligned}
$$

That is,

$$
\lim _{n \rightarrow \infty}\left\|y_{n}-x_{n}\right\|=0
$$

This together with (5.1) leads to

$$
\begin{aligned}
\left(1-\alpha_{n}\right)\left\|G\left(x_{n}\right)-x_{n}\right\| & =\left\|\left(y_{n}-x_{n}\right)-\alpha_{n}\left(f\left(x_{n}\right)-x_{n}\right)\right\| \\
& \leq\left\|y_{n}-x_{n}\right\|+\alpha_{n}\left\|f\left(x_{n}\right)-x_{n}\right\| \rightarrow 0 \quad \text { as } n \rightarrow \infty .
\end{aligned}
$$

That is,

$$
\lim _{n \rightarrow \infty}\left\|x_{n}-G\left(x_{n}\right)\right\|=0
$$


On the other hand, we observe that

$$
y_{n}-G\left(x_{n}\right)=\alpha_{n}\left(f\left(x_{n}\right)-G\left(x_{n}\right)\right),
$$

which together with $\alpha_{n} \rightarrow 0$ implies that

$$
\lim _{n \rightarrow \infty}\left\|y_{n}-G\left(x_{n}\right)\right\|=0
$$

We note that

$$
\begin{aligned}
\left\|S_{n} G\left(x_{n}\right)-G\left(x_{n}\right)\right\| & \leq\left\|S_{n} G\left(x_{n}\right)-S_{n} y_{n}\right\|+\left\|S_{n} y_{n}-x_{n}\right\|+\left\|x_{n}-G\left(x_{n}\right)\right\| \\
& \leq\left\|G\left(x_{n}\right)-y_{n}\right\|+\left\|S_{n} y_{n}-x_{n}\right\|+\left\|x_{n}-G\left(x_{n}\right)\right\| .
\end{aligned}
$$

From (5.9), (5.11) and (5.12), we obtain

$$
\lim _{n \rightarrow \infty}\left\|S_{n} G\left(x_{n}\right)-G\left(x_{n}\right)\right\|=0 .
$$

By (5.13) and Lemma 2.6, we have

$$
\begin{aligned}
\left\|S G\left(x_{n}\right)-G\left(x_{n}\right)\right\| & \leq\left\|S G\left(x_{n}\right)-S_{n} G\left(x_{n}\right)\right\|+\left\|S_{n} G\left(x_{n}\right)-G\left(x_{n}\right)\right\| \\
& \rightarrow 0 \quad \text { as } n \rightarrow \infty
\end{aligned}
$$

In terms of (5.11) and (5.14), we have

$$
\begin{aligned}
\left\|x_{n}-S x_{n}\right\| & \leq\left\|x_{n}-G\left(x_{n}\right)\right\|+\left\|G\left(x_{n}\right)-S G\left(x_{n}\right)\right\|+\left\|S G\left(x_{n}\right)-S x_{n}\right\| \\
& \leq 2\left\|x_{n}-G\left(x_{n}\right)\right\|+\left\|G\left(x_{n}\right)-S G\left(x_{n}\right)\right\| \\
& \rightarrow 0 \quad \text { as } n \rightarrow \infty .
\end{aligned}
$$

Define a mapping $W x=(1-\theta) S x+\theta G(x)$, where $G$ is defined by $(1.7), \theta \in(0,1)$ is a constant. Then by Lemma 2.8 we have that $\operatorname{Fix}(W)=\operatorname{Fix}(S) \cap \operatorname{Fix}(G)=F$. We observe that

$$
\begin{aligned}
\left\|x_{n}-W x_{n}\right\| & =\left\|(1-\theta)\left(x_{n}-S x_{n}\right)+\theta\left(x_{n}-G\left(x_{n}\right)\right)\right\| \\
& \leq(1-\theta)\left\|x_{n}-S x_{n}\right\|+\theta\left\|x_{n}-G\left(x_{n}\right)\right\| .
\end{aligned}
$$

From (5.11) and (5.15), we obtain

$$
\lim _{n \rightarrow \infty}\left\|x_{n}-W x_{n}\right\|=0
$$

Utilizing the arguments similar to those of (3.17) in the proof of Theorem 3.1, we can obtain

$$
\limsup _{n \rightarrow \infty}\left\langle f(q)-q, J\left(x_{n}-q\right)\right\rangle \leq 0,
$$


where $q=s-\lim _{t \rightarrow 0} x_{t}$ with $x_{t}$ being the fixed point of the contraction $x \mapsto t f(x)+(1-t) W x$; that is, $x_{t}$ solves the fixed point equation $x_{t}=t f\left(x_{t}\right)+(1-t) W x_{t}$. Noticing that $J$ is norm-toweak* uniformly continuous on bounded subsets of $X$, we deduce from (5.10) and (5.17) that

$$
\limsup _{n \rightarrow \infty}\left\langle f(q)-q, J\left(y_{n}-q\right)\right\rangle \leq 0
$$

Finally, let us show that $x_{n} \rightarrow q$ as $n \rightarrow \infty$. We observe that

$$
\begin{aligned}
\| y_{n} & -q \|^{2} \\
& =\alpha_{n}\left\langle f\left(x_{n}\right)-q, J\left(y_{n}-q\right)\right\rangle+\left(1-\alpha_{n}\right)\left\langle G\left(x_{n}\right)-q, J\left(y_{n}-q\right)\right\rangle \\
& =\alpha_{n}\left\langle f\left(x_{n}\right)-f(q), J\left(y_{n}-q\right)\right\rangle+\alpha_{n}\left\langle f(q)-q, J\left(y_{n}-q\right)\right\rangle+\left(1-\alpha_{n}\right)\left\langle G\left(x_{n}\right)-q, J\left(y_{n}-q\right)\right\rangle \\
& \leq \alpha_{n} \rho\left\|x_{n}-q\right\|\left\|y_{n}-q\right\|+\left(1-\alpha_{n}\right)\left\|G\left(x_{n}\right)-q\right\|\left\|y_{n}-q\right\|+\alpha_{n}\left\langle f(q)-q, J\left(y_{n}-q\right)\right\rangle \\
& \leq \alpha_{n} \rho\left\|x_{n}-q\right\|\left\|y_{n}-q\right\|+\left(1-\alpha_{n}\right)\left\|x_{n}-q\right\|\left\|y_{n}-q\right\|+\alpha_{n}\left\langle f(q)-q, J\left(y_{n}-q\right)\right\rangle \\
& =\left[1-\alpha_{n}(1-\rho)\right]\left\|x_{n}-q\right\|\left\|y_{n}-q\right\|+\alpha_{n}\left\langle f(q)-q, J\left(y_{n}-q\right)\right\rangle \\
& \leq \frac{1-\alpha_{n}(1-\rho)}{2}\left(\left\|x_{n}-q\right\|^{2}+\left\|y_{n}-q\right\|^{2}\right)+\alpha_{n}\left\langle f(q)-q, J\left(y_{n}-q\right)\right\rangle \\
& \leq \frac{1-\alpha_{n}(1-\rho)}{2}\left\|x_{n}-q\right\|^{2}+\frac{1}{2}\left\|y_{n}-q\right\|^{2}+\alpha_{n}\left\langle f(q)-q, J\left(y_{n}-q\right)\right\rangle,
\end{aligned}
$$

which implies that

$$
\left\|y_{n}-q\right\|^{2} \leq\left[1-\alpha_{n}(1-\rho)\right]\left\|x_{n}-q\right\|^{2}+\alpha_{n}(1-\rho) \frac{2\left\langle f(q)-q, J\left(y_{n}-q\right)\right\rangle}{1-\rho} .
$$

Also, by the convexity of $\|\cdot\|^{2}$ and (5.1), we get

$$
\begin{aligned}
\left\|x_{n+1}-q\right\|^{2} & \leq \beta_{n}\left\|x_{n}-q\right\|^{2}+\gamma_{n}\left\|y_{n}-q\right\|^{2}+\delta_{n}\left\|y_{n}-q\right\|^{2} \\
& =\beta_{n}\left\|x_{n}-q\right\|^{2}+\left(1-\beta_{n}\right)\left\|y_{n}-q\right\|^{2} .
\end{aligned}
$$

It follows from (5.19) that

$$
\begin{aligned}
\left\|x_{n+1}-q\right\|^{2} \leq & {\left[1-\alpha_{n}\left(1-\beta_{n}\right)(1-\rho)\right]\left\|x_{n}-q\right\|^{2} } \\
& +\alpha_{n}\left(1-\beta_{n}\right)(1-\rho) \frac{2\left\langle f(q)-q, J\left(y_{n}-q\right)\right\rangle}{1-\rho} .
\end{aligned}
$$

Applying Lemma 2.1 to (5.20), we obtain that $x_{n} \rightarrow q$ as $n \rightarrow \infty$. This completes the proof.

Corollary 5.1 Let $C$ be a nonempty closed convex subset of a uniformly convex Banach space $X$ which has a uniformly Gâteaux differentiable norm. Let $\Pi_{C}$ be a sunny nonexpansive retraction from $X$ onto $C$. Let the mapping $B_{i}: C \rightarrow X$ be $\lambda_{i}$-strictly pseudocontractive and $\alpha_{i}$-strongly accretive with $\alpha_{i}+\lambda_{i} \geq 1$ for $i=1,2$. Let $f: C \rightarrow C$ be a contraction with coefficient $\rho \in(0,1)$. Let $S$ be a nonexpansive mapping of $C$ into itself such that $F=$ 
$\operatorname{Fix}(S) \cap \Omega \neq \emptyset$, where $\Omega$ is the fixed point set of the mapping $G=\Pi_{C}\left(I-\mu_{1} B_{1}\right) \Pi_{C}\left(I-\mu_{2} B_{2}\right)$. For arbitrarily given $x_{0} \in C$, let $\left\{x_{n}\right\}$ be the sequence generated by

$$
\left\{\begin{array}{l}
y_{n}=\alpha_{n} f\left(x_{n}\right)+\left(1-\alpha_{n}\right) G\left(x_{n}\right), \\
x_{n+1}=\beta_{n} x_{n}+\gamma_{n} y_{n}+\delta_{n} S y_{n}, \quad \forall n \geq 0,
\end{array}\right.
$$

where $1-\frac{\lambda_{i}}{1+\lambda_{i}}\left(1-\sqrt{\frac{1-\alpha_{i}}{\lambda_{i}}}\right) \leq \mu_{i} \leq 1$ for $i=1,2$. Suppose that $\left\{\alpha_{n}\right\},\left\{\beta_{n}\right\},\left\{\gamma_{n}\right\}$ and $\left\{\delta_{n}\right\}$ are the sequences in $(0,1)$ satisfying the following conditions:

(i) $\lim _{n \rightarrow \infty} \alpha_{n}=0$ and $\sum_{n=0}^{\infty} \alpha_{n}=\infty$;

(ii) $\beta_{n}+\gamma_{n}+\delta_{n}=1, \forall n \geq 0$, and $\liminf _{n \rightarrow \infty} \delta_{n}>0$;

(iii) $0<\liminf _{n \rightarrow \infty} \beta_{n} \leq \lim \sup _{n \rightarrow \infty}\left(\beta_{n}+\delta_{n}\right)<1$;

(iv) $\lim _{n \rightarrow \infty}\left|\frac{\gamma_{n}}{1-\beta_{n}}-\frac{\gamma_{n-1}}{1-\beta_{n-1}}\right|=0$.

Then $\left\{x_{n}\right\}$ converges strongly to $q \in F$, which solves the following VIP:

$$
\langle q-f(q), J(q-p)\rangle \leq 0, \quad \forall p \in F
$$

\section{Three-step explicit iterative algorithm}

In this section, we introduce our three-step explicit iterative algorithm and show strong convergence of the proposed algorithm.

Theorem 6.1 Let $C$ be a nonempty closed convex subset of a uniformly convex Banach space $X$ which has a uniformly Gâteaux differentiable norm. Let $\Pi_{C}$ be a sunny nonexpansive retraction from $X$ onto $C$. Let the mapping $B_{i}: C \rightarrow X$ be $\lambda_{i}$-strictly pseudocontractive and $\alpha_{i}$-strongly accretive with $\alpha_{i}+\lambda_{i} \geq 1$ for $i=1,2$. Let $f: C \rightarrow C$ be a contraction with coefficient $\rho \in(0,1)$. Let $\left\{S_{n}\right\}_{n=0}^{\infty}$ be an infinite family of nonexpansive mappings of $C$ into itself such that $F=\bigcap_{i=0}^{\infty} \operatorname{Fix}\left(S_{i}\right) \cap \Omega \neq \emptyset$, where $\Omega$ is the fixed point set of the mapping $G=\Pi_{C}\left(I-\mu_{1} B_{1}\right) \Pi_{C}\left(I-\mu_{2} B_{2}\right)$. For arbitrarily given $x_{0} \in C$, let $\left\{x_{n}\right\}$ be the sequence generated by

$$
\left\{\begin{array}{l}
z_{n}=\sigma_{n} G\left(x_{n}\right)+\left(1-\sigma_{n}\right) S_{n} G\left(x_{n}\right) \\
y_{n}=\alpha_{n} f\left(x_{n}\right)+\left(1-\alpha_{n}\right) z_{n}, \\
x_{n+1}=\beta_{n} x_{n}+\gamma_{n} y_{n}+\delta_{n} S_{n} G\left(y_{n}\right), \quad \forall n \geq 0,
\end{array}\right.
$$

where $1-\frac{\lambda_{i}}{1+\lambda_{i}}\left(1-\sqrt{\frac{1-\alpha_{i}}{\lambda_{i}}}\right) \leq \mu_{i} \leq 1$ for $i=1,2$. Suppose that $\left\{\sigma_{n}\right\},\left\{\alpha_{n}\right\},\left\{\beta_{n}\right\},\left\{\gamma_{n}\right\}$ and $\left\{\delta_{n}\right\}$ are the sequences in $(0,1)$ satisfying the following conditions:

(i) $0<\liminf _{n \rightarrow \infty} \sigma_{n} \leq \limsup _{n \rightarrow \infty} \sigma_{n}<1$;

(ii) $\lim _{n \rightarrow \infty} \alpha_{n}=0$ and $\sum_{n=0}^{\infty} \alpha_{n}=\infty$;

(iii) $\beta_{n}+\gamma_{n}+\delta_{n}=1, \forall n \geq 0$, and $\liminf _{n \rightarrow \infty} \delta_{n}>0$;

(iv) $0<\liminf _{n \rightarrow \infty} \beta_{n} \leq \limsup _{n \rightarrow \infty}\left(\beta_{n}+\delta_{n}\right)<1$;

(v) $\lim _{n \rightarrow \infty}\left|\sigma_{n}-\sigma_{n-1}\right|=0$ and $\lim _{n \rightarrow \infty}\left|\frac{\gamma_{n}}{1-\beta_{n}}-\frac{\gamma_{n-1}}{1-\beta_{n-1}}\right|=0$.

Assume that $\sum_{n=1}^{\infty} \sup _{x \in D}\left\|S_{n} x-S_{n-1} x\right\|<\infty$ for any bounded subset $D$ of $C$, and let $S$ be a mapping of $C$ into itself defined by $S x=\lim _{n \rightarrow \infty} S_{n} x$ for all $x \in C$ and suppose that $\operatorname{Fix}(S)=$ $\bigcap_{i=0}^{\infty} \operatorname{Fix}\left(S_{i}\right)$. Then $\left\{x_{n}\right\}$ converges strongly to $q \in F$, which solves the following VIP:

$$
\langle q-f(q), J(q-p)\rangle \leq 0, \quad \forall p \in F
$$


Proof Take a fixed $p \in F$ arbitrarily. Then by Lemma 2.11 we know that $p=G(p)$ and $S_{n} p=$ $p$ for all $n \geq 0$. Moreover, by Lemma 2.10 we have

$$
\begin{aligned}
\left\|z_{n}-p\right\| & \leq \sigma_{n}\left\|G\left(x_{n}\right)-p\right\|+\left(1-\sigma_{n}\right)\left\|S_{n} G\left(x_{n}\right)-p\right\| \\
& \leq\left\|x_{n}-p\right\|,
\end{aligned}
$$

and

$$
\begin{aligned}
\left\|y_{n}-p\right\| & \leq \alpha_{n}\left\|f\left(x_{n}\right)-f(p)\right\|+\alpha_{n}\|f(p)-p\|+\left(1-\alpha_{n}\right)\left\|z_{n}-p\right\| \\
& \leq \alpha_{n} \rho\left\|x_{n}-p\right\|+\alpha_{n}\|f(p)-p\|+\left(1-\alpha_{n}\right)\left\|x_{n}-p\right\| \\
& =\left(1-\alpha_{n}(1-\rho)\right)\left\|x_{n}-p\right\|+\alpha_{n}\|f(p)-p\| .
\end{aligned}
$$

From (6.1) and (6.4) we obtain

$$
\begin{aligned}
\left\|x_{n+1}-p\right\| & \leq \beta_{n}\left\|x_{n}-p\right\|+\gamma_{n}\left\|y_{n}-p\right\|+\delta_{n}\left\|S_{n} G\left(y_{n}\right)-p\right\| \\
& \leq \beta_{n}\left\|x_{n}-p\right\|+\left(1-\beta_{n}\right)\left\|y_{n}-p\right\| \\
& \leq \beta_{n}\left\|x_{n}-p\right\|+\left(1-\beta_{n}\right)\left[\left(1-\alpha_{n}(1-\rho)\right)\left\|x_{n}-p\right\|+\alpha_{n}\|f(p)-p\|\right] \\
& =\left[1-\left(1-\beta_{n}\right)(1-\rho) \alpha_{n}\right]\left\|x_{n}-p\right\|+\left(1-\beta_{n}\right)(1-\rho) \alpha_{n} \cdot \frac{\|f(p)-p\|}{1-\rho} \\
& \leq \max \left\{\left\|x_{0}-p\right\|, \frac{\|f(p)-p\|}{1-\rho}\right\},
\end{aligned}
$$

which implies that $\left\{x_{n}\right\}$ is bounded. By Lemma 2.10 we know from (6.3) and (6.4) that $\left\{y_{n}\right\}$, $\left\{z_{n}\right\},\left\{G\left(x_{n}\right)\right\}$ and $\left\{G\left(y_{n}\right)\right\}$ are bounded.

Let us show that $\left\|x_{n+1}-x_{n}\right\| \rightarrow 0$ and $\left\|x_{n}-y_{n}\right\| \rightarrow 0$ as $n \rightarrow \infty$. As a matter of fact, from (6.1), we have

$$
\begin{aligned}
z_{n}-z_{n-1}= & \sigma_{n}\left(G\left(x_{n}\right)-G\left(x_{n-1}\right)\right)+\left(\sigma_{n}-\sigma_{n-1}\right)\left(G\left(x_{n-1}\right)-S_{n-1} G\left(x_{n-1}\right)\right) \\
& +\left(1-\sigma_{n}\right)\left(S_{n} G\left(x_{n}\right)-S_{n-1} G\left(x_{n-1}\right)\right),
\end{aligned}
$$

and

$$
y_{n}-y_{n-1}=\alpha_{n}\left(f\left(x_{n}\right)-f\left(x_{n-1}\right)\right)+\left(\alpha_{n}-\alpha_{n-1}\right)\left(f\left(x_{n-1}\right)-z_{n-1}\right)+\left(1-\alpha_{n}\right)\left(z_{n}-z_{n-1}\right) .
$$

It follows that

$$
\begin{aligned}
\left\|z_{n}-z_{n-1}\right\| \leq & \sigma_{n}\left\|G\left(x_{n}\right)-G\left(x_{n-1}\right)\right\|+\left|\sigma_{n}-\sigma_{n-1}\right|\left\|G\left(x_{n-1}\right)-S_{n-1} G\left(x_{n-1}\right)\right\| \\
& +\left(1-\sigma_{n}\right)\left(\left\|S_{n} G\left(x_{n}\right)-S_{n} G\left(x_{n-1}\right)\right\|+\left\|S_{n} G\left(x_{n-1}\right)-S_{n-1} G\left(x_{n-1}\right)\right\|\right) \\
\leq & \left\|G\left(x_{n}\right)-G\left(x_{n-1}\right)\right\|+\left|\sigma_{n}-\sigma_{n-1}\right|\left\|G\left(x_{n-1}\right)-S_{n-1} G\left(x_{n-1}\right)\right\| \\
& +\left\|S_{n} G\left(x_{n-1}\right)-S_{n-1} G\left(x_{n-1}\right)\right\| \\
\leq & \left\|x_{n}-x_{n-1}\right\|+\left|\sigma_{n}-\sigma_{n-1}\right|\left\|G\left(x_{n-1}\right)-S_{n-1} G\left(x_{n-1}\right)\right\| \\
& +\left\|S_{n} G\left(x_{n-1}\right)-S_{n-1} G\left(x_{n-1}\right)\right\|,
\end{aligned}
$$


and

$$
\begin{aligned}
\left\|y_{n}-y_{n-1}\right\| \leq & \alpha_{n} \rho\left\|x_{n}-x_{n-1}\right\|+\left|\alpha_{n}-\alpha_{n-1}\right|\left\|f\left(x_{n-1}\right)-z_{n-1}\right\| \\
& +\left(1-\alpha_{n}\right)\left[\left\|x_{n}-x_{n-1}\right\|+\left|\sigma_{n}-\sigma_{n-1}\right|\left\|G\left(x_{n-1}\right)-S_{n-1} G\left(x_{n-1}\right)\right\|\right. \\
& \left.+\left\|S_{n} G\left(x_{n-1}\right)-S_{n-1} G\left(x_{n-1}\right)\right\|\right] \\
\leq & \left(1-\alpha_{n}(1-\rho)\right)\left\|x_{n}-x_{n-1}\right\|+\left|\sigma_{n}-\sigma_{n-1}\right|\left\|G\left(x_{n-1}\right)-S_{n-1} G\left(x_{n-1}\right)\right\| \\
& +\left|\alpha_{n}-\alpha_{n-1}\right|\left\|f\left(x_{n-1}\right)-z_{n-1}\right\|+\left\|S_{n} G\left(x_{n-1}\right)-S_{n-1} G\left(x_{n-1}\right)\right\| .
\end{aligned}
$$

Now, we write $x_{n}=\beta_{n-1} x_{n-1}+\left(1-\beta_{n-1}\right) v_{n-1}, \forall n \geq 1$, where $v_{n-1}=\frac{x_{n}-\beta_{n-1} x_{n-1}}{1-\beta_{n-1}}$. It follows that for all $n \geq 1$,

$$
\begin{aligned}
v_{n}-v_{n-1}= & \frac{x_{n+1}-\beta_{n} x_{n}}{1-\beta_{n}}-\frac{x_{n}-\beta_{n-1} x_{n-1}}{1-\beta_{n-1}} \\
= & \frac{\gamma_{n} y_{n}+\delta_{n} S_{n} G\left(y_{n}\right)}{1-\beta_{n}}-\frac{\gamma_{n-1} y_{n-1}+\delta_{n-1} S_{n-1} G\left(y_{n-1}\right)}{1-\beta_{n-1}} \\
= & \frac{\gamma_{n}\left(y_{n}-y_{n-1}\right)+\delta_{n}\left(S_{n} G\left(y_{n}\right)-S_{n-1} G\left(y_{n-1}\right)\right)}{1-\beta_{n}} \\
& +\left(\frac{\gamma_{n}}{1-\beta_{n}}-\frac{\gamma_{n-1}}{1-\beta_{n-1}}\right) y_{n-1}+\left(\frac{\delta_{n}}{1-\beta_{n}}-\frac{\delta_{n-1}}{1-\beta_{n-1}}\right) S_{n-1} G\left(y_{n-1}\right) .
\end{aligned}
$$

This together with (6.5) implies that

$$
\begin{aligned}
& \left\|v_{n}-v_{n-1}\right\| \\
& \leq \frac{\left\|\gamma_{n}\left(y_{n}-y_{n-1}\right)+\delta_{n}\left(S_{n} G\left(y_{n}\right)-S_{n-1} G\left(y_{n-1}\right)\right)\right\|}{1-\beta_{n}}+\left|\frac{\gamma_{n}}{1-\beta_{n}}-\frac{\gamma_{n-1}}{1-\beta_{n-1}}\right|\left\|y_{n-1}\right\| \\
& +\left|\frac{\delta_{n}}{1-\beta_{n}}-\frac{\delta_{n-1}}{1-\beta_{n-1}}\right|\left\|S_{n-1} G\left(y_{n-1}\right)\right\| \\
& \leq \frac{\gamma_{n}\left\|y_{n}-y_{n-1}\right\|+\delta_{n}\left(\left\|S_{n} G\left(y_{n}\right)-S_{n} G\left(y_{n-1}\right)\right\|+\left\|S_{n} G\left(y_{n-1}\right)-S_{n-1} G\left(y_{n-1}\right)\right\|\right)}{1-\beta_{n}} \\
& +\left|\frac{\gamma_{n}}{1-\beta_{n}}-\frac{\gamma_{n-1}}{1-\beta_{n-1}}\right|\left\|y_{n-1}\right\|+\left|\frac{\gamma_{n}}{1-\beta_{n}}-\frac{\gamma_{n-1}}{1-\beta_{n-1}}\right|\left\|S_{n-1} G\left(y_{n-1}\right)\right\| \\
& \leq \frac{\gamma_{n}\left\|y_{n}-y_{n-1}\right\|+\delta_{n}\left\|y_{n}-y_{n-1}\right\|}{\gamma_{n}+\delta_{n}}+\frac{\delta_{n}}{\gamma_{n}+\delta_{n}}\left\|S_{n} G\left(y_{n-1}\right)-S_{n-1} G\left(y_{n-1}\right)\right\| \\
& +\left|\frac{\gamma_{n}}{1-\beta_{n}}-\frac{\gamma_{n-1}}{1-\beta_{n-1}}\right|\left(\left\|y_{n-1}\right\|+\left\|S_{n-1} G\left(y_{n-1}\right)\right\|\right) \\
& \leq\left\|y_{n}-y_{n-1}\right\|+\left\|S_{n} G\left(y_{n-1}\right)-S_{n-1} G\left(y_{n-1}\right)\right\| \\
& +\left|\frac{\gamma_{n}}{1-\beta_{n}}-\frac{\gamma_{n-1}}{1-\beta_{n-1}}\right|\left(\left\|y_{n-1}\right\|+\left\|S_{n-1} G\left(y_{n-1}\right)\right\|\right) \\
& \leq\left(1-\alpha_{n}(1-\rho)\right)\left\|x_{n}-x_{n-1}\right\|+\left|\sigma_{n}-\sigma_{n-1}\right|\left\|G\left(x_{n-1}\right)-S_{n-1} G\left(x_{n-1}\right)\right\| \\
& +\left|\alpha_{n}-\alpha_{n-1}\right|\left\|f\left(x_{n-1}\right)-z_{n-1}\right\|+\left\|S_{n} G\left(x_{n-1}\right)-S_{n-1} G\left(x_{n-1}\right)\right\| \\
& +\left\|S_{n} G\left(y_{n-1}\right)-S_{n-1} G\left(y_{n-1}\right)\right\|+\left|\frac{\gamma_{n}}{1-\beta_{n}}-\frac{\gamma_{n-1}}{1-\beta_{n-1}}\right|\left(\left\|y_{n-1}\right\|+\left\|S_{n-1} G\left(y_{n-1}\right)\right\|\right)
\end{aligned}
$$




$$
\begin{aligned}
\leq & \left\|x_{n}-x_{n-1}\right\|+M\left(\left|\sigma_{n}-\sigma_{n-1}\right|+\left|\alpha_{n}-\alpha_{n-1}\right|+\left|\frac{\gamma_{n}}{1-\beta_{n}}-\frac{\gamma_{n-1}}{1-\beta_{n-1}}\right|\right) \\
& +\left\|S_{n} G\left(x_{n-1}\right)-S_{n-1} G\left(x_{n-1}\right)\right\|+\left\|S_{n} G\left(y_{n-1}\right)-S_{n-1} G\left(y_{n-1}\right)\right\|,
\end{aligned}
$$

where $\sup _{n \geq 0}\left\{\left\|G\left(x_{n}\right)\right\|+\left\|S_{n} G\left(x_{n}\right)\right\|+\left\|f\left(x_{n}\right)\right\|+\left\|z_{n}\right\|+\left\|y_{n}\right\|+\left\|S_{n} y_{n}\right\|\right\} \leq M$ for some $M>0$. Since $\left|\sigma_{n}-\sigma_{n-1}\right| \rightarrow 0, \alpha_{n} \rightarrow 0,\left|\frac{\gamma_{n}}{1-\beta_{n}}-\frac{\gamma_{n-1}}{1-\beta_{n-1}}\right| \rightarrow 0$ and the assumption on $\left\{S_{n}\right\}$, we have

$$
\limsup _{n \rightarrow \infty}\left(\left\|v_{n}-v_{n-1}\right\|-\left\|x_{n}-x_{n-1}\right\|\right) \leq 0 .
$$

Utilizing Lemma 2.3, from condition (iv) we obtain that

$$
\lim _{n \rightarrow \infty}\left\|v_{n}-x_{n}\right\|=0
$$

So, we get

$$
\lim _{n \rightarrow \infty}\left\|x_{n+1}-x_{n}\right\|=\lim _{n \rightarrow \infty}\left(1-\beta_{n}\right)\left\|v_{n}-x_{n}\right\|=0
$$

Also, taking into account the boundedness of $\left\{G\left(x_{n}\right)\right\}$ and $\left\{S_{n} G\left(x_{n}\right)\right\}$, by Lemma 2.5 there exists a continuous strictly increasing function $g_{1}:[0, \infty) \rightarrow[0, \infty), g_{1}(0)=0$ such that for $p \in F$

$$
\begin{aligned}
& \left\|z_{n}-p\right\|^{2} \\
& \quad \leq \sigma_{n}\left\|G\left(x_{n}\right)-p\right\|^{2}+\left(1-\sigma_{n}\right)\left\|S_{n} G\left(x_{n}\right)-p\right\|^{2}-\sigma_{n}\left(1-\sigma_{n}\right) g_{1}\left(\left\|G\left(x_{n}\right)-S_{n} G\left(x_{n}\right)\right\|\right) \\
& \quad \leq\left\|x_{n}-p\right\|^{2}-\sigma_{n}\left(1-\sigma_{n}\right) g_{1}\left(\left\|G\left(x_{n}\right)-S_{n} G\left(x_{n}\right)\right\|\right),
\end{aligned}
$$

and hence

$$
\begin{aligned}
& \left\|y_{n}-p\right\|^{2} \\
& \quad \leq\left\|\left(1-\alpha_{n}\right)\left(z_{n}-p\right)\right\|^{2}+2 \alpha_{n}\left\langle f\left(x_{n}\right)-p, J\left(y_{n}-p\right)\right\rangle \\
& \quad \leq\left\|x_{n}-p\right\|^{2}-\sigma_{n}\left(1-\sigma_{n}\right) g_{1}\left(\left\|G\left(x_{n}\right)-S_{n} G\left(x_{n}\right)\right\|\right)+2 \alpha_{n}\left\|f\left(x_{n}\right)-p\right\|\left\|y_{n}-p\right\| .
\end{aligned}
$$

Since $\left\{x_{n}\right\}$ and $\left\{S_{n} G\left(y_{n}\right)\right\}$ both are bounded, by Lemma 2.5 there exists a continuous strictly increasing function $g_{2}:[0, \infty) \rightarrow[0, \infty), g(0)=0$ such that

$$
\begin{aligned}
& \left\|x_{n+1}-p\right\|^{2} \\
& =\left\|\left(\beta_{n}+\delta_{n}\right)\left(\frac{\beta_{n}}{\beta_{n}+\delta_{n}}\left(x_{n}-p\right)+\frac{\delta_{n}}{\beta_{n}+\delta_{n}}\left(S_{n} G\left(y_{n}\right)-p\right)\right)+\gamma_{n}\left(y_{n}-p\right)\right\|^{2} \\
& \leq\left(\beta_{n}+\delta_{n}\right)\left\|\frac{\beta_{n}}{\beta_{n}+\delta_{n}}\left(x_{n}-p\right)+\frac{\delta_{n}}{\beta_{n}+\delta_{n}}\left(S_{n} G\left(y_{n}\right)-p\right)\right\|^{2}+\gamma_{n}\left\|y_{n}-p\right\|^{2} \\
& \leq\left(\beta_{n}+\delta_{n}\right)\left[\frac{\beta_{n}}{\beta_{n}+\delta_{n}}\left\|x_{n}-p\right\|^{2}+\frac{\delta_{n}}{\beta_{n}+\delta_{n}}\left\|S_{n} G\left(y_{n}\right)-p\right\|\right. \\
& \left.-\frac{\beta_{n} \delta_{n}}{\left(\beta_{n}+\delta_{n}\right)^{2}} g_{2}\left(\left\|x_{n}-S_{n} G\left(y_{n}\right)\right\|\right)\right]+\gamma_{n}\left\|y_{n}-p\right\|^{2}
\end{aligned}
$$




$$
\begin{aligned}
\leq & \beta_{n}\left\|x_{n}-p\right\|^{2}+\delta_{n}\left\|S_{n} G\left(y_{n}\right)-p\right\|^{2}-\frac{\beta_{n} \delta_{n}}{\beta_{n}+\delta_{n}} g_{2}\left(\left\|x_{n}-S_{n} G\left(y_{n}\right)\right\|\right)+\gamma_{n}\left\|y_{n}-p\right\|^{2} \\
\leq & \beta_{n}\left\|x_{n}-p\right\|^{2}+\delta_{n}\left\|y_{n}-p\right\|^{2}-\frac{\beta_{n} \delta_{n}}{\beta_{n}+\delta_{n}} g_{2}\left(\left\|x_{n}-S_{n} G\left(y_{n}\right)\right\|\right)+\gamma_{n}\left\|y_{n}-p\right\|^{2} \\
= & \beta_{n}\left\|x_{n}-p\right\|^{2}+\left(1-\beta_{n}\right)\left\|y_{n}-p\right\|^{2}-\frac{\beta_{n} \delta_{n}}{\beta_{n}+\delta_{n}} g_{2}\left(\left\|x_{n}-S_{n} G\left(y_{n}\right)\right\|\right) \\
\leq & \beta_{n}\left\|x_{n}-p\right\|^{2}+\left(1-\beta_{n}\right)\left[\left\|x_{n}-p\right\|^{2}-\sigma_{n}\left(1-\sigma_{n}\right) g_{1}\left(\left\|G\left(x_{n}\right)-S_{n} G\left(x_{n}\right)\right\|\right)\right. \\
& \left.+2 \alpha_{n}\left\|f\left(x_{n}\right)-p\right\|\left\|y_{n}-p\right\|\right]-\frac{\beta_{n} \delta_{n}}{\beta_{n}+\delta_{n}} g_{2}\left(\left\|x_{n}-S_{n} G\left(y_{n}\right)\right\|\right) \\
\leq & \left\|x_{n}-p\right\|^{2}+2 \alpha_{n}\left\|f\left(x_{n}\right)-p\right\|\left\|y_{n}-p\right\|-\left(1-\beta_{n}\right) \sigma_{n}\left(1-\sigma_{n}\right) g_{1}\left(\left\|G\left(x_{n}\right)-S_{n} G\left(x_{n}\right)\right\|\right) \\
& -\frac{\beta_{n} \delta_{n}}{\beta_{n}+\delta_{n}} g_{2}\left(\left\|x_{n}-S_{n} G\left(y_{n}\right)\right\|\right),
\end{aligned}
$$

which immediately yields

$$
\begin{aligned}
(1- & \left.\beta_{n}\right) \sigma_{n}\left(1-\sigma_{n}\right) g_{1}\left(\left\|G\left(x_{n}\right)-S_{n} G\left(x_{n}\right)\right\|\right)+\beta_{n} \delta_{n} g_{2}\left(\left\|x_{n}-S_{n} G\left(y_{n}\right)\right\|\right) \\
& \leq\left(1-\beta_{n}\right) \sigma_{n}\left(1-\sigma_{n}\right) g_{1}\left(\left\|G\left(x_{n}\right)-S_{n} G\left(x_{n}\right)\right\|\right)+\frac{\beta_{n} \delta_{n}}{\beta_{n}+\delta_{n}} g_{2}\left(\left\|x_{n}-S_{n} G\left(y_{n}\right)\right\|\right) \\
& \leq\left\|x_{n}-p\right\|^{2}-\left\|x_{n+1}-p\right\|^{2}+2 \alpha_{n}\left\|f\left(x_{n}\right)-p\right\|\left\|y_{n}-p\right\| \\
& \leq\left(\left\|x_{n}-p\right\|+\left\|x_{n+1}-p\right\|\right)\left\|x_{n}-x_{n+1}\right\|+2 \alpha_{n}\left\|f\left(x_{n}\right)-p\right\|\left\|y_{n}-p\right\| .
\end{aligned}
$$

Since $\alpha_{n} \rightarrow 0,\left\|x_{n+1}-x_{n}\right\| \rightarrow 0$ and $\liminf _{n \rightarrow \infty} \delta_{n}>0$, we deduce from conditions (i) and (iv) that

$$
\lim _{n \rightarrow \infty} g_{1}\left(\left\|G\left(x_{n}\right)-S_{n} G\left(x_{n}\right)\right\|\right)=0 \quad \text { and } \quad \lim _{n \rightarrow \infty} g_{2}\left(\left\|x_{n}-S_{n} G\left(y_{n}\right)\right\|\right)=0
$$

Utilizing the properties of $g_{1}$ and $g_{2}$, we have

$$
\lim _{n \rightarrow \infty}\left\|G\left(x_{n}\right)-S_{n} G\left(x_{n}\right)\right\|=0 \text { and } \lim _{n \rightarrow \infty}\left\|x_{n}-S_{n} G\left(y_{n}\right)\right\|=0 .
$$

In the meantime, according to condition (iv), we have

$$
\liminf _{n \rightarrow \infty} \gamma_{n}=\liminf _{n \rightarrow \infty}\left(1-\beta_{n}-\delta_{n}\right)=1-\limsup _{n \rightarrow \infty}\left(\beta_{n}+\delta_{n}\right)>0 .
$$

Thus, from (6.8) and (6.10) it follows that

$$
\begin{aligned}
\gamma_{n}\left\|y_{n}-x_{n}\right\| & =\left\|\left(x_{n+1}-x_{n}\right)-\delta_{n}\left(S_{n} G\left(y_{n}\right)-x_{n}\right)\right\| \\
& \leq\left\|x_{n+1}-x_{n}\right\|+\delta_{n}\left\|S_{n} G\left(y_{n}\right)-x_{n}\right\| \rightarrow 0 \quad \text { as } n \rightarrow \infty .
\end{aligned}
$$

That is,

$$
\lim _{n \rightarrow \infty}\left\|y_{n}-x_{n}\right\|=0
$$


Since it follows from (6.1) that

$$
\begin{aligned}
\left\|y_{n}-G\left(x_{n}\right)\right\| & =\left\|\alpha_{n}\left(f\left(x_{n}\right)-G\left(x_{n}\right)\right)+\left(1-\alpha_{n}\right)\left(1-\sigma_{n}\right)\left(S_{n} G\left(x_{n}\right)-G\left(x_{n}\right)\right)\right\| \\
& \leq \alpha_{n}\left\|f\left(x_{n}\right)-G\left(x_{n}\right)\right\|+\left(1-\alpha_{n}\right)\left(1-\sigma_{n}\right)\left\|S_{n} G\left(x_{n}\right)-G\left(x_{n}\right)\right\| \\
& \leq \alpha_{n}\left\|f\left(x_{n}\right)-G\left(x_{n}\right)\right\|+\left\|S_{n} G\left(x_{n}\right)-G\left(x_{n}\right)\right\|,
\end{aligned}
$$

we obtain from (6.10) and $\alpha_{n} \rightarrow 0$ that

$$
\lim _{n \rightarrow \infty}\left\|y_{n}-G\left(x_{n}\right)\right\|=0
$$

which together with (6.11) leads to

$$
\lim _{n \rightarrow \infty}\left\|x_{n}-G\left(x_{n}\right)\right\|=0
$$

By (6.10) and Lemma 2.6, we have

$$
\begin{aligned}
\left\|S G\left(x_{n}\right)-G\left(x_{n}\right)\right\| & \leq\left\|S G\left(x_{n}\right)-S_{n} G\left(x_{n}\right)\right\|+\left\|S_{n} G\left(x_{n}\right)-G\left(x_{n}\right)\right\| \\
& \rightarrow 0 \text { as } n \rightarrow \infty .
\end{aligned}
$$

In terms of (6.13) and (6.14), we have

$$
\begin{aligned}
\left\|x_{n}-S x_{n}\right\| & \leq\left\|x_{n}-G\left(x_{n}\right)\right\|+\left\|G\left(x_{n}\right)-S G\left(x_{n}\right)\right\|+\left\|S G\left(x_{n}\right)-S x_{n}\right\| \\
& \leq 2\left\|x_{n}-G\left(x_{n}\right)\right\|+\left\|G\left(x_{n}\right)-S G\left(x_{n}\right)\right\| \\
& \rightarrow 0 \quad \text { as } n \rightarrow \infty .
\end{aligned}
$$

Define a mapping $W x=(1-\theta) S x+\theta G(x)$, where $G$ is defined by $(1.7)$ and $\theta \in(0,1)$ is a constant. Then by Lemma 2.8 we have that $\operatorname{Fix}(W)=\operatorname{Fix}(S) \cap \operatorname{Fix}(G)=F$. We observe that

$$
\begin{aligned}
\left\|x_{n}-W x_{n}\right\| & =\left\|(1-\theta)\left(x_{n}-S x_{n}\right)+\theta\left(x_{n}-G\left(x_{n}\right)\right)\right\| \\
& \leq(1-\theta)\left\|x_{n}-S x_{n}\right\|+\theta\left\|x_{n}-G\left(x_{n}\right)\right\| .
\end{aligned}
$$

From (6.13) and (6.15), we obtain

$$
\lim _{n \rightarrow \infty}\left\|x_{n}-W x_{n}\right\|=0
$$

Utilizing the arguments similar to those of (3.17) in the proof of Theorem 3.1, we can obtain

$$
\limsup _{n \rightarrow \infty}\left\langle f(q)-q, J\left(x_{n}-q\right)\right\rangle \leq 0
$$

where $q=s-\lim _{t \rightarrow 0} x_{t}$ with $x_{t}$ being the fixed point of the contraction $x \mapsto t f(x)+(1-t) W x$; that is, $x_{t}$ solves the fixed point equation $x_{t}=t f\left(x_{t}\right)+(1-t) W x_{t}$. Noticing that $J$ is norm-toweak* uniformly continuous on bounded subsets of $X$, we deduce from (6.11) and (6.17) 
that

$$
\limsup _{n \rightarrow \infty}\left\langle f(q)-q, J\left(y_{n}-q\right)\right\rangle \leq 0
$$

Finally, let us show that $x_{n} \rightarrow q$ as $n \rightarrow \infty$. We observe that

$$
\begin{aligned}
\| y_{n}- & q \|^{2} \\
= & \alpha_{n}\left\langle f\left(x_{n}\right)-f(q), J\left(y_{n}-q\right)\right\rangle+\alpha_{n}\left\langle f(q)-q, J\left(y_{n}-q\right)\right\rangle \\
& +\left(1-\alpha_{n}\right)\left\langle\sigma_{n}\left(G\left(x_{n}\right)-q\right)+\left(1-\sigma_{n}\right)\left(S_{n} G\left(x_{n}\right)-q\right), J\left(y_{n}-q\right)\right\rangle \\
\leq & \alpha_{n} \rho\left\|x_{n}-q\right\|\left\|y_{n}-q\right\|+\left(1-\alpha_{n}\right)\left\|x_{n}-q\right\|\left\|y_{n}-q\right\|+\alpha_{n}\left\langle f(q)-q, J\left(y_{n}-q\right)\right\rangle \\
= & \left(1-\alpha_{n}(1-\rho)\right)\left\|x_{n}-q\right\|\left\|y_{n}-q\right\|+\alpha_{n}\left\langle f(q)-q, J\left(y_{n}-q\right)\right\rangle \\
\leq & \frac{1-\alpha_{n}(1-\rho)}{2}\left(\left\|x_{n}-q\right\|^{2}+\left\|y_{n}-q\right\|^{2}\right)+\alpha_{n}\left\langle f(q)-q, J\left(y_{n}-q\right)\right\rangle \\
\leq & \frac{1-\alpha_{n}(1-\rho)}{2}\left\|x_{n}-q\right\|^{2}+\frac{1}{2}\left\|y_{n}-q\right\|^{2}+\alpha_{n}\left\langle f(q)-q, J\left(y_{n}-q\right)\right\rangle,
\end{aligned}
$$

which implies that

$$
\left\|y_{n}-q\right\|^{2} \leq\left(1-\alpha_{n}(1-\rho)\right)\left\|x_{n}-q\right\|^{2}+\alpha_{n}(1-\rho) \frac{2\left\langle f(q)-q, J\left(y_{n}-q\right)\right\rangle}{1-\rho} .
$$

Also, by (6.1) and the convexity of $\|\cdot\|^{2}$, we get

$$
\begin{aligned}
\left\|x_{n+1}-q\right\|^{2} & \leq \beta_{n}\left\|x_{n}-q\right\|^{2}+\gamma_{n}\left\|y_{n}-q\right\|^{2}+\delta_{n}\left\|y_{n}-q\right\|^{2} \\
& =\beta_{n}\left\|x_{n}-q\right\|^{2}+\left(1-\beta_{n}\right)\left\|y_{n}-q\right\|^{2} .
\end{aligned}
$$

It follows from (6.19) that

$$
\begin{aligned}
\left\|x_{n+1}-q\right\|^{2} \leq & \left(1-\alpha_{n}\left(1-\beta_{n}\right)(1-\rho)\right)\left\|x_{n}-q\right\|^{2} \\
& +\alpha_{n}\left(1-\beta_{n}\right)(1-\rho) \frac{2\left\langle f(q)-q, J\left(y_{n}-q\right)\right\rangle}{1-\rho} .
\end{aligned}
$$

Applying Lemma 2.1 to (6.20), we conclude that $x_{n} \rightarrow q$ as $n \rightarrow \infty$. This completes the proof.

Corollary 6.1 Let $C$ be a nonempty closed convex subset of a uniformly convex Banach space $X$ which has a uniformly Gâteaux differentiable norm. Let $\Pi_{C}$ be a sunny nonexpansive retraction from $X$ onto $C$. Let the mapping $B_{i}: C \rightarrow X$ be $\lambda_{i}$-strictly pseudocontractive and $\alpha_{i}$-strongly accretive with $\alpha_{i}+\lambda_{i} \geq 1$ for $i=1,2$. Let $f: C \rightarrow C$ be a contraction with coefficient $\rho \in(0,1)$. Let $S$ be a nonexpansive mapping of $C$ into itself such that $F=$ $\operatorname{Fix}(S) \cap \Omega \neq \emptyset$, where $\Omega$ is the fixed point set of the mapping $G=\Pi_{C}\left(I-\mu_{1} B_{1}\right) \Pi_{C}\left(I-\mu_{2} B_{2}\right)$. For arbitrarily given $x_{0} \in C$, let $\left\{x_{n}\right\}$ be the sequence generated by

$$
\left\{\begin{array}{l}
z_{n}=\sigma_{n} G\left(x_{n}\right)+\left(1-\sigma_{n}\right) S G\left(x_{n}\right), \\
y_{n}=\alpha_{n} f\left(x_{n}\right)+\left(1-\alpha_{n}\right) z_{n}, \\
x_{n+1}=\beta_{n} x_{n}+\gamma_{n} y_{n}+\delta_{n} S G\left(y_{n}\right), \quad \forall n \geq 0,
\end{array}\right.
$$


where $1-\frac{\lambda_{i}}{1+\lambda_{i}}\left(1-\sqrt{\frac{1-\alpha_{i}}{\lambda_{i}}}\right) \leq \mu_{i} \leq 1$ for $i=1,2$. Suppose that $\left\{\sigma_{n}\right\},\left\{\alpha_{n}\right\},\left\{\beta_{n}\right\},\left\{\gamma_{n}\right\}$ and $\left\{\delta_{n}\right\}$ are the sequences in $(0,1)$ satisfying the following conditions:

(i) $0<\liminf _{n \rightarrow \infty} \sigma_{n} \leq \limsup _{n \rightarrow \infty} \sigma_{n}<1$;

(ii) $\lim _{n \rightarrow \infty} \alpha_{n}=0$ and $\sum_{n=0}^{\infty} \alpha_{n}=\infty$;

(iii) $\beta_{n}+\gamma_{n}+\delta_{n}=1, \forall n \geq 0$, and $\liminf _{n \rightarrow \infty} \delta_{n}>0$;

(iv) $0<\liminf _{n \rightarrow \infty} \beta_{n} \leq \limsup _{n \rightarrow \infty}\left(\beta_{n}+\delta_{n}\right)<1$;

(v) $\lim _{n \rightarrow \infty}\left|\sigma_{n}-\sigma_{n-1}\right|=0$ and $\lim _{n \rightarrow \infty}\left|\frac{\gamma_{n}}{1-\beta_{n}}-\frac{\gamma_{n-1}}{1-\beta_{n-1}}\right|=0$.

Then $\left\{x_{n}\right\}$ converges strongly to $q \in F$, which solves the following VIP:

$$
\langle q-f(q), J(q-p)\rangle \leq 0, \quad \forall p \in F
$$

\section{Competing interests}

The authors declare that they have no competing interests.

\section{Authors' contributions}

All authors participated in the design of this work and performed equally. All authors read and approved the final manuscript.

\section{Author details}

${ }^{1}$ Department of Mathematics, Shanghai Normal University, and Scientific Computing Key Laboratory of Shanghai Universities, Shanghai, 200234, China. '2Department of Mathematics, King Abdulaziz University, P.O. Box 80203, Jeddah, 21589, Saudi Arabia. ${ }^{3}$ Center for Fundamental Science, Kaohsiung Medical University, Kaohsiung, 807, Taiwan.

\section{Acknowledgements}

This article was partially funded by the Deanship of Scientific Research (DSR), King Abdulaziz University. Therefore, the second, third and forth authors gratefully acknowledge with thanks DSR for financial support. The research of the first author was partially supported by the National Science Foundation of China (11071169), Innovation Program of Shanghai Municipal Education Commission (09ZZ133) and Ph.D. Program Foundation of Ministry of Education of China (20123127110002). The authors thank the referees for their valuable comments and suggestions.

Received: 15 April 2013 Accepted: 5 July 2013 Published: 22 July 2013

\section{References}

1. Takahashi, Y, Hashimoto, K, Kato, M: On sharp uniform convexity, smoothness, and strong type, cotype inequalities. J. Nonlinear Convex Anal. 3, 267-281 (2002)

2. Browder, FE: Convergence theorems for sequences of nonlinear operators in Banach spaces. Math. Z. 100, 201-225 (1967)

3. Zeng, LC, Lee, GM, Wong, NC: Ishikawa iteration with errors for approximating fixed points of strictly pseudocontractive mappings of Browder-Petryshyn type. Taiwan. J. Math. 10, 87-99 (2006)

4. Ceng, LC, Wang, CY, Yao, JC: Strong convergence theorems by a relaxed extragradient method for a general system of variational inequalities. Math. Methods Oper. Res. 67, 375-390 (2008)

5. Verma, RU: On a new system of nonlinear variational inequalities and associated iterative algorithms. Math. Sci. Res. Hot-Line 3(8), 65-68 (1999)

6. Korpelevich, GM: An extragradient method for finding saddle points and for other problems. Èkon. Mat. Metody 12 747-756 (1976)

7. Facchinei, F, Pang, JS: Finite-Dimensional Variational Inequalities and Complementarity Problems, vol. I. Springer, New York (2003)

8. Facchinei, F, Pang, JS: Finite-Dimensional Variational Inequalities and Complementarity Problems, vol. II. Springer, New York (2003)

9. Iusem, AN, Svaiter, BF: A variant of Korpelevich's method for variational inequalities with a new search strategy. Optimization 42, 309-321 (1997)

10. Ceng, LC, Yao, JC: An extragradient-like approximation method for variational inequality problems and fixed point problems. Appl. Math. Comput. 190, 205-215 (2007)

11. Solodov, MV, Svaiter, BF: A new projection method for variational inequality problems. SIAM J. Control Optim. 37 765-776 (1999)

12. Censor, Y, Gibali, A, Reich, S: Two extensions of Korpelevich's extragradient method for solving the variational inequality problem in Euclidean space. Technical report (2010)

13. Lions, JL, Stampacchia, G: Variational inequalities. Commun. Pure Appl. Math. 20, 493-512 (1967)

14. Zeng, LC, Yao, JC: Strong convergence theorem by an extragradient method for fixed point problems and variational inequality problems. Taiwan. J. Math. 10, 1293-1303 (2006)

15. liduka, $\mathrm{H}$, Takahashi, W: Strong convergence theorems for nonexpansive mappings and inverse-strongly monotone mappings. Nonlinear Anal. 61, 341-350 (2005)

16. Nadezhkina, N, Takahashi, W: Weak convergence theorem by an extragradient method for nonexpansive mappings and monotone mappings. J. Optim. Theory Appl. 128, 191-201 (2006) 
17. Ceng, LC, Ansari, QH, Yao, JC: An extragradient method for solving split feasibility and fixed point problems. Comput. Math. Appl. 64(4), 633-642 (2012)

18. Ceng, LC, Teboulle, M, Yao, JC: Weak convergence of an iterative method for pseudomonotone variational inequalities and fixed point problems. J. Optim. Theory Appl. 146, 19-31 (2010)

19. Ceng, LC, Ansari, QH, Yao, JC: Relaxed extragradient methods for finding minimum-norm solutions of the split feasibility problem. Nonlinear Anal. 75(4), 2116-2125 (2012)

20. Ceng, LC, Ansari, QH, Yao, JC: Relaxed extragradient iterative methods for variational inequalities. Appl. Math. Comput. 218, 1112-1123 (2011)

21. Yao, Y, Noor, MA, Chen, R, Liou, YC: Strong convergence of three-step relaxed hybrid steepest-descent methods for variational inequalities. Appl. Math. Comput. 201, 175-183 (2008)

22. Verma, RU: Projection methods, algorithms and a new system of nonlinear variational inequalities. Comput. Math. Appl. 41, 1025-1031 (2001)

23. Ceng, LC, Ansari, QH, Wong, NC, Yao, JC: An extragradient-like approximation method for variational inequalities and fixed point problems. Fixed Point Theory Appl. 2011, Article ID 22 (2011)

24. Ceng, LC, Hadjisavvas, N, Wong, NC: Strong convergence theorem by a hybrid extragradient-like approximation method for variational inequalities and fixed point problems. J. Glob. Optim. 46, 635-646 (2010)

25. Ceng, LC, Guu, SM, Yao, JC: Finding common solutions of a variational inequality, a general system of variational inequalities, and a fixed-point problem via a hybrid extragradient method. Fixed Point Theory Appl. 2011, Article ID 626159 (2011)

26. Yao, Y, Yao, JC: On modified iterative method for nonexpansive mappings and monotone mappings. Appl. Math. Comput. 186, 1551-1558 (2007)

27. Censor, Y, Gibali, A, Reich, S: The subgradient extragradient method for solving variational inequalities in Hilbert space. J. Optim. Theory Appl. 148, 318-335 (2011)

28. Aoyama, $\mathrm{K}$, liduka, $\mathrm{H}$, Takahashi, W: Weak convergence of an iterative sequence for accretive operators in Banach spaces. Fixed Point Theory Appl. 2006, Article ID 35390 (2006)

29. Kamimura, S, Takahashi, W: Weak and strong convergence of solutions to accretive operator inclusions and applications. Set-Valued Anal. 8, 361-374 (2000)

30. Takahashi, W, Toyoda, M: Weak convergence theorems for nonexpansive mappings and monotone mappings. J. Optim. Theory Appl. 118, 417-428 (2003)

31. liduka, $\mathrm{H}$, Takahashi, W, Toyoda, M: Approximation of solutions of variational inequalities for monotone mappings. Panam. Math. J. 14, 49-61 (2004)

32. Xu, HK: Inequalities in Banach spaces with applications. Nonlinear Anal. 16, 1127-1138 (1991)

33. Reich, S: Weak convergence theorems for nonexpansive mappings in Banach spaces. J. Math. Anal. Appl. 67, 274-276 (1979)

34. Xu, HK: Iterative algorithms for nonlinear operators. J. Lond. Math. Soc. 66, 240-256 (2002)

35. Cai, G, Bu, SQ: Convergence analysis for variational inequality problems and fixed point problems in 2-uniformly smooth and uniformly convex Banach spaces. Math. Comput. Model. 55, 538-546 (2012)

36. Ceng, LC, Xu, HK, Yao, JC: Strong convergence of an iterative method with perturbed mappings for nonexpansive and accretive operators. Numer. Funct. Anal. Optim. 29(3-4), 324-345 (2008)

37. Aoyama, K, Kimura, Y, Takahashi, W, Toyoda, M: Approximation of common fixed points of a countable family of nonexpansive mappings in a Banach space. Nonlinear Anal. 67, 2350-2360 (2007)

38. Bruck, RE: Properties of fixed point sets of nonexpansive mappings in Banach spaces. Trans. Am. Math. Soc. 179, 251-262 (1973)

39. Suzuki, T: Strong convergence of Krasnoselskii and Mann's type sequences for one parameter nonexpansive semigroups without Bochner integrals. J. Math. Anal. Appl. 305, 227-239 (2005)

40. Yao, Y, Liou, YC, Kang, SM, Yu, YL: Algorithms with strong convergence for a system of nonlinear variational inequalities in Banach spaces. Nonlinear Anal. 74(17), 6024-6034 (2011)

41. Jung, JS: Iterative approaches to common fixed points of nonexpansive mappings in Banach spaces. J. Math. Anal. Appl. 302, 509-520 (2005)

42. Kamimura, S, Takahashi, W: Strong convergence of a proximal-type algorithm in a Banach space. SIAM J. Optim. 13, 938-945 (2002)

43. Jung, JS: A new iteration method for nonexpansive mappings and monotone mappings in Hilbert spaces. J. Inequal. Appl. 2010, Article ID 251761 (2010)

44. Ceng, LC, Latif, A, Yao, JC: On solutions of system of variational inequalities and fixed point problems in Banach spaces. Fixed Point Theory Appl. 2013, Article ID 176 (2013). doi:10.1186/1687-1812-2013-176

45. Eslamian, M, Latif, A: General split feasibility problems in Hilbert spaces. Abstr. Appl. Anal. 2013, Article ID 805104 (2013)

46. Ceng, LC, Khan, AR, Ansari, QH, Yao, JC: Strong convergence of composite iterative schemes for zeros of $m$-accretive operators in Banach spaces. Nonlinear Anal. 70, 1830-1840 (2009)

47. Khan, AR, Akbar, F: Best simultaneous approximations, asymptotically nonexpansive mappings and variational inequalities in Banach spaces. J. Math. Anal. Appl. 354, 469-477 (2009)

48. Ceng, LC, Khan, AR, Ansari, QH, Yao, JC: Viscosity approximation methods for strongly positive and monotone operators. Fixed Point Theory 10, 35-71 (2009) 